\title{
National Health and Nutrition Examination Survey (NHANES)
}

Anthropometry Procedures Manual 


\section{TABLE OF CONTENTS}

\begin{tabular}{|c|c|c|c|c|}
\hline \multicolumn{4}{|c|}{ Chapter } & $\underline{\text { Page }}$ \\
\hline \multirow[t]{6}{*}{1} & \multicolumn{3}{|c|}{ 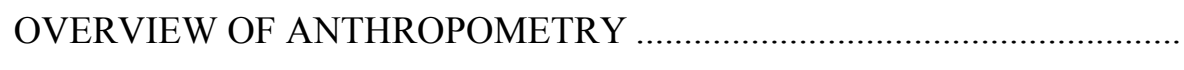 } & $1-1$ \\
\hline & 1.1 & \multicolumn{2}{|c|}{ 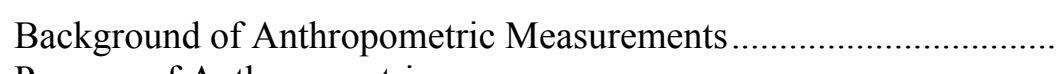 } & $1-1$ \\
\hline & 1.2 & \multicolumn{2}{|c|}{ 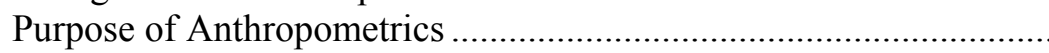 } & $1-1$ \\
\hline & 1.3 & \multicolumn{2}{|c|}{ 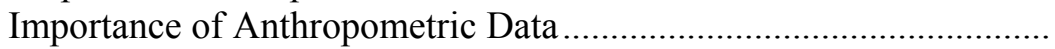 } & $1-2$ \\
\hline & 1.4 & \multicolumn{2}{|c|}{ 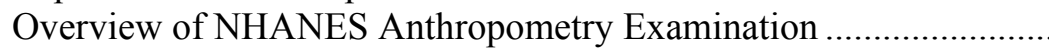 } & $1-4$ \\
\hline & 1.5 & \multicolumn{2}{|c|}{ Role of Anthropometric Examiner and Recorder ................................ } & $1-4$ \\
\hline \multirow[t]{29}{*}{2} & \multicolumn{3}{|c|}{ EQUIPMENT } & $2-1$ \\
\hline & 2.1 & \multirow{2}{*}{\multicolumn{2}{|c|}{$\begin{array}{l}\text { Description of the Body Measurement Room in the MEC } \\
\text { Equipment and Supplies }\end{array}$}} & $2-1$ \\
\hline & \multirow[t]{3}{*}{2.2} & & & $2-1$ \\
\hline & & 2.2 .1 & Equipment. & $2-1$ \\
\hline & & 2.2 .2 & 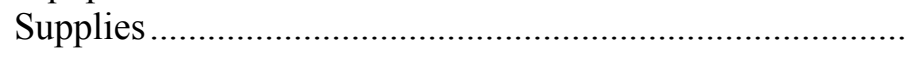 & $2-6$ \\
\hline & \multirow[t]{11}{*}{2.3} & \multicolumn{2}{|c|}{ 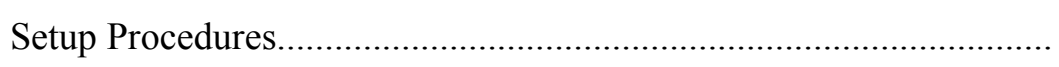 } & $2-9$ \\
\hline & & 2.3 .1 & Calibration Weights . & $2-9$ \\
\hline & & 2.3 .2 & Digital Weight Scale ................. & $2-10$ \\
\hline & & 2.3 .3 & 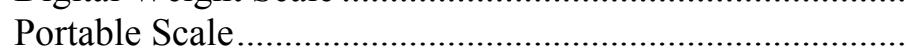 & $2-10$ \\
\hline & & 2.3 .4 & 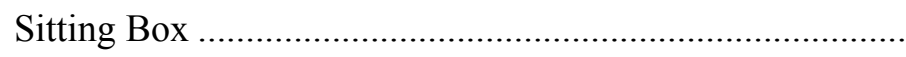 & $2-10$ \\
\hline & & 2.3 .5 & 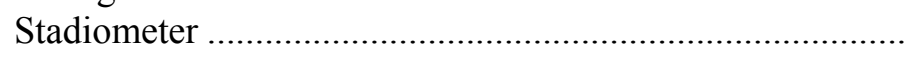 & $2-10$ \\
\hline & & 2.3 .6 & 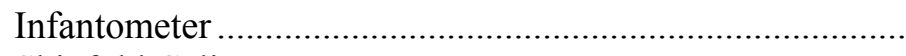 & $2-11$ \\
\hline & & 2.3 .7 & 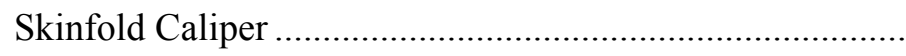 & $2-11$ \\
\hline & & 2.3 .8 & 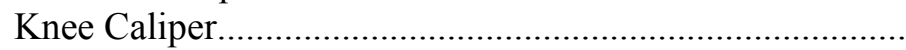 & $2-11$ \\
\hline & & 2.3 .9 & 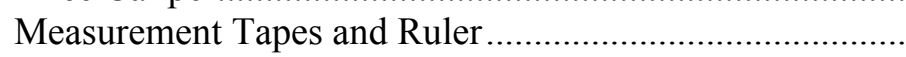 & $2-12$ \\
\hline & & 2.3 .10 & 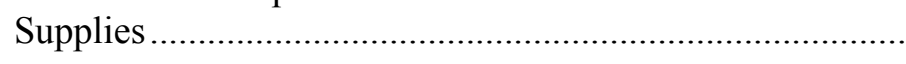 & $2-12$ \\
\hline & 2.4 & \multicolumn{2}{|c|}{ 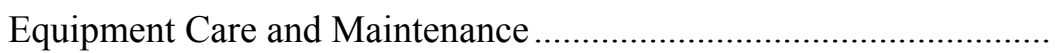 } & $2-12$ \\
\hline & 2.5 & \multirow{2}{*}{\multicolumn{2}{|c|}{ 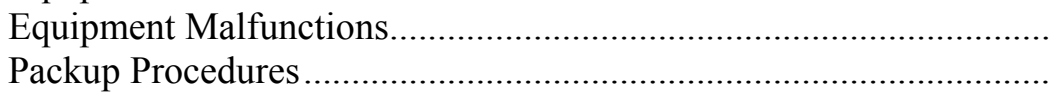 }} & $2-13$ \\
\hline & \multirow[t]{11}{*}{2.6} & & & $2-14$ \\
\hline & & 2.6 .1 & Calibration Weights .... & $2-14$ \\
\hline & & 2.6 .2 & 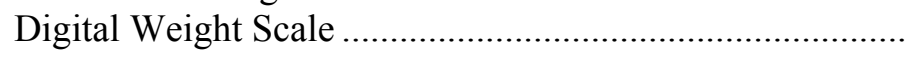 & $2-14$ \\
\hline & & 2.6 .3 & Portable Scale & $2-15$ \\
\hline & & 2.6 .4 & 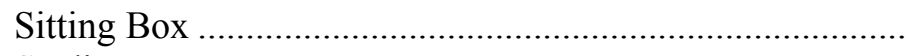 & $2-15$ \\
\hline & & 2.6 .5 & Stadiometer & $2-15$ \\
\hline & & 2.6 .6 & Infantometer & $2-15$ \\
\hline & & 2.6 .7 & 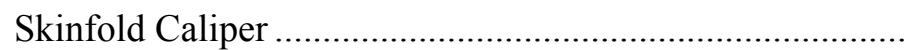 & $2-16$ \\
\hline & & 2.6 .8 & 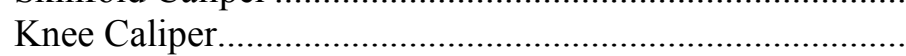 & $2-16$ \\
\hline & & 2.6 .9 & 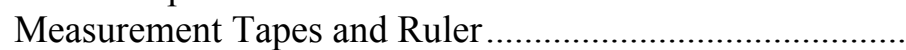 & $2-16$ \\
\hline & & 2.6 .10 & 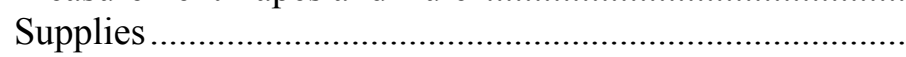 & $2-16$ \\
\hline
\end{tabular}


TABLE OF CONTENTS (continued)

\begin{tabular}{|c|c|c|c|c|}
\hline hant & & & & $\underline{\text { Page }}$ \\
\hline 3 & $\mathrm{EX}$ & INATl & J PROTOCOL .................... & $3-1$ \\
\hline & 3.1 & Eligit & 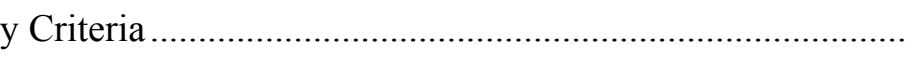 & $3-1$ \\
\hline & 3.2 & Pre-e & nination Procedures & $3-1$ \\
\hline & 3.3 & Gene & 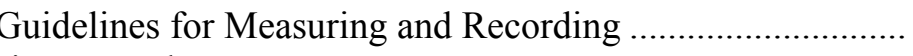 & $3-2$ \\
\hline & 3.4 & Exam & 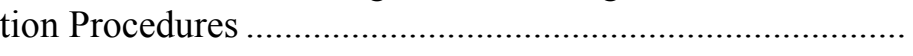 & $3-3$ \\
\hline & & 3.4 .1 & Weight & $3-3$ \\
\hline & & 3.4 .2 & 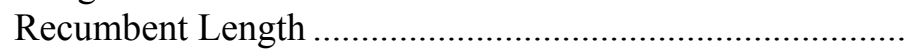 & $3-5$ \\
\hline & & 3.4 .3 & 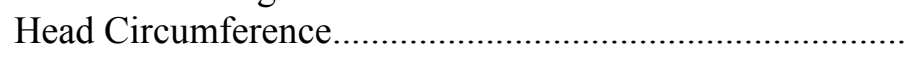 & $3-6$ \\
\hline & & 3.4 .4 & 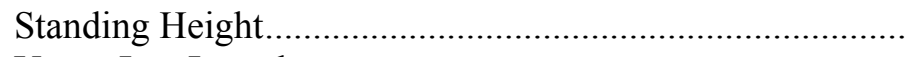 & $3-7$ \\
\hline & & 3.4 .5 & 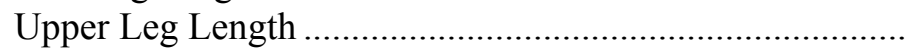 & $3-10$ \\
\hline & & 3.4 .6 & 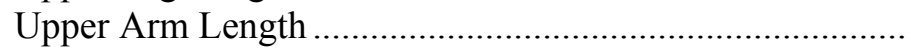 & $3-12$ \\
\hline & & 3.4 .7 & 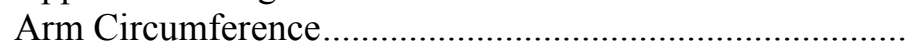 & $3-15$ \\
\hline & & 3.4 .8 & 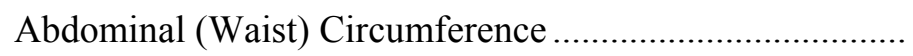 & $3-15$ \\
\hline & & 3.4 .9 & 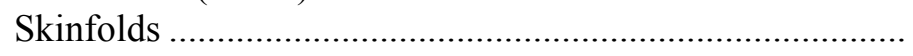 & $3-17$ \\
\hline & 3.5 & Speci & Considerations....... & $3-23$ \\
\hline & & 3.5 .1 & SPs in Street Clothes. & $3-23$ \\
\hline & & 3.5 .2 & 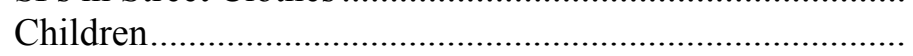 & $3-24$ \\
\hline & & 3.5 .3 & 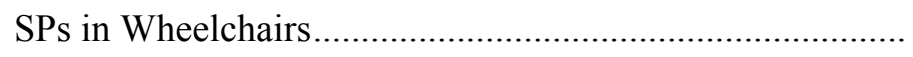 & $3-24$ \\
\hline & & 3.5 .4 & Amputees & $3-25$ \\
\hline & & 3.5.5 & Comprehension or Language Difficulties ............................... & $3-25$ \\
\hline & 3.6 & Post-c & mination Procedures & $3-26$ \\
\hline 4 & ISIS & ATA E & RY ..... & $4-1$ \\
\hline & 4.1 & Gene & Screen Information..... & $4-1$ \\
\hline & 4.2 & Weig & Screen & $4-3$ \\
\hline & & 4.2 .1 & Weight Comments..... & $4-5$ \\
\hline & & 4.2 .2 & 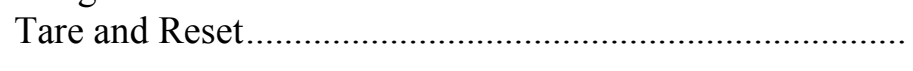 & $4-6$ \\
\hline & 4.3 & Statur & creen ... & $4-7$ \\
\hline & & 4.3.1 & Height Correction..... & $4-10$ \\
\hline & & 4.3.2 & 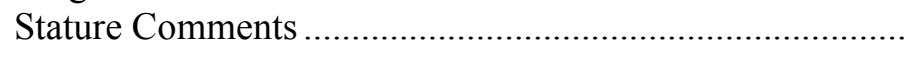 & $4-10$ \\
\hline & 4.4 & Sittin & 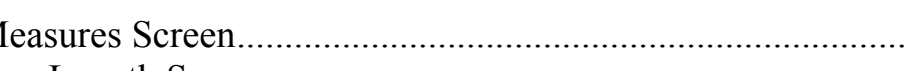 & $4-13$ \\
\hline & 4.5 & Uppe & rm Length Screen.... & \\
\hline
\end{tabular}




\section{TABLE OF CONTENTS (continued)}

Chapter $\quad \underline{\text { Page }}$

4.6 Circumference Measures Screen …................................................ 4-15

4.7 Skinfold Measures Screen ............................................................ 4-16

4.8 SP Information Screen .................................................................

4.9 Amputations Screen ................................................................. 4-18

4.10 Anthropometry Component Status Screen....................................... 4-20

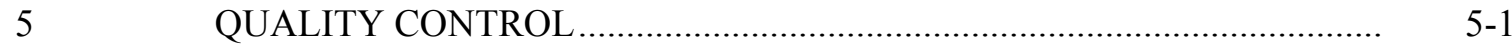

5.1 Introduction to Quality Control for Anthropometry ...........................

5.2 Equipment Calibrations ...................................................................... 5-1

5.2.1 Digital Weight Scale ..................................................... 5-4

5.2.2 Portable Scales ............................................................ $\quad$ 5-9

5.2.3 Stadiometer ..................................................... $\quad 5-10$

5.2.4 Infantometer .....................................................

5.2.5 Skinfold Calipers.......................................................... $\quad 5-14$

5.2.6 Equipment Swap .................................................... $\quad 5-15$

5.3 ISIS Examination Screens ..............................................................

5.4 Observations, Replication, and Review ........................................ $\quad 5-17$

6

SAFETY PROCEDURES …..........................................................

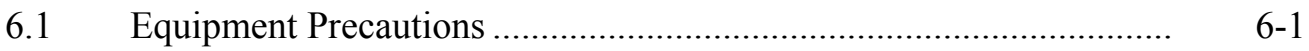

6.2 SP Movement and Positioning........................................................... 6-1

6.3 Emergency Procedures ...................................................................

List of Appendixes

$\underline{\text { Appendix }}$

A Body Measures Recording Form …...................................................... A-1

B Procedures for Conducting Anthropometry Gold Standard Replicate

Examinations

B-1 


\title{
TABLE OF CONTENTS (continued)
}

\author{
List of Tables
}

$\underline{\text { Table }}$

$\underline{\text { Page }}$

3-1 Body measurements by age category

5-1 Anthropometry equipment calibrations

\section{List of Exhibits}

$\underline{\text { Exhibit }}$

Digital weight scale-covered. 


\title{
TABLE OF CONTENTS (continued)
}

\author{
List of Exhibits (continued)
}

\begin{tabular}{|c|c|}
\hline xhibit & \\
\hline $3-4$ & 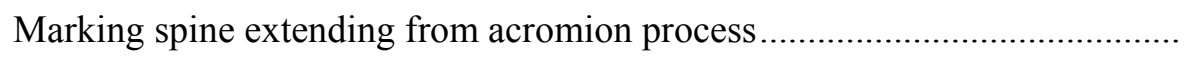 \\
\hline $3-5$ & Correct tape placement for upper arm length............ \\
\hline $3-6$ & Incorrect tape placement for upper arm length \\
\hline $3-7$ & 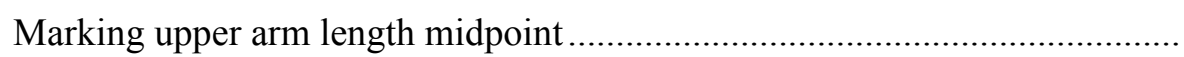 \\
\hline $3-8$ & 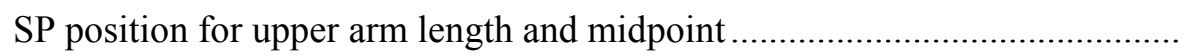 \\
\hline $3-9$ & 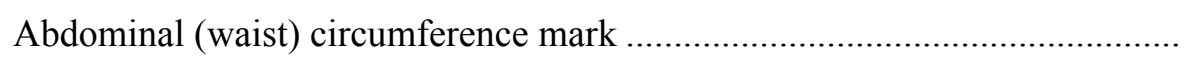 \\
\hline $3-10$ & Measuring tape position for abdominal (waist) circumference.... \\
\hline $3-11$ & 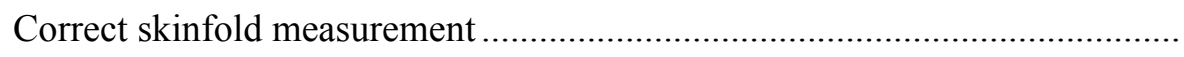 \\
\hline $3-12$ & 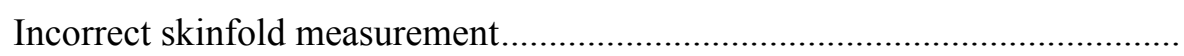 \\
\hline $3-13$ & Triceps skinfold measurement.... \\
\hline $3-14$ & Location of subscapular skinfold \\
\hline $3-15$ & Subscapular skinfold mark.................... \\
\hline $3-16$ & Subscapular skinfold measurement... \\
\hline $4-1$ & Logon SP screen \\
\hline $4-2$ & ISIS screen characteristics .... \\
\hline 4-3 & Edit check box ...... \\
\hline $4-4$ & Weight screen \\
\hline $4-5$ & Weight screen $-\mathrm{lb} \mathrm{kg}$ warning message......... \\
\hline $4-6$ & 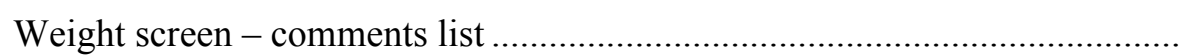 \\
\hline $4-7 \mathrm{a}$ & Stature screen - SPs 0-6 mos.. \\
\hline
\end{tabular}




\title{
TABLE OF CONTENTS (continued)
}

\author{
List of Exhibits (continued)
}

\begin{tabular}{|c|c|c|}
\hline xhibit & & $\underline{\text { Page }}$ \\
\hline $4-7 b$ & 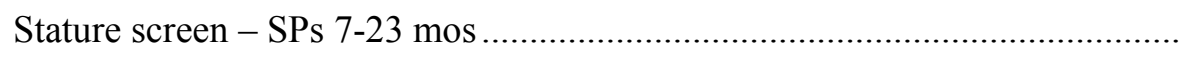 & $4-8$ \\
\hline $4-7 \mathrm{c}$ & 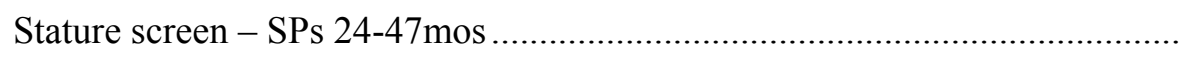 & $4-9$ \\
\hline $4-7 d$ & 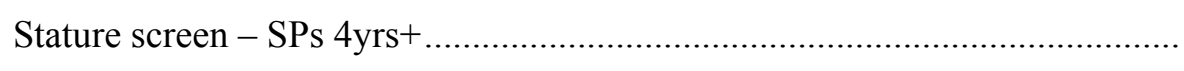 & $4-9$ \\
\hline $4-8 \mathrm{a}$ & 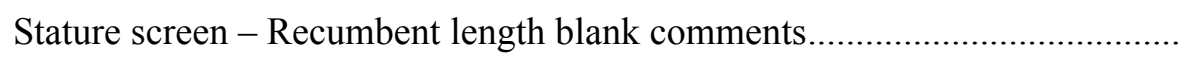 & $4-11$ \\
\hline $4-8 b$ & 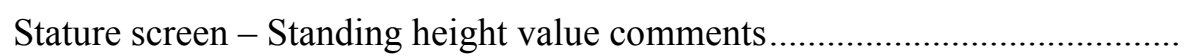 & $4-12$ \\
\hline $4-9$ & Sitting measures screen & $4-13$ \\
\hline $4-10$ & Upper arm length screen ....... & $4-14$ \\
\hline $4-11$ & Circumference measures screen............... & $4-15$ \\
\hline $4-12$ & 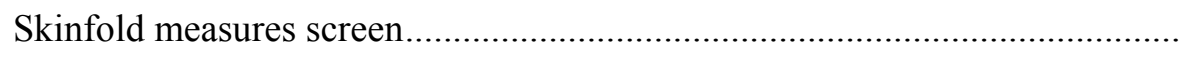 & $4-16$ \\
\hline $4-13$ & SP information screen ................. & $4-17$ \\
\hline $4-14$ & Amputations screen...................... & $4-19$ \\
\hline $4-15$ & 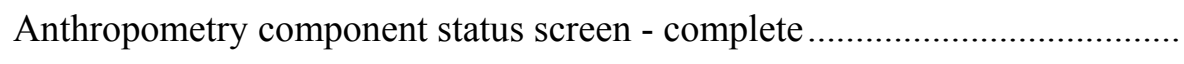 & $4-21$ \\
\hline $4-16$ & 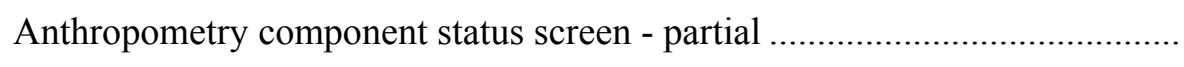 & $4-21$ \\
\hline $5-1$ & Body measures quality control checks..................... & $5-2$ \\
\hline $5-2$ & 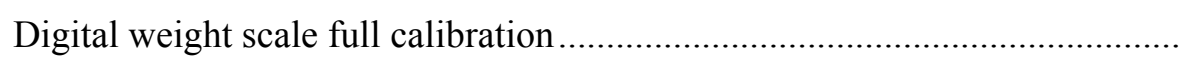 & $5-4$ \\
\hline $5-3$ & Digital weight scale daily calibration .......................... & $5-5$ \\
\hline $5-4$ & Digital scale display device .................... & $5-6$ \\
\hline $5-5$ & 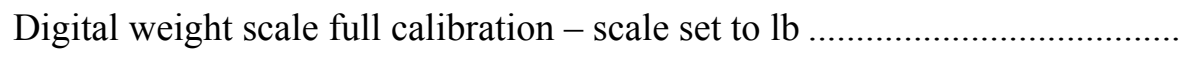 & $5-7$ \\
\hline $5-6$ & 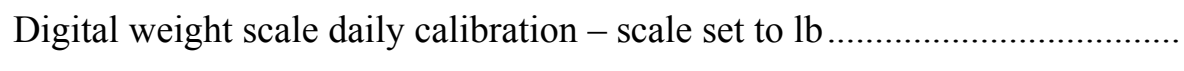 & $5-7$ \\
\hline $5-7$ & 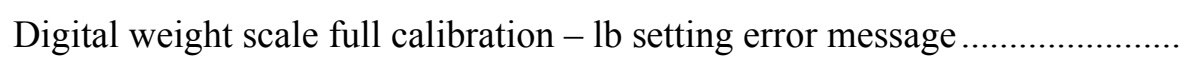 & $5-8$ \\
\hline
\end{tabular}




\title{
TABLE OF CONTENTS (continued)
}

\author{
List of Exhibits (continued)
}

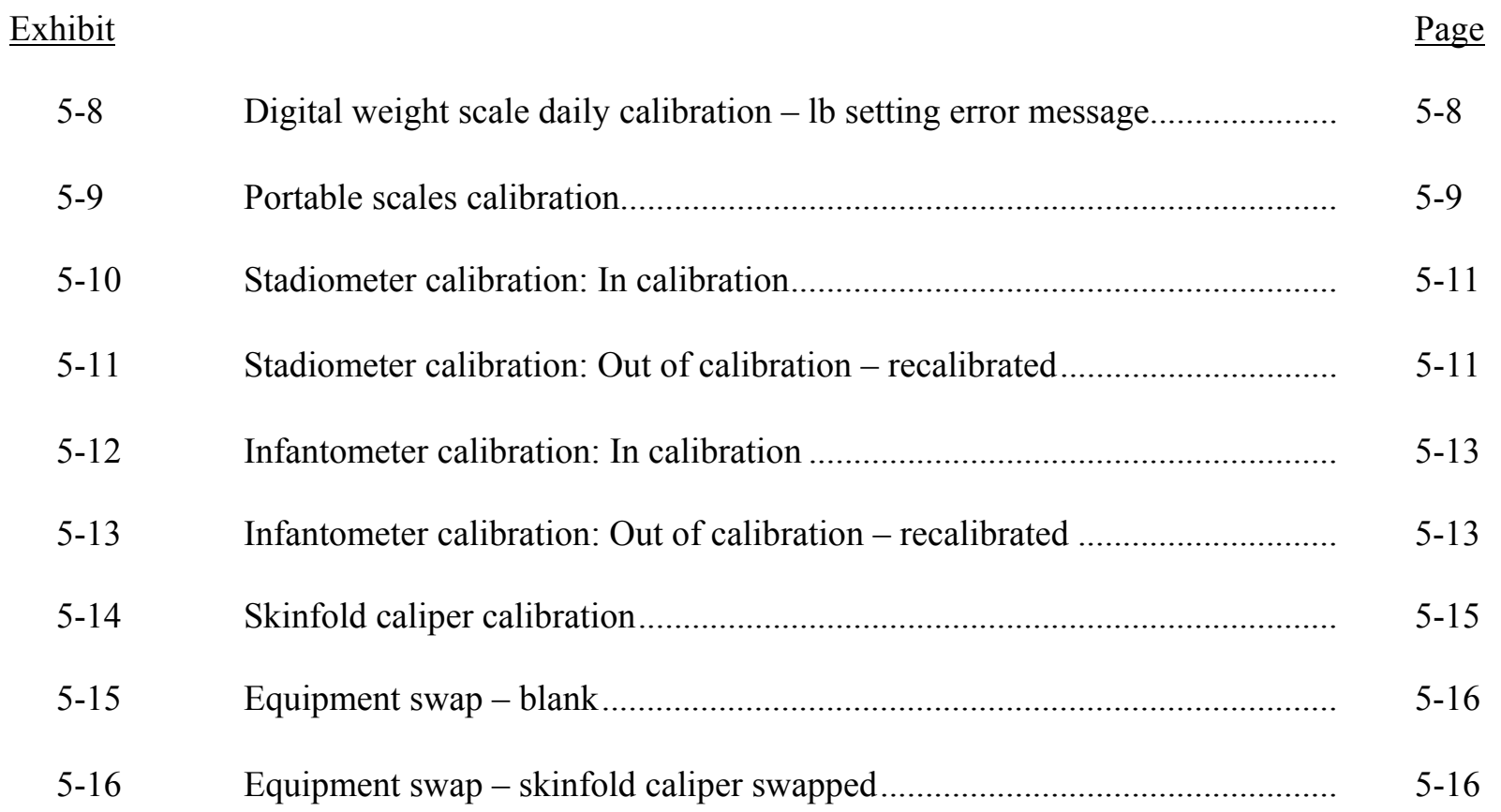




\section{OVERVIEW OF ANTHROPOMETRY}

\subsection{Background of Anthropometric Measurements}

Anthropometry is the study of the measurement of the human body in terms of the dimensions of bone, muscle, and adipose (fat) tissue. The word "anthropometry" is derived from the Greek word "anthropo" meaning "human" and the Greek word "metron" meaning "measure" (Ulajaszek, 1994). The field of anthropometry encompasses a variety of human body measurements. Weight, stature (standing height), recumbent length, skinfold thicknesses, circumferences (head, waist, limb, etc.), limb lengths, and breadths (shoulder, wrist, etc.) are examples of anthropometric measures.

Several indexes and ratios can be derived from anthropometric measurements. Perhaps the most well-known indicator of body fatness is the body mass index or "BMI." BMI values are calculated for NHANES participants using measured height and weight values as follows: weight (kilograms)/height $\left(\right.$ meters $^{2}$ ). BMI criteria are used to screen for weight categories: underweight (BMI values $<18.5$ ), normal or desirable weight (BMI values 18.5-24.9), overweight (BMI values 25.0-29.9), obese-Class I (BMI values 30.0-34.9), obese-Class II (BMI values 35.0-39.9) and extremely obese (BMI values $\geq 40.0$ ) (National Institutes of Health, 1998). NHANES BMI results are used to track weight trends in the U.S. population. The National Institutes of Health, Centers for Disease Control and Prevention (CDC), and many other research groups have reported on the health risks associated with overweight and obesity using NHANES interview and health examination data.

\subsection{Purpose of Anthropometrics}

The purpose of the NHANES anthropometry component is to collect high quality body measurement data using standardized examination procedures and calibrated equipment. Accurate data are fundamental to the evaluation of anthropometric trends over time. It is crucial for researchers to know that the differences that occur between NHANES surveys reflect true differences in the NHANES body measurement values rather than technician and protocol variability and/or measurement error. In order to ensure the collection of high quality data, NHANES staff are trained to follow standardized examination protocols, to calibrate equipment according to a prescribed schedule and method, and to measure and record the survey data with precision. Additionally, retraining sessions, gold standard examinations 
performed by expert examiners, and field observations are conducted on a regular basis to reinforce the importance of measurement precision and standardized data collection methodology.

\section{Importance of Anthropometric Data}

Anthropometry is a key component of nutrition status assessment in children and adults (Simko). The NHANES anthropometry data have been used to track growth and weight trends in the U.S. population for more than thirty years (Flegal, 2002; Hedley, 2004). The anthropometric data for infants and children reflect general health status and dietary adequacy and are used to track trends in growth and development over time. The CDC has used NHANES data to produce national reference standards or "growth charts." The CDC growth charts are used extensively by pediatricians and researchers in the U.S. and abroad (Kuczmarski, 2002). A description of the history and statistical methodology that was used to develop the latest growth charts can be found at the CDC web site: http:/www.cdc.gov/growthcharts/. The highly publicized NHANES data trends showing increases in overweight and obesity among U.S. children and adolescents underscore the importance of NHANES to provide continuous health data (Ogden, 2002).

The NHANES anthropometric data are used to evaluate health and dietary status, disease risk, and body composition changes that occur over the adult lifespan. Researchers in diverse health disciplines including cardiovascular health, gerontology, nutrition, and occupational health use anthropometric data to examine health status and health care utilization trends in U.S. adults. Current U.S. demographic trends project significant growth among race/ethnic subgroups and adults 75 years and older. These trends are reflected in the NHANES sample, which includes a large proportion of adults 60 years and older, African Americans, and Hispanics. Racial and ethnic minorities are more likely to be medically underserved compared to non-Hispanic whites in terms of having a regular source of health care, health insurance, and access to preventive health services. Valuable health information on population groups that might not be reflected in other health reporting systems is routinely collected in surveys sponsored by the National Center for Health Statistics (NCHS).

With respect to the "graying" U.S. population, the "baby boomer" generation is approaching retirement age. What will their quality of life be like during their "golden years?" How much health care will older persons need to maintain their health? What types of health conditions will be more prevalent among older Americans? Functional status and general well-being are inevitably linked to weight status. 
A recent analysis of the NHANES 1999-2002 data using BMI criteria determined that overweight and obese individuals were more likely to report fair or poor health (rather than good or excellent health), activity limitations, and more health provider visits per year compared to normal weight adults (McDowell, 2006).

The NHANES skinfold thickness and circumference measures assess subcutaneous and visceral fat tissue. Recent reports based on NHANES showed increasing mid-arm circumference measurements in children and adults (Ostchega, et al. 2005 and 2006). These data have been used to formulate guidelines for health practitioners regarding the selection of blood pressure cuff sizes for children and adults. In addition, the NHANES waist circumference data are widely used to assess cardiovascular disease risk. Specifically, individuals who have large deposits of abdominal fat tissue are at increased risk for hypertension, adult-onset diabetes mellitus, cardiovascular disease, gallstones, arthritis, and some forms of cancer.

Although much of the attention in today's popular media is on overweight and obesity, NHANES data can also be used to track trends in underweight. Underweight may occur due to a variety of factors, such as: poor nutrition and eating habits, substance abuse, chronic illness, medication therapy, surgical procedures, and other health problems.

Finally, NHANES is the only study that produces national estimates for the U.S. population-all ages and all sizes. NCHS continues to publish reports based on the NHANES anthropometry data. National reference data for children and adults based on NHANES 1999-2002 are available through the CDC web site at the following link: http://www.cdc.gov/nchs/data/ad/ad361.pdf . The NHANES body measures examination data files are also publicly available and may be downloaded from the NHANES web site: http://www.cdc.gov/nchs/nhanes.htm. To retrieve information on the NHANES anthropometry training methodologies and examination protocols, refer to the following link: http://www.cdc.gov/nchs/about/major/nhanes/datalink.htm. This offers access to the NHANES anthropometric procedures training manuals used for each NHANES, as well as the NHANES III Anthropometric Procedures Video. Lastly, hyperlinks for data, questionnaires, and manuals for each NHANES survey are also provided on the NHANES web site. 


\subsection{Overview of NHANES Anthropometry Examination}

The NHANES anthropometry or body measures examination is conducted in trailer 1 of the mobile examination center (MEC). All NHANES participants or sample persons (SPs) are eligible for the anthropometry examination component. Specific measurements are completed dependent on the age of the participant. The complete set of measurements includes weight, height, upper leg and arm length, mid-arm and waist circumference, and triceps and subscapular skinfolds. The anthropometry examination protocol and procedures are described in Chapter 3, Examination Protocol. A health technologist and recorder work as a team to collect the anthropometry data. Examination results are saved to the study database using the Integrated Survey Information System (ISIS) anthropometry computer application. Funding for the anthropometry examination component is provided solely by NCHS.

\subsection{Role of Anthropometric Examiner and Recorder}

The anthropometry component is staffed by trained health technicians and recorders. The NHANES health technologists may serve as examiners or recorders for this component. In addition, other MEC staff are trained to record anthropometry examination results. When two health technologists are assigned by the MEC coordinator to the anthropometry room, the technologist assigned as the examiner should complete the entire examination.

The examiner will position the participant, take all measurements, and tell the recorder the measurement values to record as described in Chapter 3, Examination Protocol. The recorder will enter the examination results in the ISIS anthropometry component software application. The ISIS module is programmed to present the anthropometry data entry screens in the correct sequence based on the age of the participant. The ISIS interface is also pre-programmed with age- and gender-specific edit ranges for each measurement, based on results from earlier NHANES. This feature is designed to reduce data entry errors that may result from either incorrect measurement or data entry errors. See Section 4.3, ISIS Examination Screens, for details on the quality control features of the ISIS anthropometry application. If a recorded value falls outside the pre-programmed edit range, the ISIS system will alert the recorder that the recorded value is unusual. The recorder should always ask the examiner to verify the measurement value before proceeding to the next measurement. If a measurement or recording error was made, the recorder will enter the correct value; if the original value is correct, the value is retained. Since NHANES includes participants of all shapes, sizes, and body builds, some unusual values are legitimate; however, 
measurement and recording errors do occasionally occur. Therefore teamwork between the examiner and recorder is essential in order to measure and record accurate data.

Another important duty of the recorder is to help the examiner position the participants during their examination. In this role the recorder also alerts the examiner if the respondent needs to be repositioned. For example, when the waist circumference is determined, the recorder checks that the tape measure lies parallel to the floor and snug, but without compressing the skin. Also, the recorder participates in taking recumbent length measurements on infants and young children. Assistance is needed because several body sites must be aligned to obtain an accurate measurement. Lastly, the recorder marks body sites that are measured by the examiner and hands equipment and supplies to the examiner when needed.

\section{References}

Anthropometric reference data for children and adults: U.S. population. 1999-2002. Advance data from vital and health statistics; no. 361. Hyattsville, MD: National Center for Health Statistics. 2005. Available at: http://www.cdc.gov/nchs/data/ad/ad361.pdf

Clinical Guidelines on the Identification, Evaluation, and Treatment of Overweight and Obesity in Adults--The Evidence Report. National Institutes of Health. Obes Res. 1998, Sep. 6. Suppl 2:51S209S. Also available at: http://www.nhlbi.nih.gov/guidelines/obesity/ob gdlns.htm (Accessed August 21, 2006).

Flegal KM, Carroll MD, Ogden CL, Johnson CL. Prevalence and trends in obesity among U.S. adults, 1999-2000. JAMA. 2002, Oct. 9. 288(14):1723-7.

Hedley AA, Ogden CL, Johnson CL, Carroll MD, Curtin LR, Flegal KM. Prevalence of overweight and obesity among U.S. children, adolescents, and adults, 1999-2002. JAMA. 2004, June 16. 291(23):2847-50.

Kuczmarski RJ, Ogden CL, Guo SS, Grummer-Strawn LM, Flegal KM, Mei Z, Wei R, Curtin LR, Roche AF, Johnson CL. 2000 CDC Growth Charts for the United States: methods and development. (2002 May). Vital Health Stat 11. (246):1-190.

McDowell MA, Hughes JP, Borrud LG. Health characteristics of U.S. adults by body mass index category: results from NHANES 1999-2002. (2006). Public Health Rep., 121:67-73.

Ogden CL, Flegal KM, Carroll MD, Johnson CL. Prevalence and trends in overweight among U.S. children and adolescents, 1999-2000. 2002, Oct 9. JAMA. 288(14):1728-32. 
Ostchega, Y., et al. Tables for the selection of correct blood pressure cuff size based on self-reported height and weight and estimating equations for mid-arm circumference: data from the U.S. National Health and Nutrition Examination Survey. (2006) Jan. J Hum Hypertens. 20(1):15-22.

Ostchega, Y., et al. U.S. demographic trends in mid-arm circumference and recommended blood pressure cuffs: 1988-2002. 2005 Nov. J Hum Hypertens. 19(11):885-91.

Simko M and Cowell C. (1995). Nutrition Assessment: A Comprehensive Guide for Planning Intervention. Second Edition. Jones and Bartlett.

Ulijaszek, SJ. (1994). Anthropology: The Individual and the Population. Cambridge University Press. 


\section{EQUIPMENT}

\subsection{Description of the Body Measurement Room in the MEC}

The body measurement room is located in trailer 1 of the MEC. The room is equipped with unique features designed to facilitate accurate and efficient measurement, including: a wall mirror to facilitate waist circumference measurements; a custom-built box where SPs can sit for the upper leg length measurement; and a digital floor scale that is housed in an opening in the floor. Additionally, the electronic equipment, namely the scale, stadiometer, and infantometer are linked electronically to the NHANES Integrated Survey Information System (ISIS) for quick and accurate data entry.

\subsection{Equipment and Supplies}

A brief description of the anthropometry component equipment and supplies is provided below. In addition, Exhibits 2-1 to 2-13 contain photographs of these items for reference.

\subsubsection{Equipment}

- Calibration weights:

- Digital weight scale:

- Portable scales:
Each MEC has a set of fifteen 10-kilogram weights. The weights are used to calibrate the portable scales at the beginning of a stand; and the digital weight scale daily and at the beginning, middle, and end of a stand.

The digital weight scale used to weigh SPs is built into the exam room floor. This high performance instrument is linked directly to the ISIS anthropometry application. The maximum capacity of the digital weight scale is 440 pounds.

Two portable scales are available on each MEC for use in the event of a malfunction of the digital weight scale, or if a SP's weight exceeds the digital scale capacity. Each scale has a maximum capacity of 440 pounds. Four AA batteries are used to power each scale. 
- Sitting box:

- Stadiometer:

- Calibration rod:

- Infantometer:

- Skinfold calipers:

- Step wedge standard:

- Knee caliper:

- Head circumference tape:

- Steel measuring tape:
A white rectangular box is used as a seat for the SP during the upper leg length measurement. Also, some children may need to stand on the box while the health technologist takes their body measurements.

The stadiometer is used to measure stature in SPs aged 2 years and older. A digital measurement device is connected to the acrylic head piece and interfaces directly with the ISIS anthropometry application.

An 80 centimeter metal rod is used to calibrate the stadiometer at the beginning of a stand and on a weekly basis. When not in use, the rod is stored in the holding grips on the side of the stadiometer.

The infantometer is used to measure the recumbent length of SPs from birth through age 47 months. A digital measurement device is connected to the acrylic foot piece and interfaces directly with the ISIS anthropometry application.

A skinfold caliper is used to take subscapular and triceps skinfold measurements. Two skinfold calipers are available on each MEC. When not in use, each skinfold caliper is stored in a protective case in the cabinet drawer.

Readings taken from a metal, pyramid step wedge are used to calibrate the skinfold calipers at the beginning of a stand and on a weekly basis.

A metal, sliding caliper is used to mark the distal end of the femur in the determination of the upper leg length. Two knee calipers are available on each MEC. When not in use, the caliper is stored in a protective case in the cabinet drawer.

Maximal head circumference of SPs from birth through age 6 months is measured using a plastic measuring tape. The measurement end is inserted into a slot at the zero end of the tape.

A retractable steel measuring tape is used to take length and circumference measurements. 
- Height adjustment ruler:

A 15 centimeter plastic ruler is used to correct stature measurements when SPs have hair styles that interfere with the stadiometer headpiece placement; or when they are unwilling to remove their shoes. The recorder enters the height correction factor in ISIS, which automatically calculates an adjusted height value. 
Exhibit 2-1. Calibration weight (10-kg)

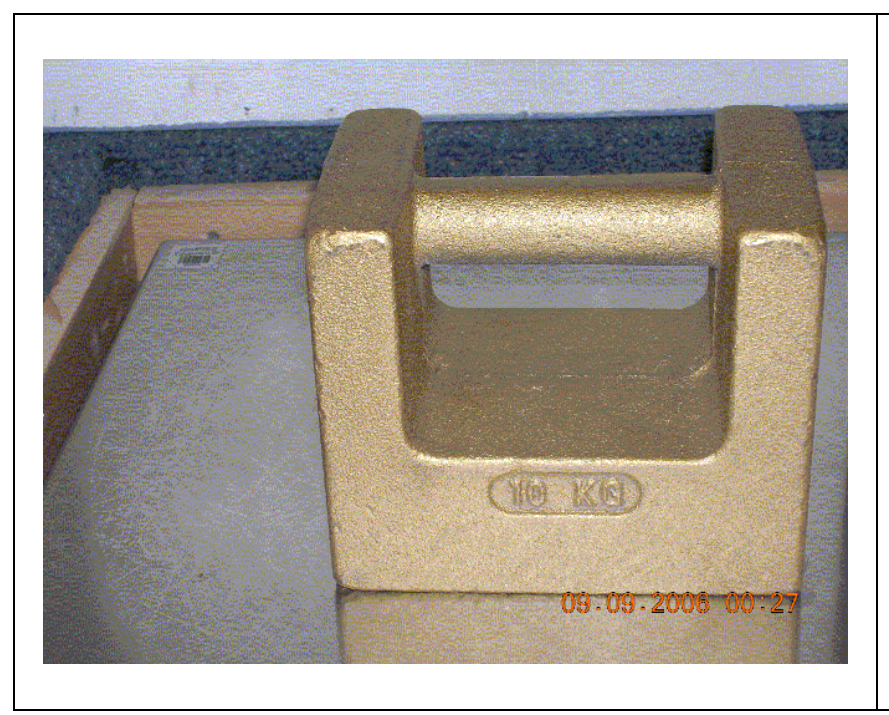

Exhibit 2-3. Digital weight scale-uncovered

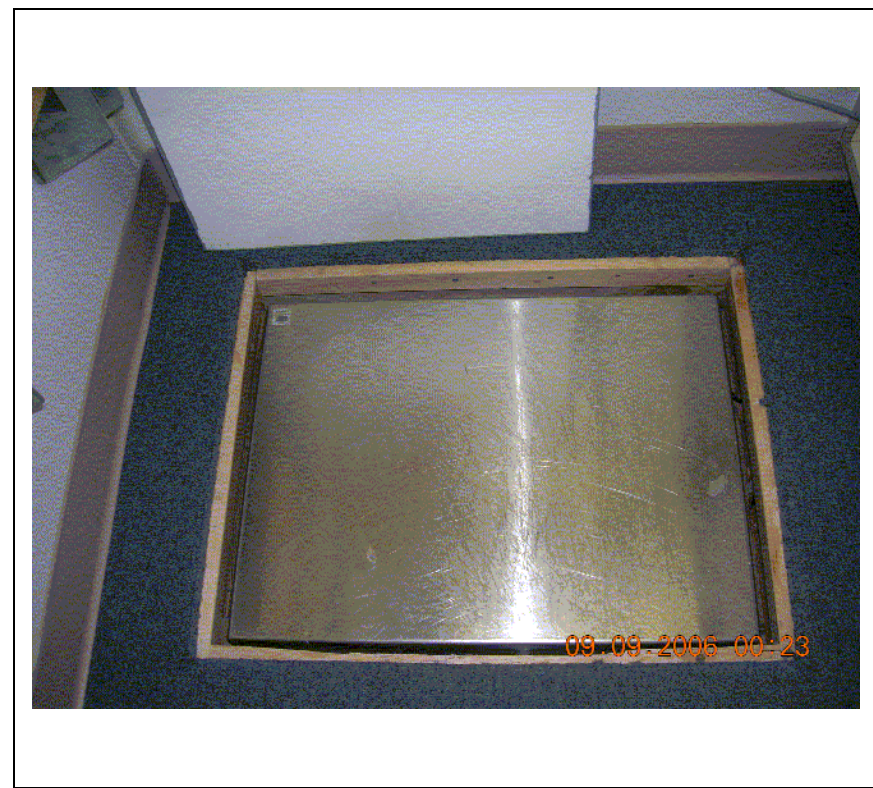

Exhibit 2-2. Digital weight scale-covered

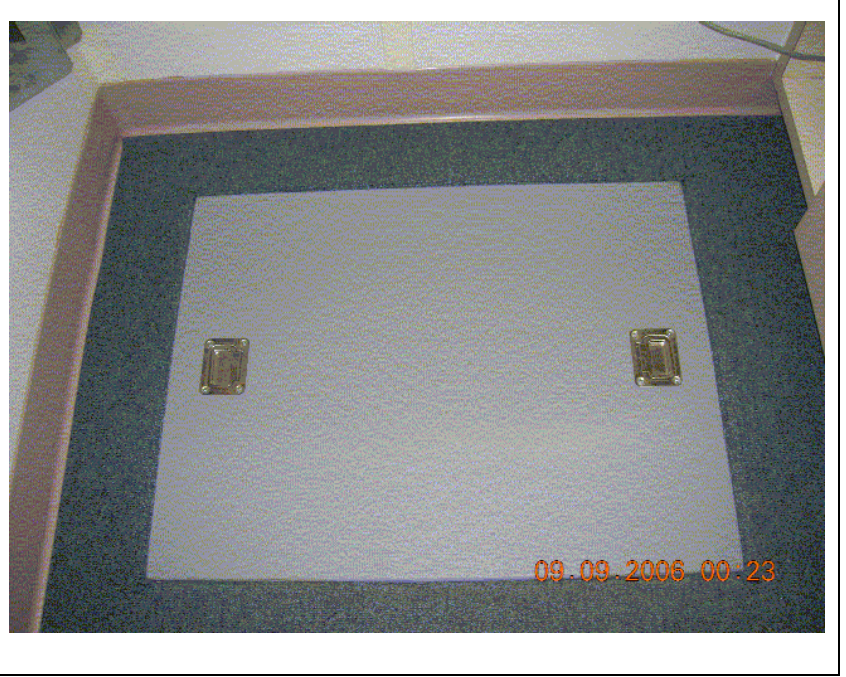

Exhibit 2-4. Digital scale measurement device

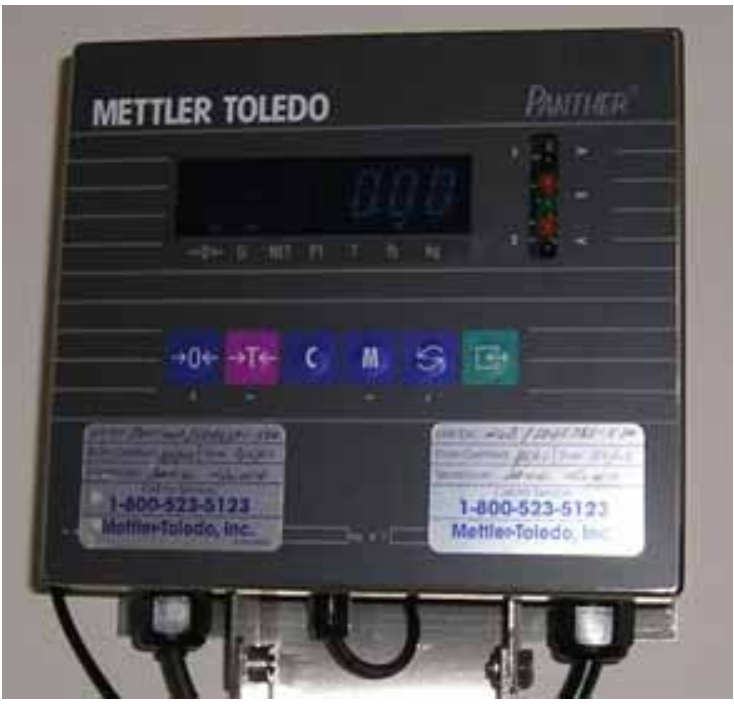




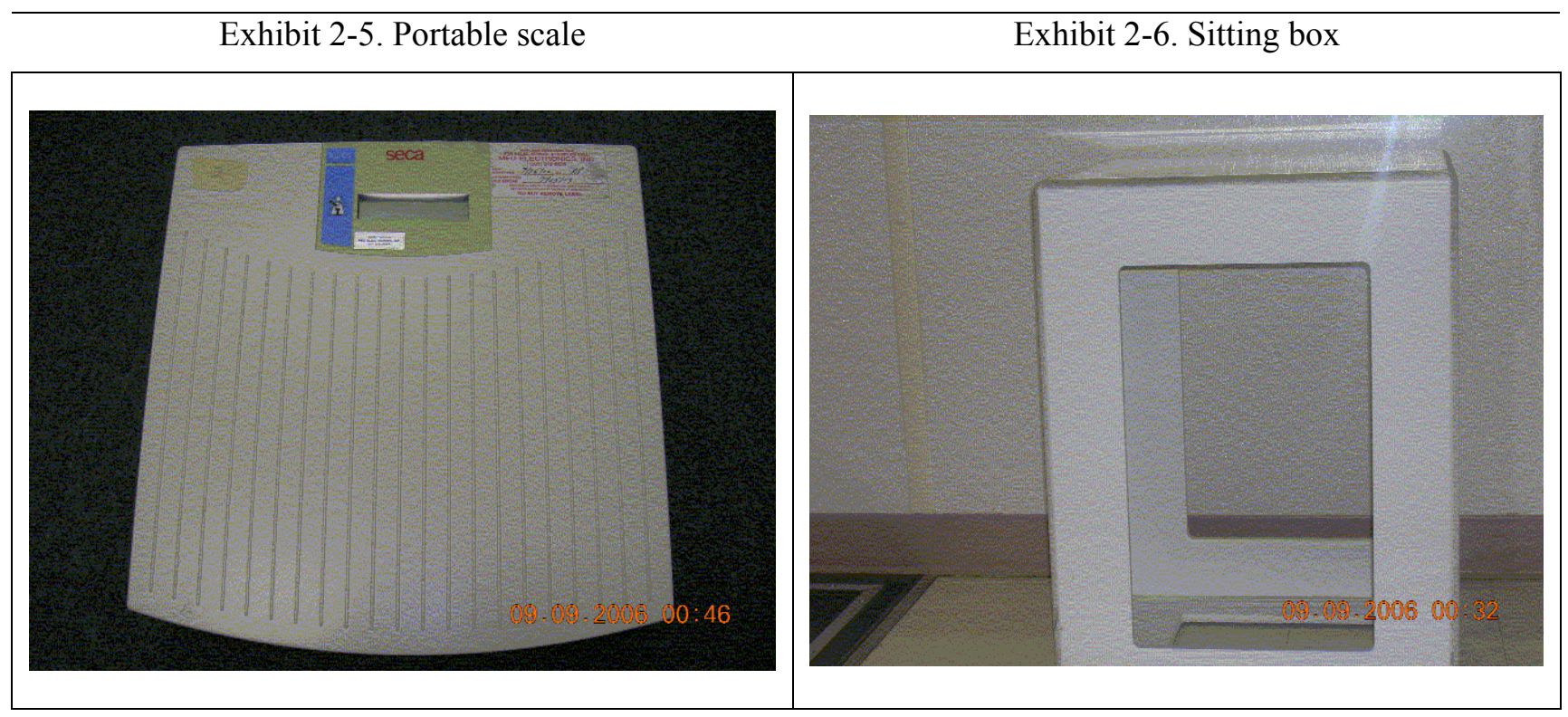

Exhibit 2-7. Stadiometer headpiece and digital measurement device

Exhibit 2-8. Stadiometer calibration rod

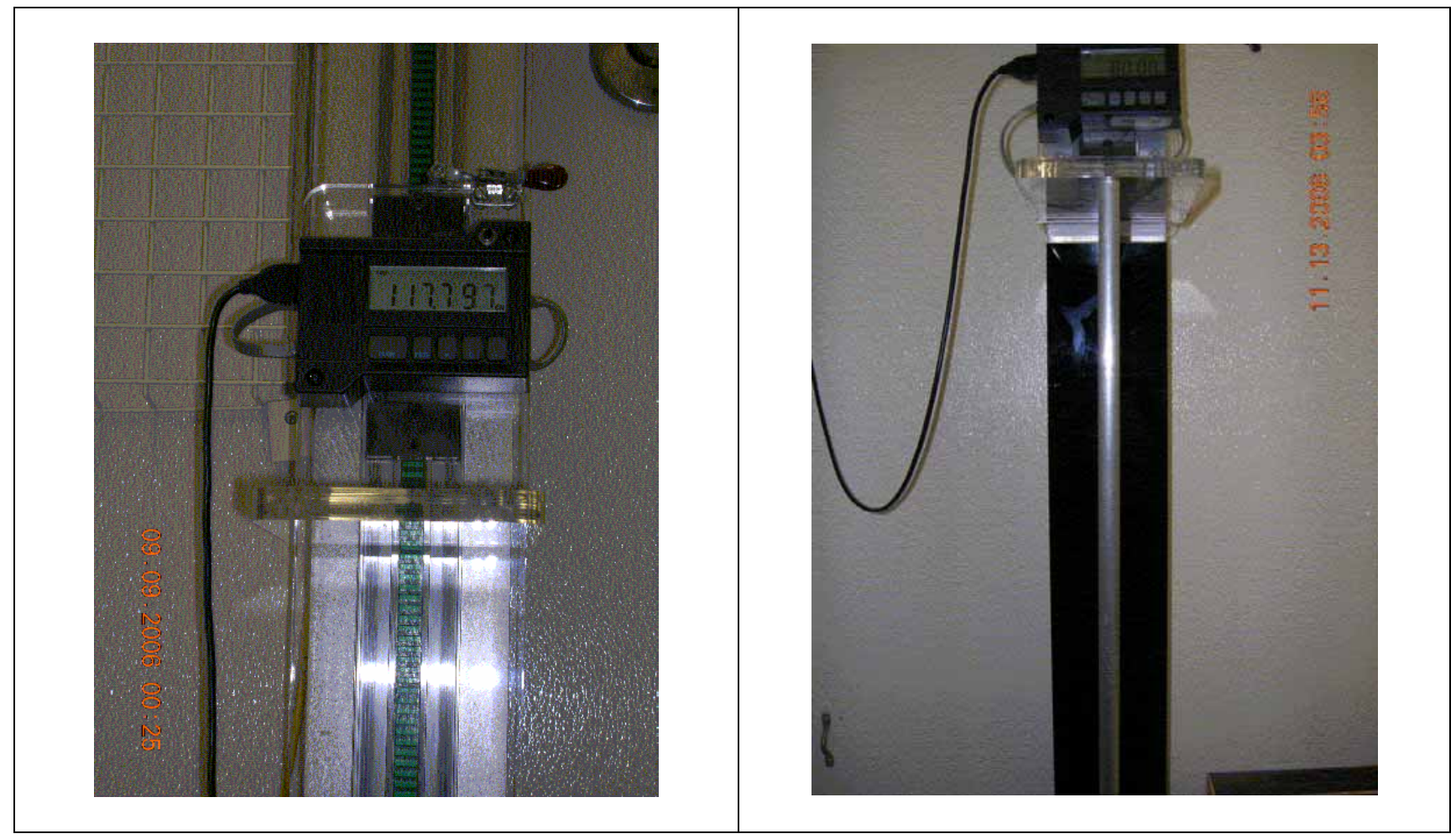



ruler

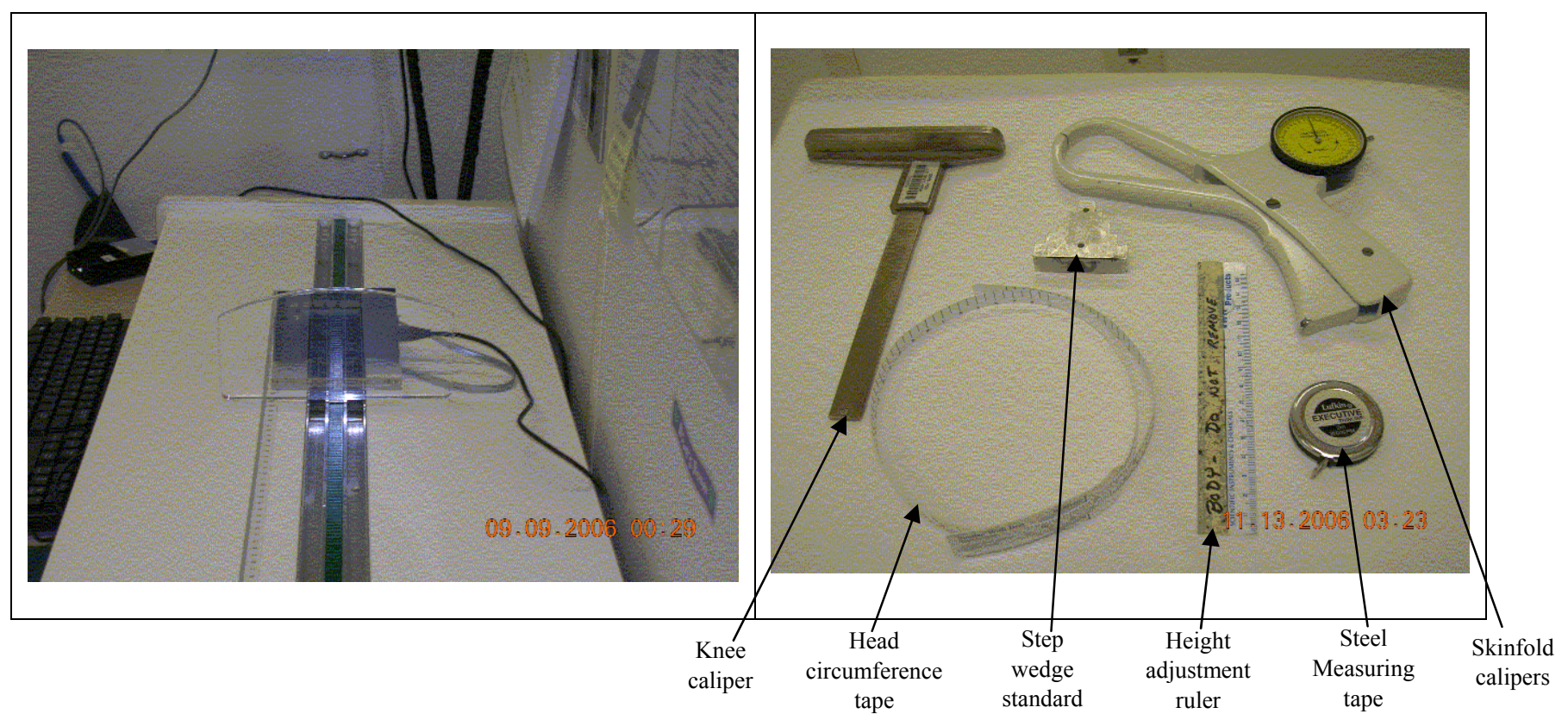

2.2.2 Supplies

- Black/white cosmetic pencil: The technologist and recorder make all body marks on the SP using a wax-based cosmetic pencil. The health tech will use an appropriate color so that the markings are clearly visible on the SP's skin.

- Drop dispenser:

A small dropper bottle is used to dispense baby oil to remove the cosmetic pencil marks.

- Baby oil:

Baby oil is supplied on each MEC for removing cosmetic pencil marks from the SP's skin. A small drop dispenser holds the oil for use during exams. Refills are obtained from a larger bottle of baby oil that is stored in the cabinet drawer.

- Gauze pads:

Each MEC has a supply of $2 \times 2$ inch gauze pads. The pads are used to wipe off cosmetic pencil marks with baby oil.

- Masking tape:

At the end of the anthropometry exam, the technologist tapes the exam gowns to afford privacy to the SP. 
- Absorbent pad (Chux):

- Blunt-edge scissors:

- 9-volt battery:

- AA batteries:

- Alcohol wipes:

- Cotton cloth:

- Bleach disinfectant spray:

- Scrub brush:

- Sewing machine oil:

- Hex key set:

- Screwdrivers:
A clean Chux pad is laid on the infantometer before each use. The pads may also be placed beneath an infant while the guardian removes the child's clothing in preparation for taking measurements.

Stainless steel, blunt-edge scissors are used by the technologist to cut the SP gown pants and shirt in preparation for leg and arm measurements.

The digital measurement devices for the stadiometer and infantometer are powered by 9-volt batteries. The technologist will remove the batteries at the end of a stand and replace them at the beginning of a stand.

Each portable scale is powered by four AA batteries. The technologist will remove the batteries at the end of a stand and replace them at the beginning of a stand.

Prepackaged alcohol wipes will be used daily by the technologist to clean the surface of equipment such as the knee calipers, skinfold calipers, and tape measures.

A cotton cloth is supplied on each MEC for wiping the surface of the aluminum track and digital measurement device of the stadiometer and infantometer.

Bleach disinfectant spray is provided on each MEC for cleaning the surface area of the infantometer, sitting box, and other areas that require disinfection as needed.

A black hand brush is kept on each MEC for cleaning heavily soiled areas as needed.

A 4-ounce bottle of all-purpose, sewing machine oil is supplied on each MEC. The oil is used to lubricate the aluminum track of the stadiometer and infantometer when sliding the head/foot piece becomes difficult.

A 7-piece hex key set is available on each MEC. The hex keys are used to adjust screws on the measurement column and digital measurement device of the stadiometer and infantometer.

A flat and Phillips head screwdrivers are used to open the digital measurement device of the stadiometer and infantometer when changing the batteries. The screwdrivers are stored in the cabinet drawers. 
- Copper clips:

The infantometer and stadiometer contain copper clips in the digital measurement device. The inside of the device may become corroded over time. Therefore, two spare sets of copper clips are kept in a covered plastic cup in the cabinet drawers.

Exhibits 2-11 to 2-13, photographs of anthropometry supplies follow.

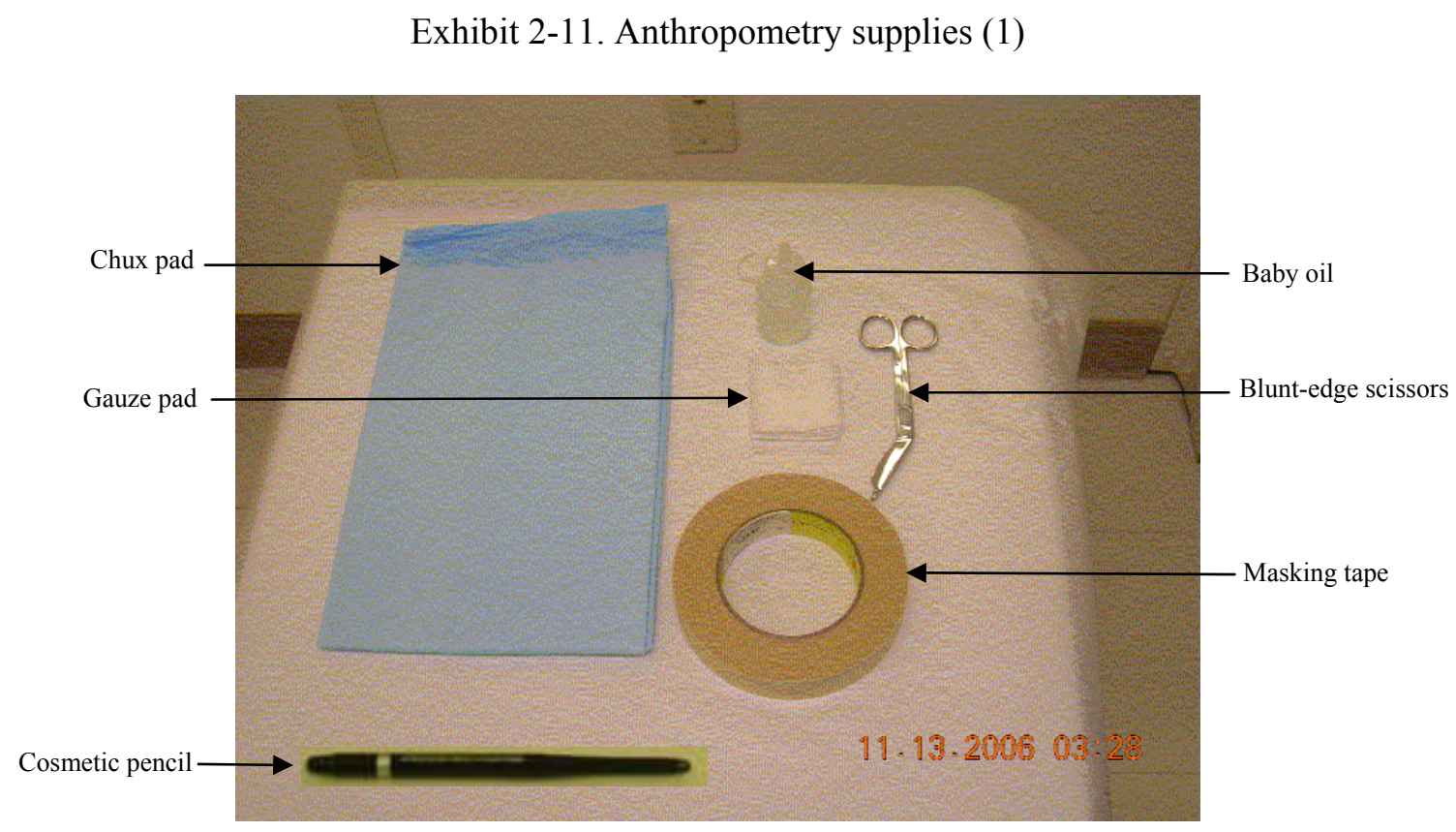

Exhibit 2-12. Anthropometry supplies (2)

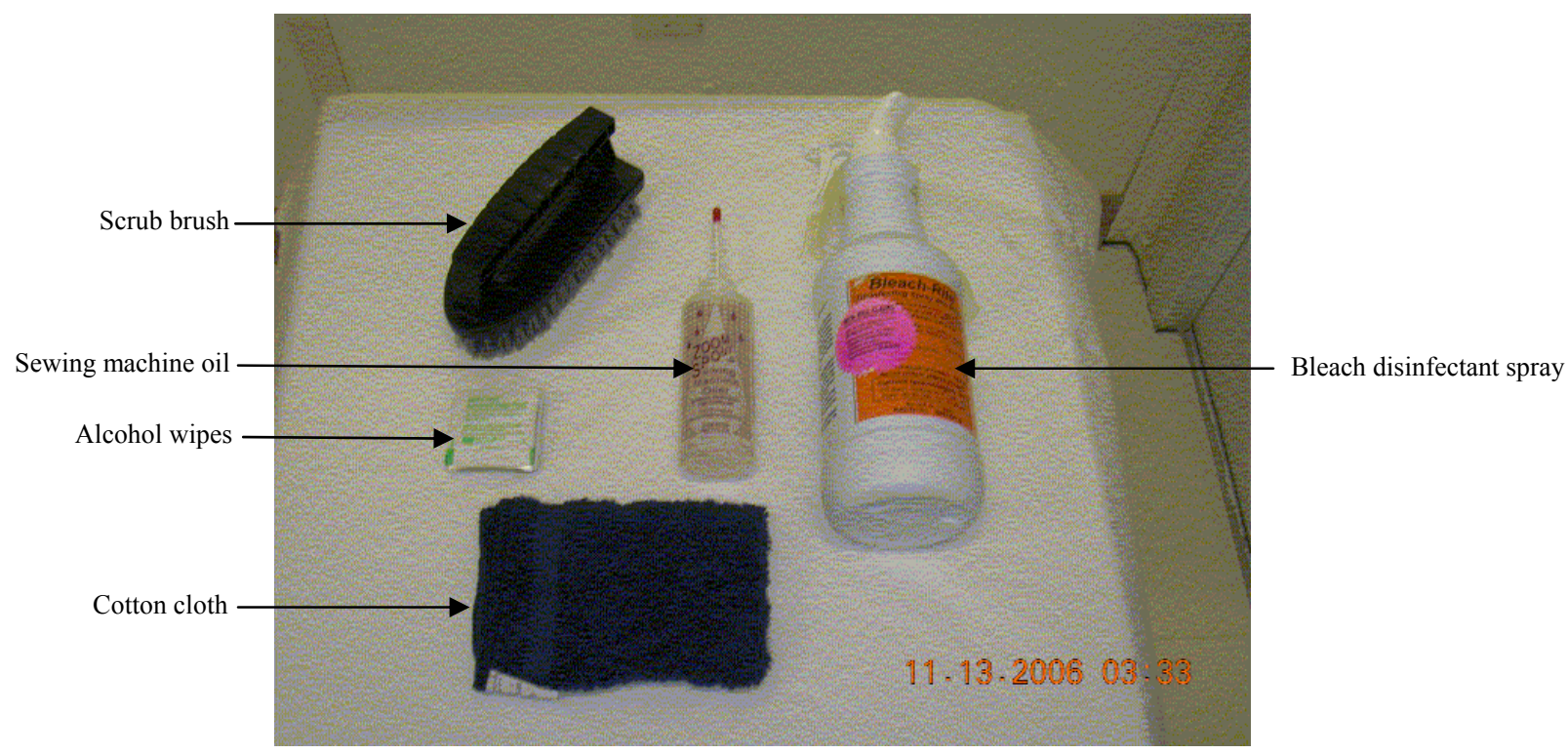


Exhibit 2-13. Anthropometry supplies (3)

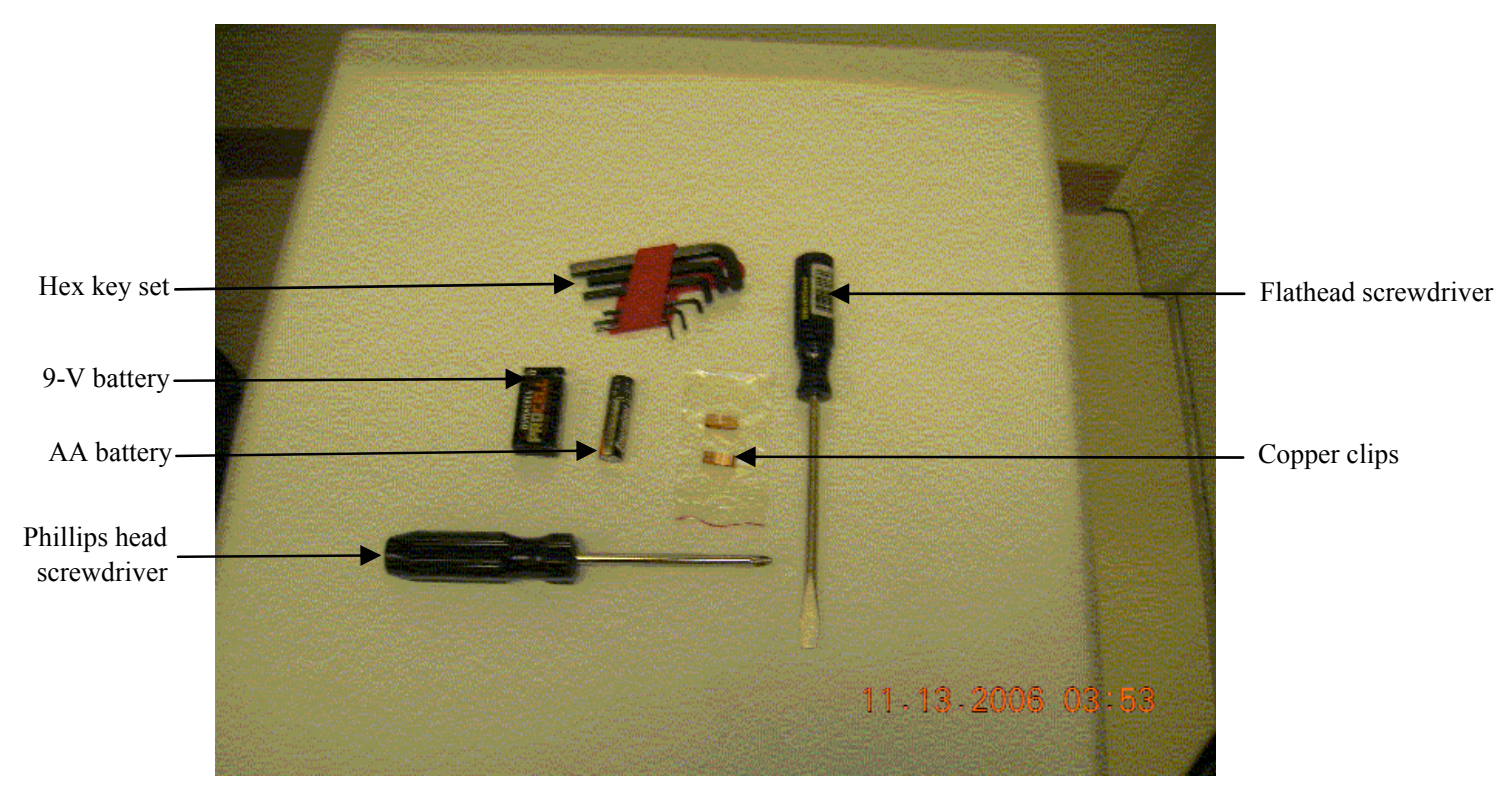

At the beginning and end of each stand, the health technologist will take an inventory of all component-specific equipment and supplies. Any needed items should be noted on the inventory sheet; reported to the chief technologist and MEC manager; and documented in the Unusual Field Occurrence or UFO system (refer to UFO Utility Manual for details).

\subsection{Setup Procedures}

The following procedures describe how to set up the anthropometry equipment and supplies at the start of a stand.

\subsubsection{Calibration Weights}

- Remove any storage material from around the weights.

- Pack the storage material in the anthropometry container that is kept in the MEC belly compartment.

- Use the weights to calibrate the digital weight scale and portable scales (see Chapter 4, Quality Control, Calibration Procedures, Section 4.4 Equipment Calibration). 


\section{Digital Weight Scale}

- Remove the cover from the scale.

- Check that all four feet of the scale base lie on the metal platform; adjust if needed.

- Plug the power cord into the electrical outlet.

- Calibrate the scale using the calibration weights (see Chapter 4, Quality Control, Calibration Procedures, Section 4.4 Equipment Calibration).

- Return the cover to the scale.

\subsubsection{Portable Scale}

- Remove the wooden stick from the cabinet drawer handles and place it in the anthropometry storage container.

- Remove the two portable scales from the cabinet drawers.

- Reconnect or replace the four AA batteries in each scale.

- Calibrate the portable scales using the calibration weights (see Chapter 4, Quality Control, Calibration Procedures, Section 4.4 Equipment Calibration).

- Return the portable scales to the cabinet drawers.

\subsubsection{Sitting Box}

- Remove the strap and foam pad from the box.

- Store the strap and foam pad in the anthropometry storage container.

- Slide the sitting box to the wall with the mirror.

\subsubsection{Stadiometer}

- If not already done, follow the instructions in Section 2.3.4 to separate the sitting box from the stadiometer.

- Replace the 9-volt battery in the digital measurement device for the stadiometer. NOTE: The facility equipment specialist is expected to do this. 
- Calibrate the stadiometer using the calibration rod (see Chapter 4, Quality Control, Calibration Procedures, Section 4.4 Equipment Calibration). When not in use, the rod should be kept in the holding grips on the side of the stadiometer.

- Slide the head piece of the stadiometer to the top of the measurement column.

\subsubsection{Infantometer}

- Remove the strap from the infantometer and store it in the anthropometry storage container.

- Remove the bar code wand and padding from between the foot piece and the end of the infantometer. Store the padding in the cabinet drawers.

- Replace the 9-volt battery in the digital measurement device for the infantometer. NOTE: The facility equipment specialist is expected to do this.

- Calibrate the infantometer (see Chapter 4, Quality Control, Calibration Procedures, Section 4.4 Equipment Calibration).

- Slide the foot piece of the infantometer to the left end of the measurement column.

\subsubsection{Skinfold Caliper}

- If not already done, remove the wooden stick from the cabinet drawers and place it in the anthropometry storage container.

- Remove the two skinfold calipers from the cabinet drawers.

- Calibrate both skinfold calipers using the step wedge standard (see Chapter 4, Quality Control, Calibration Procedures, Section 4.4 Equipment Calibration).

- Place one skinfold caliper in the wall bin beside the computer terminal; return the other caliper to its protective case in the cabinet drawers.

\subsubsection{Knee Caliper}

- If not already done, remove the wooden stick from the cabinet drawers and place it in the anthropometry storage container.

- Remove one knee caliper from the cabinet drawers and place it on a wall hook. 
- If not already done, remove the wooden stick from the cabinet drawers and place it in the anthropometry storage container.

- Remove the insertion tape from the cabinet drawers and place it on a wall hook or in the wall bin beside the computer terminal.

- Remove the steel measuring tape and height adjustment ruler from the cabinet drawers and ensure that they are accessible.

\subsubsection{Supplies}

- If not already done, remove the wooden stick from the cabinet drawers and place it in the anthropometry storage container.

- Ensure that the following supplies are accessible: cosmetic pencils, drop dispenser filled with baby oil, gauze pads, masking tape, and infant blue Chux.

- Hang the blunt-edge scissors on a wall hook.

- Ensure that the following additional supplies are available: alcohol wipes, cotton cloth, bleach disinfectant spray, scrub brush, sewing machine oil, hex key set, screwdrivers, and copper clips.

- Ensure that a small trash can is available in the room.

\subsection{Equipment Care and Maintenance}

Follow the procedures below to ensure that the anthropometry equipment functions properly and remains hygienic throughout the stand.

\section{Start of Stand and Daily}

- Clean the surface of the knee calipers, skinfold calipers, and tape measures with alcohol wipes.

- Clean the white area of the infantometer and sitting box with bleach disinfectant spray. Use paper towels or the scrub brush if necessary.

NOTE: The Formula 409 cleaner that is generally supplied on each MEC can be used to remove dirt, but it is not suitable for use as a disinfectant. 
- Clean the acrylic area of the head/foot piece of the stadiometer and infantometer with bleach disinfectant spray.

- Clean the digital measurement device of the stadiometer and infantometer with a dry cloth or paper towel.

NOTE: Do not allow any fluid to drip into the housing of the digital measurement device.

- Check that the head/foot piece of the stadiometer and infantometer slides smoothly along the measurement column. Clean the aluminum track with a damp cloth or paper towel. As needed, lubricate the track with sewing machine oil - place a few drops of the oil along the track and then slide the head/foot piece up and down the column.

\subsection{Equipment Malfunctions}

All equipment malfunctions or repair needs must be reported promptly to the chief technologist, MEC manager, and home office component specialist. If the issue is computer-related and cannot be resolved by the chief tech and/or MEC manager, please contact the home office ISIS support staff.

Equipment issues should be documented in the Unusual Field Occurrence (UFO) system and Equipment Tracking System (ETS) as appropriate (refer to the UFO Utility Manual and ETS User Guide for details). The component specialist will contact the equipment manufacturer for assistance.

A complete set of backup anthropometry equipment is kept at the home office for training and ISIS testing purposes. Some backup equipment for the anthropometry component is kept in each MEC for use until the malfunctioning equipment can be repaired or replaced. Specifically, two portable scales will serve as substitutes for the digital weight scale; spare skinfold and knee calipers are kept in the cabinet.

The ISIS anthropometry software application can be used in a manual data entry mode. This feature is especially valuable in the event that the stadiometer, infantometer, or digital weight scale fails to transfer data directly to ISIS. If the ISIS system fails to capture one or more data points, the examiner should complete a UFO, including the SP ID, the missing values, and a description of the problem encountered during the exam. 
If ISIS is completely unavailable for use during a session, the examiner can still perform the component. The recorder should record all measurements on the Body Measures Recording Form (see Appendix A). Several copies of the form should be kept in the cabinet drawer. MEC staff can obtain additional copies in the "Blank Forms Directory" on the MEC staff lounge computer or via an email request to the home office component specialist. Recorders should print all hard-copy form data legibly. The Body Measures Recording Form can be faxed or mailed to the home office. The component specialist will then complete a Back-End-Edit-Request (BEER) to add the data to the study database.

\subsection{Packup Procedures}

At the end of the stand, follow the procedures described below to pack up the anthropometry equipment for travel to the next stand. The anthropometry storage container will need to be retrieved from the MEC belly compartment and brought to the exam room for packup.

\subsubsection{Calibration Weights}

- Use the weights to calibrate the digital weight scale and portable scales (see Chapter 4, Quality Control, Calibration Procedures, Section 4.4, Equipment Calibration).

- Place the storage material from the anthropometry container around the weights.

\subsubsection{Digital Weight Scale}

- Calibrate the scale using the calibration weights (see Chapter 5, Quality Control, Calibration Procedures, Section 5.4, Equipment Calibration).

- Unplug the power cord from the electrical outlet.

- Place the cover on the scale.

- Lower the seat located above the scale. 
- Disconnect the four AA batteries in each portable scale.

- Leave the scales in the cabinet drawers.

\subsubsection{Sitting Box}

- Remove a strap and square piece of foam padding from the anthropometry storage container.

- Slide the sitting box against the stadiometer and insert the foam padding as a buffer between the sitting box and the stadiometer.

- Place the strap around two legs of the box and attach the ends of the strap to the designated wall hooks.

\subsubsection{Stadiometer}

- If not already done, follow the instructions in Section 2.6.4 to secure the sitting box to the stadiometer.

- Remove the 9-volt battery from the digital measurement device of the stadiometer. NOTE: The facility equipment specialist is expected to do this.

- Lower the stadiometer head piece to the sitting box.

- Check that the screws at the top of the measurement column are secured. If necessary, tighten the screws using the hex key set.

\subsubsection{Infantometer}

- Remove a strap and padding from the anthropometry storage container.

- Remove the 9-volt battery from the digital measurement device of the infantometer. NOTE: The facility equipment specialist is expected to do this.

- Slide the foot piece to the left end of the measurement column.

- Place the strap around the metal base of the foot piece and attach the ends of the strap to the designated wall hooks. 
- Wrap the bar code wand in the padding and place this between the foot piece and the end of the infantometer.

\subsubsection{Skinfold Caliper}

- Place the skinfold calipers in their protective case and store in the cabinet drawers.

\subsubsection{Knee Caliper}

- Place the knee caliper in the cabinet drawers.

\subsubsection{Measurement Tapes and Ruler}

- Place the plastic insertion tape, steel measuring tape, and height adjustment ruler in the cabinet drawers.

\subsubsection{Supplies}

- Place the following supplies in the cabinet drawers: cosmetic pencils, drop dispenser filled with baby oil, gauze pads, masking tape, infant blue Chux, blunt-edge scissors, and alcohol wipes.

- To avoid spillage during travel, ensure that the large, refill bottle of baby oil is upright and in a plastic bag in the cabinet drawer.

- Ensure that the following additional supplies remain stored in the cabinet drawers: cotton cloth, bleach disinfectant spray, scrub brush, sewing machine oil, hex key set, screwdrivers, and copper clips.

- Place the computer key board and mouse (with cables still attached) in the top drawer of the cabinet.

- Remove the wooden stick from the anthropometry storage container and insert it in the cabinet drawer handles.

- Discard any trash in the room. 


\section{EXAMINATION PROTOCOL}

\section{1 $\quad$ Eligibility Criteria}

All NHANES sample persons (SPs) are eligible for the Anthropometry or Body Measurement component in the mobile examination center (MEC). The anthropometry protocol requires specific measurements depending on the age of the SP. Table 3-1 lists the required measurements by age category.

Table 3-1. Body measurements by age category

\begin{tabular}{|c|c|c|c|c|}
\hline Birth+ & $2 \mathrm{mo}+$ & $2 \mathrm{yr}+$ & $4 \mathrm{yr}+$ & $8 \mathrm{yr}+$ \\
\hline \multirow{11}{*}{$\begin{array}{l}\text { Weight } \\
\text { Recumbent length } \\
\text { Head } \\
\text { circumference }\end{array}$} & Weight & Weight & Weight & Weight \\
\hline & Recumbent length & $\begin{array}{l}\text { Recumbent length } \\
\text { (through } 47 \mathrm{mos} \text { ) }\end{array}$ & & \\
\hline & $\begin{array}{l}\text { Head circumference } \\
\text { (through } 6 \text { months) }\end{array}$ & & & \\
\hline & & Standing height & Standing height & Standing height \\
\hline & & & & Upper leg length \\
\hline & Upper arm length & Upper arm length & Upper arm length & Upper arm length \\
\hline & Arm & Arm & Arm & Arm \\
\hline & & Waist & Waist & Waist \\
\hline & & circumference & circumference & circumference \\
\hline & Triceps skinfold & Triceps skinfold & Triceps skinfold & Triceps skinfold \\
\hline & $\begin{array}{l}\text { Subscapular } \\
\text { skinfold }\end{array}$ & $\begin{array}{l}\text { Subscapular } \\
\text { skinfold }\end{array}$ & $\begin{array}{l}\text { Subscapular } \\
\text { skinfold }\end{array}$ & $\begin{array}{l}\text { Subscapular } \\
\text { skinfold }\end{array}$ \\
\hline
\end{tabular}

\subsection{Pre-examination Procedures}

If possible, prepare the room for the examination before the SP enters the room. The recorder will confirm that all supplies needed for the exam are available and accessible: cosmetic pencil, gauze pads, baby oil, masking tape strips, scissors, steel measuring tape, skinfold calipers, head circumference tape, and absorbent pads.

When the SP enters the room, introduce yourself and the recorder and close the exam room curtain. The recorder will open the anthropometry component in ISIS, log the SP into the ISIS system, 
and direct the SP to the scale. The anthropometry component requires no formal or standard script. However, the examiner will provide a brief introduction to the examination, such as:

"In this room we will take a number of body measurements such as weight and height. We will also measure the length of your arm and leg, and take some circumference and skinfold measurements."

Prior to initiating the first measurement, ask the SP to remove any items from his or her pockets, and set these and other belongings on the infantometer.

\subsection{General Guidelines for Measuring and Recording}

Follow the guidelines below when taking anthropometric measurements and subsequently recording the data into ISIS:

1. Always tell the SP what you are going to do before you do it. Explain what you are doing and why, such as when locating the leg tendon in the groin area to measure the upper leg length; or before adjusting the pants down to measure the waist circumference. Remain unaffected by tattoos, piercings, etc. and do not comment about the SP's body. Maintain professionalism at all times.

2. Measure the right side of the body. If the SP has a physical disability or abnormality on the right side, the examiner should still attempt to measure the right side. Only take measurements on the left side when the SP has a cast, prosthesis, or amputation on the appropriate right limb; or for some other reason the measurement cannot be taken on the right side.

3. Turn the SP in the direction needed for a given measure. Do not move yourself around the SP instead. This promotes efficiency during the examination by saving time and avoiding unnecessary movement on the part of the examiner.

4. Position the zero end of the measuring tape below the measurement value. With respect to circumference measures, do not take any measurement readings with the zero end of the tape placed above the section of the tape with the result.

5. Avoid parallax when taking measurement readings. Parallax describes the phenomenon where an observer reads a different value on a measuring device depending on the angle from which it is viewed. Parallax is a common cause of data error especially for measurements obtained using the skinfold calipers and measurement tape. The examiner should read the measurement with his or her line of sight directly in front of the value rather than at an angle or from even slightly off to the side. 
6. Record all measurements (except skinfolds) to the nearest tenth of a centimeter $(0.1 \mathrm{~cm})$. Record skinfold measurements to the nearest tenth of a millimeter $(0.1$ mm). For quality control purposes, the computer will read each number aloud immediately following data entry. ISIS will also alert the recorder to any values that fall below the 1st percentile or above the 99th percentile based on the 1998-1994 (NHANES III) data collection period. Always verify the result before advancing to the next measure.

\subsection{Examination Procedures}

This section describes the anthropometry examination procedures. As previously shown in Table 3-1, the protocol includes a total of 10 body measures. Exam staff will collect a minimum of three, and a maximum of eight measures, depending on the age of the participant.

\subsubsection{Weight}

Participants will be weighed in kilograms using a digital weight scale. At the end of the examination ISIS will display the weight in both kilograms and pounds for the recorder to share with the SP if he or she wants to know the result. Participants should wear the standard MEC examination gown, which consists of a disposable shirt, pants, and slippers. SPs should wear only underpants beneath the gown. Infants should wear only diapers. The procedures for obtaining the weight measurement are as follows:

1. Position the SP: After the examiner briefly introduces the exam to the SP, the recorder will direct the SP to stand in the center of the scale platform facing the recorder, hands at sides, and looking straight ahead.

2. Capture the result: After the SP is correctly positioned and the readout on the digital measurement device becomes stable, the recorder will click the Get Weight button on the screen to capture the result into ISIS.

In addition, exam staff should follow the procedures described below when applicable:

- Small children: Infants and toddlers who cannot stand alone on the scale will be weighed with the assistance of an adult. Either the guardian or the examiner will stand alone on the scale while the recorder clicks the Tare button on the ISIS screen. This sets the scale readout to zero. The child is then handed to the adult on the scale. In this way the scale will read only the child's weight. The 
recorder will click Get Weight to transfer this result to ISIS, and Reset to undo the tare setting. See also Sections 3.5 and 4.2.2.

- Casts or prostheses: If you successfully obtained the weight but the SP had a cast or medical prostheses, add the Medical Appliance (MA) code in ISIS. See also Sections 3.5 and 4.2.1.

- Street clothes: If you successfully obtained the weight but the SP wore street clothes instead of the MEC examination gown, add the Clothing (CL) code in ISIS. Do not use this code for infants wearing diapers or a t-shirt.

Weight data collected with the SP wearing shoes on the scale will be considered invalid. Therefore, if the SP wears shoes to the exam ask him or her to remove them before stepping on the scale. If the SP refuses to remove the shoes in order to step on the scale, code the weight measure as Could Not Obtain (CNO). See also Sections 3.5 and 4.2.1.

- Portable scales: Each MEC has two portable scales that are kept in the cabinet drawers. Turn on the scale on by pressing the "On" button, and position the SP on the portable scale in the same manner as for the digital weight scale. Call the weight to the recorder, who will manually enter the number into the Weight field on the ISIS screen. Use the portable scales in three situations:

1. If weight exceeds $440 \mathrm{lbs}$;

The digital weight scale can accurately weigh up to a maximum of 440 pounds. If the examinee weighs more than this, obtain the weight using two portable scales:

- $\quad$ Have the SP stand with one foot on each portable scale.

- $\quad$ Combine the two results to approximate the weight.

- $\quad$ Manually enter the total in the Weight field on the screen.

- If the SP exceeds the capacity of both portable scales (440lb each), code the weight measure as Exceeds Capacity (EC) in ISIS (see Section 4.2.1).

2. If the digital weight scale malfunctions; or

3. In the event of a power outage. 


\subsubsection{Recumbent Length}

The anthropometry protocol requires a stature measurement in the form of recumbent length for all children less than 4 years of age (birth to 47 months). Recumbent length is measured using an infantometer with a fixed head piece and horizontal backboard, and an adjustable foot piece.

\section{Position the SP}

Lay the child on the infantometer on top of a Chux pad with the feet toward the foot piece and the head against the fixed head piece. If the child is not wearing a MEC examination gown, ask the parent or guardian to remove the child's clothes except for the diaper or underpants. The parent can set the child on a Chux pad on top of the sitting box as a changing area.

Placing infants and small children in a recumbent position often generates a sense of insecurity and consequently invokes a crying response. To help encourage and comfort the child, the parent or guardian should stand between the examiner and recorder, make eye contact, and talk to the child throughout the procedure.

The recorder supports the child's head while the examiner positions the feet and ensures that the head lies in the Frankfort horizontal plane described in Section 3.4.4. Apply gentle traction to bring the top of the head in contact with the fixed headpiece. Secure the child's head in the proper alignment by lightly cupping the palms of your hands over the ears. Align the child's legs by placing one hand gently but with mild pressure over the knees. With the other hand, slide the foot piece to rest firmly at the child's heels. The toes must point directly upward with both soles of the feet flexed perpendicular against the acrylic foot piece. To encourage the child to flex the feet, run the tip of your finger down the inside of the foot.

NOTE: If you are unable to achieve both legs outstretched in the correct position, make certain at least one leg is straight with the foot flexed against the foot piece. However, only position with one leg as an exception for extremely fussy children. 


\section{Capture the Result}

When the child is correctly positioned, click the "Get" button on the screen to capture the result in ISIS. Release the child's feet as you hold the foot piece in position. Wait for the computer to repeat the measurement aloud. After verifying the correct value, have the parent or guardian remove the child from the infantometer. Slide the foot piece to the end of the measurement column in preparation for the next participant.

In the event of a power outage or if the infantometer malfunctions, position the child as described above and obtain the recumbent length using the tape measure mounted on the right side of the measurement column. Call the result to the recorder, who will manually enter this number in the appropriate ISIS field.

\subsubsection{Head Circumference}

The circumference of the head is measured on children from birth through 6 months of age. Follow the steps below to obtain the head circumference measurement:

1. Position the SP: Instruct the parent (or guardian) to stand holding the child over the parent's left shoulder or else sit on the white box with the child in the parent's lap. Ask the parent to remove any of the child's hair ornaments or braids.

2. Take the measurement: Place the head circumference tape around the child's head so that the tape lies: across the frontal bones of the skull; slightly above the eyebrows; perpendicular to the long axis of the face; above the ears; and over the occipital prominence at the back of the head (Exhibit 3-1). Move the tape up and down over the back of the head to locate the maximal circumference. Tighten the insertion tape so that it fits snugly around the head and compresses the hair and underlying soft tissues. Measure the circumference to the nearest $0.1 \mathrm{~cm}$. Since the head circumference tape shows both centimeters and inches, check that you take the measurement using the metric scale.

3. Record the result: Call the result to the recorder, who will enter this number on the ISIS screen. Remove the head circumference tape. Proceed to the next measure for SPs in this age group (birth through 6 months), the upper arm length. 
Exhibit 3-1. Head circumference tape position

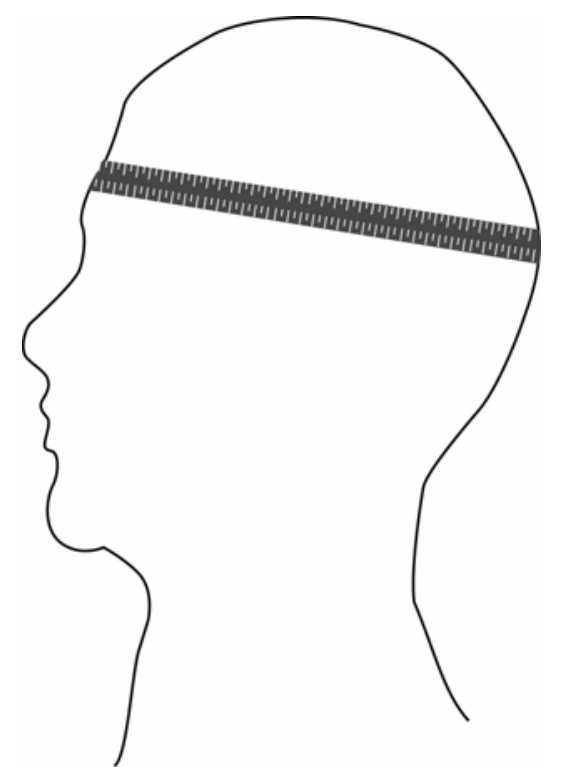

\subsubsection{Standing Height}

Standing height is an assessment of maximum vertical size. This stature measurement is collected on all SPs aged 2 years and older who are able to stand unassisted. Standing height is measured using a stadiometer with a fixed vertical backboard and an adjustable head piece.

\section{Position the SP}

Direct the SP to the stadiometer platform. Ask him or her to remove any hair ornaments, jewelry, buns, or braids from the top of the head. Exhibit 3-2 on page 3-9 depicts the correct position for the measurement of standing height. First, have the SP stand up straight against the backboard with the body weight evenly distributed and both feet flat on the platform. Instruct the SP to stand with the heels together and toes apart. The toes should point slightly outward at approximately a $60^{\circ}$ angle. Check that the back of the head, shoulder blades, buttocks, and heels make contact with the backboard.

NOTE: Depending on the overall body conformation of the individual, all four contact points - head, shoulders, buttocks, and heels - may not touch the stadiometer backboard. For example, frequently elderly SPs may have kyphosis, a forward curvature of the spine that appears as a hump at the upper back. In particular, dowager's hump is a form of kyphosis that creates a hump at the back of the 
neck. Additionally, some overweight SPs cannot stand straight while touching all four contact points to the backboard. In such instances it is important to obtain the best measurement possible according to the protocol. If you cannot ensure that the SP's trunk remains vertical above the waist, the arms and shoulders are relaxed, and the head lies in the Frankfort plane, then call "NS" to the recorder who will capture the measurement and add the Not Straight comment beside the appropriate stature measure in ISIS (Section 4.3.2).

Second, align the head in the Frankfort horizontal plane. The head is in the Frankfort plane when the horizontal line from the ear canal to the lower border of the orbit of the eye is parallel to the floor and perpendicular to the vertical backboard (Exhibit 3-2). Many people will assume this position naturally, but for some SPs the examiner may need to gently tilt the head up or down to achieve the proper alignment. Instruct the SP to look straight ahead.

Next, lower the stadiometer head piece so that it rests firmly on top of the participant's head, with sufficient pressure to compress the hair. Instruct the SP to stand as tall as possible, take a deep breath, and hold this position. The act of taking a deep breath helps straighten the spine to yield a more consistent and reproducible stature measurement. Notice that the inhalation will cause the headpiece to rise slightly.

Some SPs have hair styles such as a barrette, bun, or braid that will interfere with the placement of the stadiometer head piece. Other SPs may refuse to remove their shoes for the height measurement. In these cases, while the SP remains positioned on the stadiometer platform, the examiner will measure the hair piece and/or the shoe heel with the $15 \mathrm{~cm}$ height adjustment ruler. The recorder will enter this number in the Height Correction Above/Below Waist field on the screen (Section 4.3.1). ISIS will use this correction factor to automatically calculate an adjusted height value.

\section{Capture the Result}

While the SP is correctly positioned and holding the breath, call "Get" to the recorder, who will click the Get button on the ISIS screen to capture the result. Tell the SP to release the breath as you hold the head piece in position. Wait for the computer to repeat the measurement aloud. After verifying the correct value, have the SP relax and step away from the stadiometer. Slide the head piece to the top of the measurement column and secure it in place with the brake lever, in preparation for the next participant. 
In the event of a power outage or if the stadiometer malfunctions, slide the headpiece to the top of the measurement column and obtain the height using the tape measure mounted on the right side of the measurement column. Call the result to the recorder, who will manually enter this number in the appropriate ISIS field.

Exhibit 3-2. Standing height position

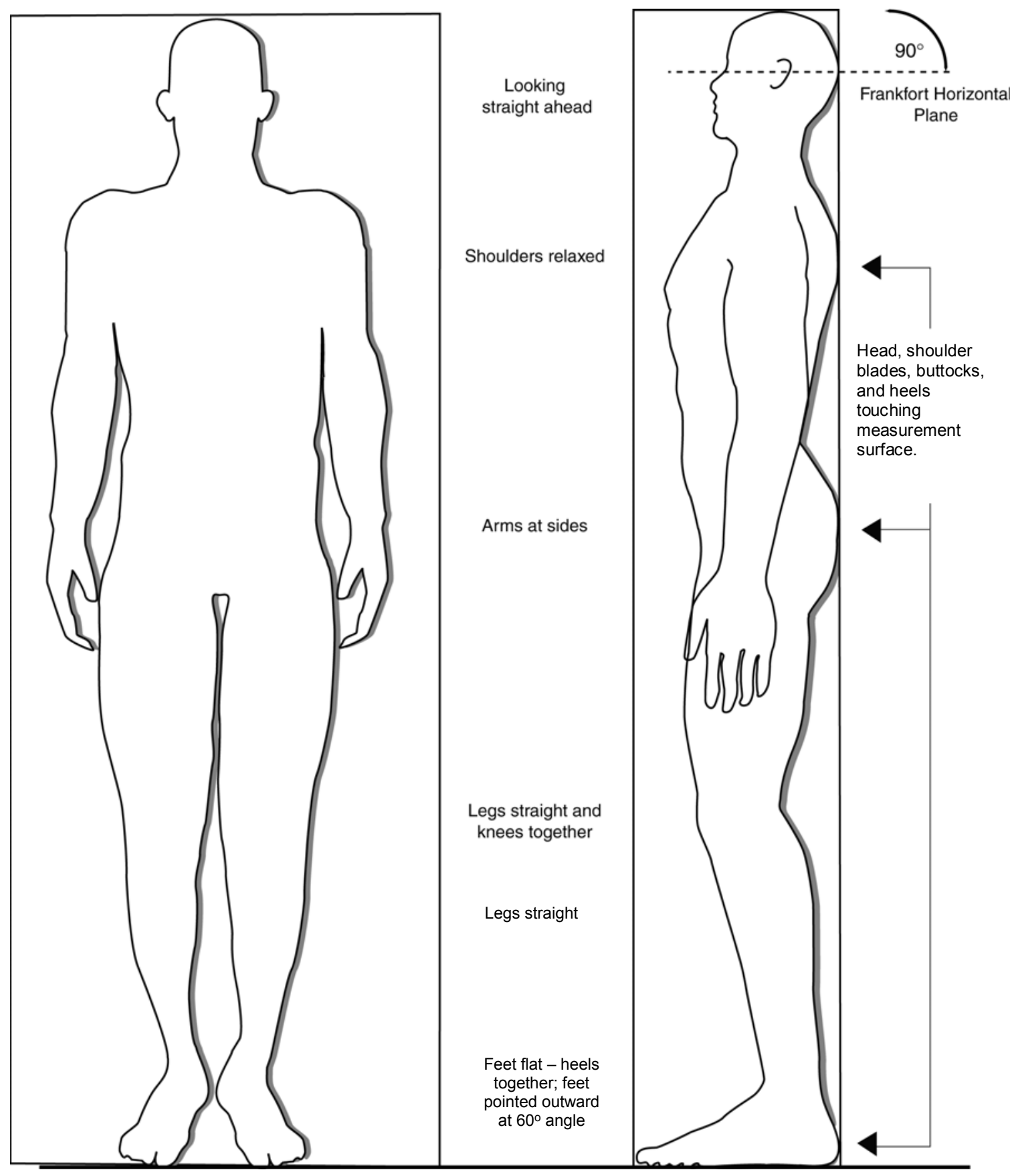




\subsubsection{Upper Leg Length}

Following the weight and height measures, the length of the upper leg will be collected on participants aged 8 years and older. This section describes the procedures to obtain the upper leg length measurement:

1. Position the SP: Ask the SP to sit on the white box with the right knee bent at a $90^{\circ}$ angle. Tell the SP that you are going to cut the right sleeve and pant leg of the examination gown. Assure him or her that you will tape these areas closed at the end of the exam. Using the blunt-edge scissors cut the right sleeve to the edge of the shoulder and the pant leg to the edge of the pocket.

2. Mark the measurement site: Hand the scissors to the recorder in exchange for the knee caliper. Position the knee caliper as if you were measuring the breadth of the patella. Place the caliper blades against the distal end of the femur on either side of the patella. The horizontal bar of the caliper should touch or lie close to the anterior surface of the thigh, proximal to the patella. With a cosmetic pencil mark a line along the proximal border of the patella on the anterior surface of the thigh. Use the edge of the horizontal bar of the caliper as a guide.

3. Take the measurement: Instruct the participant to sit up straight and tell him or her what you will do next. For example, say that you are going to locate the muscle tendon where the leg meets the rest of the body. Place the zero end of the steel measuring tape at the inguinal crease, just below the anterior superior iliac spine (Exhibit 3-3). Depending on the SP, the examiner may need to lift folds of fat tissue in order to locate this crease. Check that the zero end of the tape is properly positioned: place your thumb firmly over the zero end of the tape and ask the SP to lift the thigh slightly off the box. If the tape is in the correct position at the inguinal crease, you will feel a definite tightening of the tendon.

Pull the pant leg slightly to smooth out any gathers. Ask the SP to relax the right leg and remind him or her to sit up straight. Starting from the inguinal crease, extend the tape down the anterior midline of the thigh to the cosmetic pencil mark. Take the measurement to the nearest $0.1 \mathrm{~cm}$.

4. Record the result: Call the result to the recorder, who will enter this number on the ISIS screen. Instruct the SP to come off the box, stand on the floor, and turn to face away from you. To improve the overall flow of the examination, mark the site needed for the subscapular skinfold (Section 3.4.10.2) immediately after completing the upper leg length measurement. 
Exhibit 3-3. Upper leg length measurement position

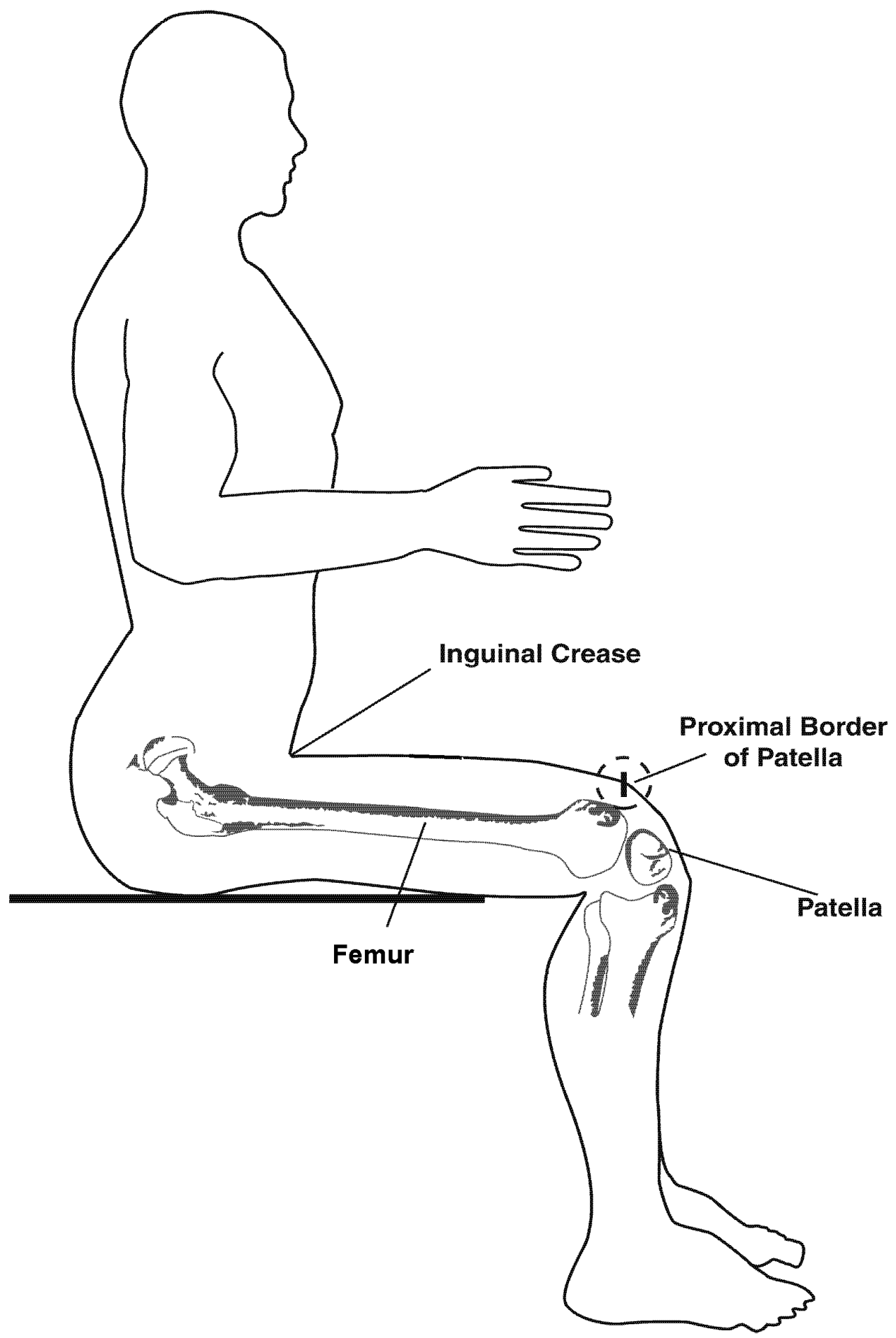




\subsection{6}

\section{Upper Arm Length}

In this study, the length of the upper arm is measured in part to obtain reliable arm circumference and skinfold measurements (Sections 3.4.8 and 3.4.10, respectively). Exam staff will measure the upper arm length on participants aged 2 months and older. To expedite the examination, mark the site for the subscapular skinfold measurement (Section 3.4.10.2) just before initiating the procedures described below:

1. Position the SP: Direct the SP to turn away from you. Ask him or her to stand upright with the weight evenly distributed on both feet, the right arm bent $90^{\circ}$ at the elbow, and the right palm facing up. Demonstrate the correct position if necessary.

2. Mark the measurement site: Locate the end of the spine of the right scapula by following the scapula out to the arm until it makes a sharp V-turn to the front of the body. Using the cosmetic pencil, make a horizontal line on the uppermost edge of the posterior border of the spine extending from the acromion process (see Exhibit $3-4)$.

3. Take the measurement: Hold the zero end of the measuring tape at this mark and extend the tape down the posterior surface of the arm to the tip of the olecranon process, the bony part of the mid-elbow (Exhibit 3-8). Take the measurement to the nearest $0.1 \mathrm{~cm}$. IMPORTANT: The tape must be centered on the posterior surface of the arm. Exhibit 3-5 shows the correct placement of the measuring tape centered on the posterior surface of the arm; whereas Exhibit 3-6 shows the measuring tape placed incorrectly.

4. Record the result: Call the result to the recorder, who will enter this number on the ISIS screen. Keep the measuring tape in position.

5. Mark the midpoint: After the recorder enters the result, the application will divide the value in half to calculate the midpoint of the measured length. A computergenerated voice will repeat the measurement aloud and call out the midpoint. While the examiner holds the tape in place, the recorder will make a horizontal mark at the midpoint and cross this mark with a perpendicular line (Exhibit 3-7). The examiner may need to remove the tape to allow the recorder to complete the cross mark $(+)$. IMPORTANT: The vertical line must be centered on the posterior surface of the arm. This mark defines the site at which both the arm circumference and the triceps skinfold will be measured. Finally, tell the SP to relax the right arm. Proceed to the arm circumference measure. 
Exhibit 3-4.

Marking spine extending from acromion process

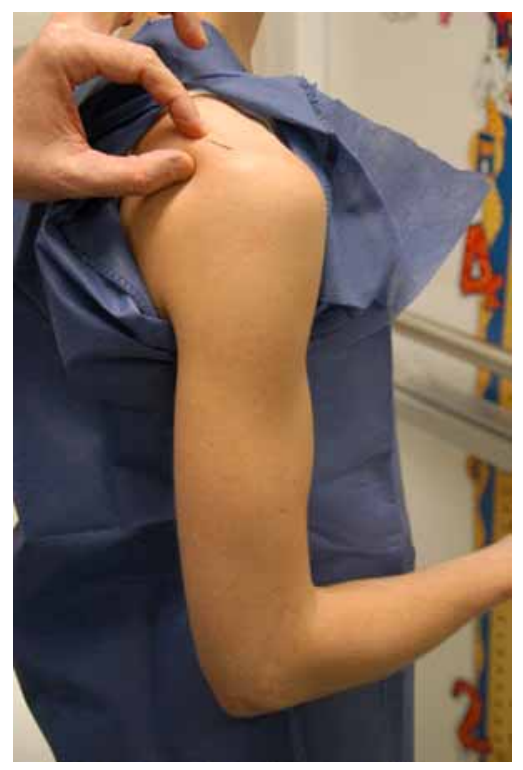

Exhibit 3-6.

Incorrect tape placement for upper arm length

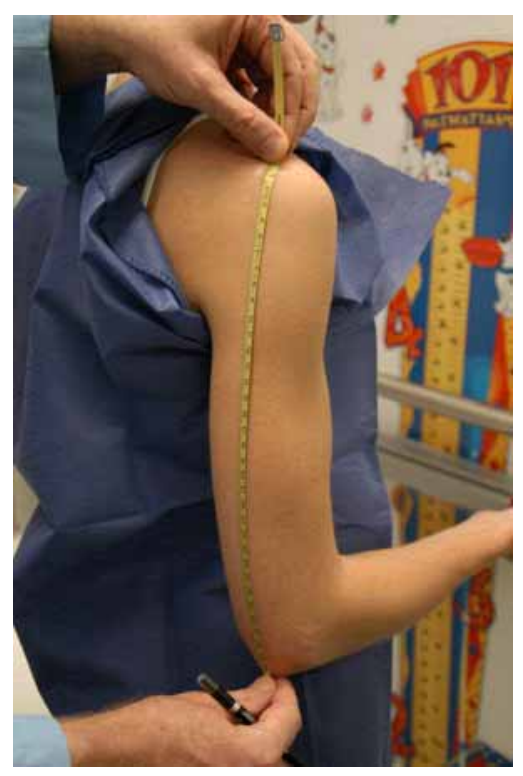

Exhibit 3-5.

Correct tape placement for upper arm length

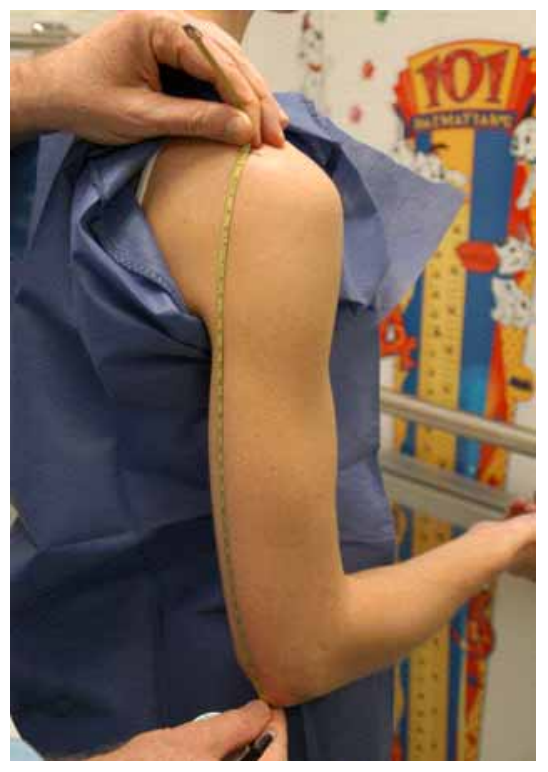

Exhibit 3-7.

Marking upper arm length midpoint

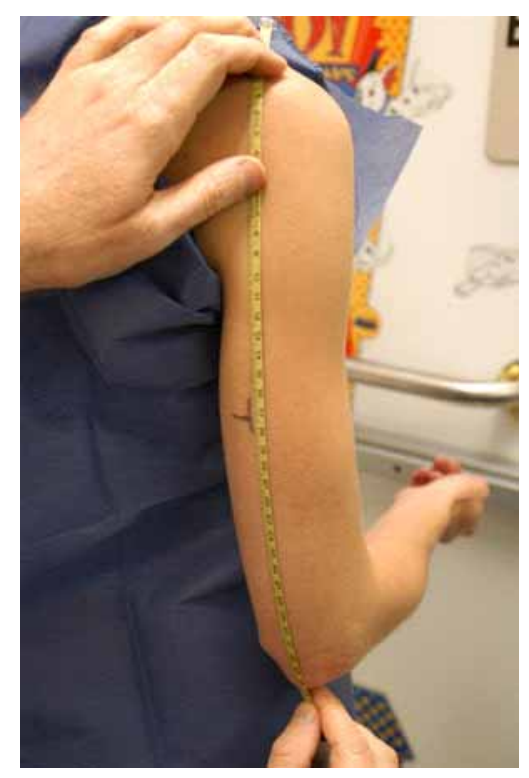


Exhibit 3-8. SP position for upper arm length and midpoint

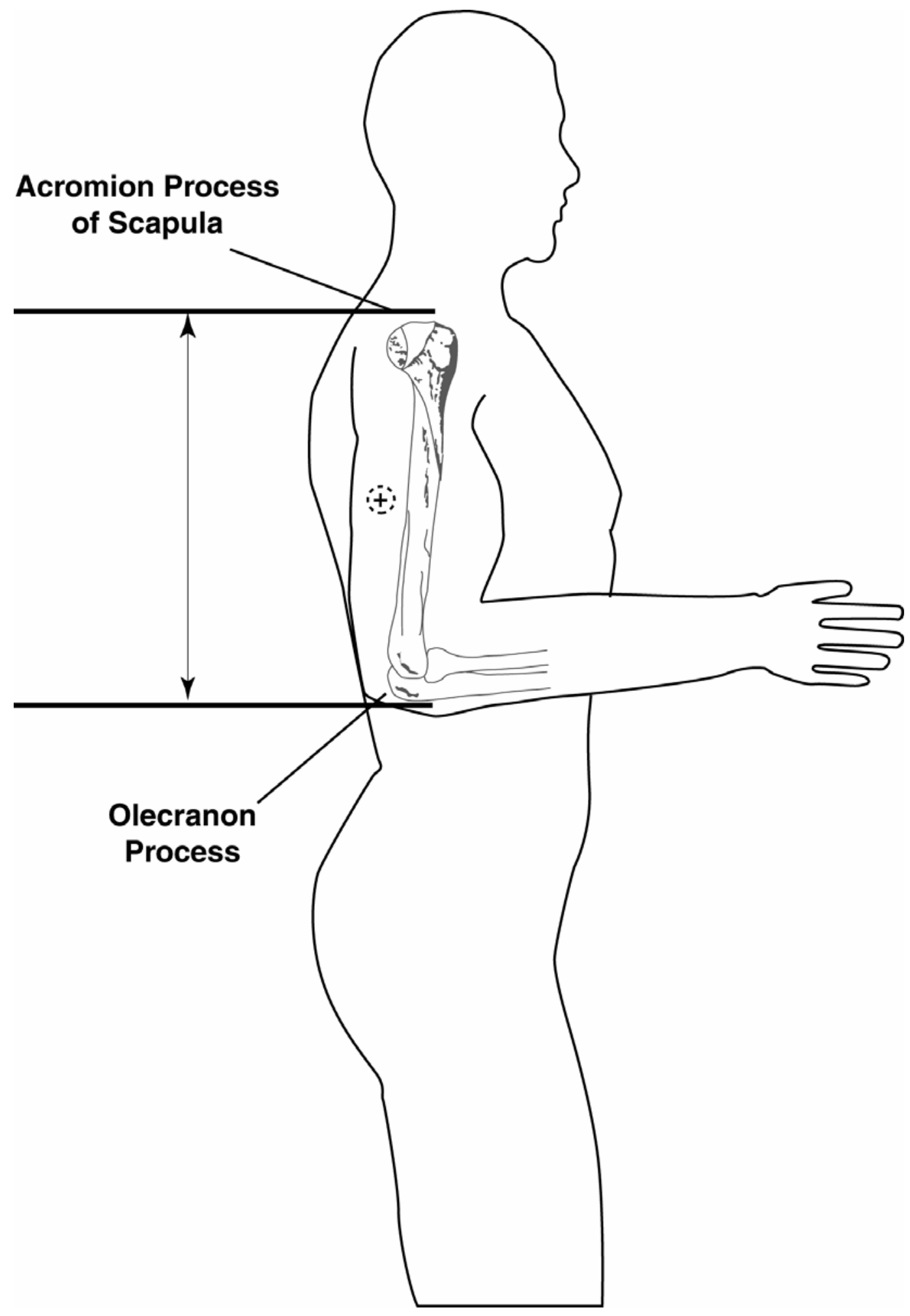




\subsection{7}

Arm Circumference

The anthropometry protocol calls for two circumference measures on participants aged 2 months and older: arm and abdominal, or waist, circumference. The arm circumference is measured on the right arm at the level of the upper arm mid-point mark. The examiner makes this mark on the posterior surface of the arm immediately after measuring the upper arm length. Thus, the procedures for making the mid-arm circumference mark are explained in Section 3.4.7, Upper Arm Length shown earlier. This section describes the procedures for measuring the arm circumference:

1. Position the SP: Ask the SP to turn so that you stand facing his or her right side. Have the participant stand upright with the weight evenly distributed on both feet, the shoulders relaxed, and the right arm hanging loosely at the sides. Flexing or tightening the arm muscles will yield an inaccurate measurement.

2. Take the measurement: Continue to stand facing the right side of the SP. Do not stand behind the SP for this measurement. Wrap the measuring tape around the arm at the level of the upper arm mid-point mark (Section 3.4.7). Position the tape perpendicular to the long axis of the upper arm. Pull the two ends of the overlapping tape together so that the zero end sits below the measurement value and the result lies on the lateral aspect of the arm (not the posterior surface). Check that the tape fits snug around the arm but does not compress the skin. Take the measurement to the nearest $0.1 \mathrm{~cm}$.

3. Record the result: Call the result to the recorder, who will enter this number on the ISIS screen. Remove the tape measure. Using the cosmetic pencil, write the arm circumference measurement on the front right part of the gown shirt of all participants aged 8 years and older. This number is used to determine the appropriate cuff size for taking the SPs blood pressure in the Physician examination. Proceed to the next circumference measure.

\subsubsection{Abdominal (Waist) Circumference}

The second circumference measure consists of the abdominal or waist circumference. Exam staff will collect the waist circumference on participants aged 2 years and older. Follow the procedures below to obtain this measure:

1. Position the SP: Instruct the participant to gather his or her gown shirt above the waist, cross the arms, and place the hands on opposite shoulders. Demonstrate the desired position of the arms. It may help to tell SPs to think of giving themselves a hug. If necessary, lower the pants and underclothing to slightly below the waist. Again, always tell the SP what you are going to do before you do it. 
2. Mark the measurement site: Stand on the participant's right side. Palpate the hip area to locate the right ilium of the pelvis. With the cosmetic pencil draw a horizontal line just above the uppermost lateral border of the right ilium. Cross this mark at the midaxillary line, which extends from the armpit down the side of the torso. Exhibit 3-9 shows the measurement site correctly marked for the waist circumference.

3. Take the measurement: Extend the measuring tape around the waist. Position the tape in a horizontal plane at the level of the measurement mark. Use the wall mirror to ensure the horizontal alignment of the tape. While the examiner remains on the SPs right side, the recorder will come around to the SPs left side to check the placement of the tape. Check that the tape sits parallel to the floor and lies snug but does not compress the skin. Always position the zero end of the tape below the section containing the measurement value. Take the measurement to the nearest $0.1 \mathrm{~cm}$ at the end of the SPs normal expiration.

4. Record the result: Call the result to the recorder, who will enter this number on the ISIS screen. Remove the tape measure. Tell the SP to let down the gown shirt and proceed to the skinfold measures.

Exhibit 3-9.

Abdominal (waist) circumference mark

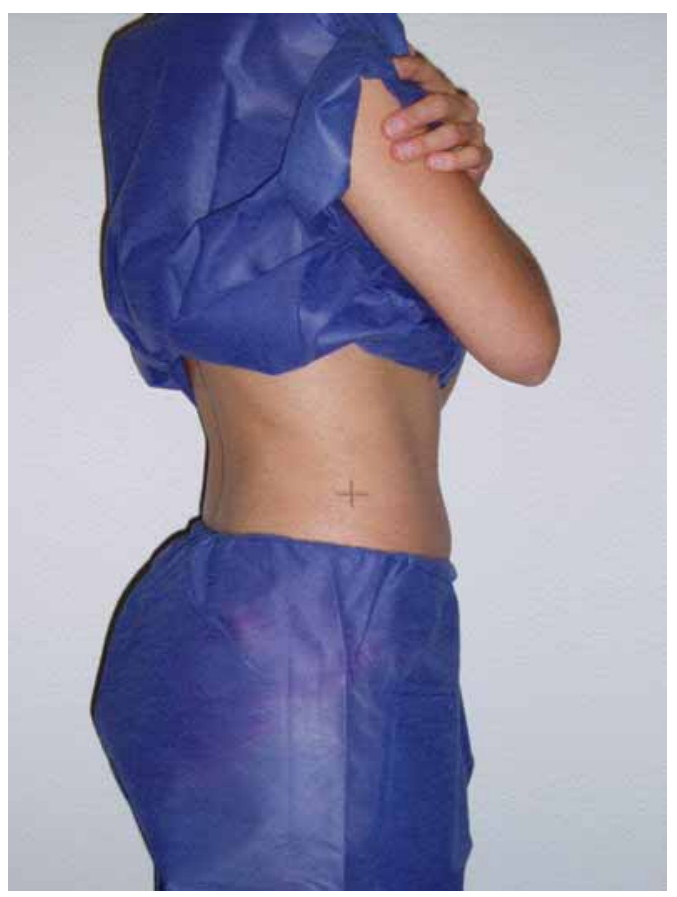

Exhibit 3-10. Measuring tape position for abdominal (waist) circumference

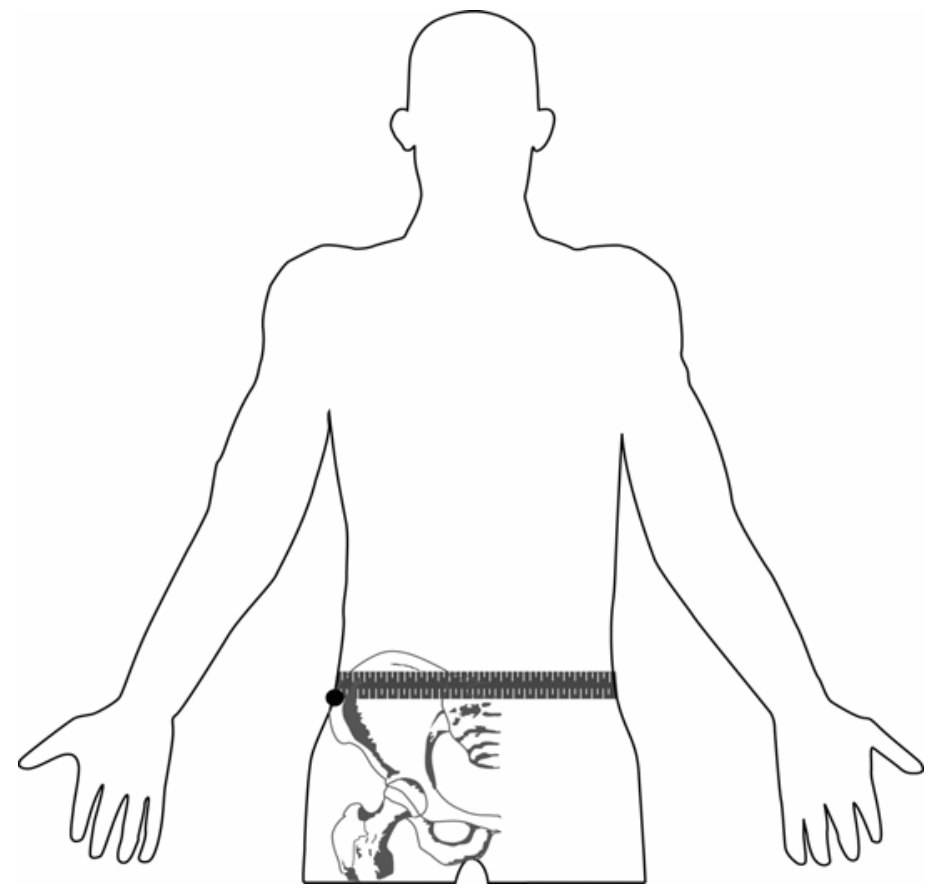




\section{Skinfolds}

The current NHANES anthropometry protocol includes triceps and subscapular skinfold measures for participants aged 2 months and older. Both types of skinfolds are measured in millimeters using the Holtain skinfold caliper.

The protocol mandates that the skinfold consist of a double thickness of skin and underlying adipose (fat) tissue. Depending on the SP the examiner may find it easier or more difficult to physically separate a fold of skin from the muscle that lies underneath. This makes skinfolds highly prone to measurement error. Do not take a skinfold measurement reading if you cannot construct a fold that has two thicknesses of skin and underlying fat. Exhibits 3-11 and 3-12 depict examples of a correct and incorrect skinfold, respectively. If you are physically unable to produce a correct skinfold with confidence call "CNO" to the recorder, who will select the Could Not Obtain comment for the corresponding measure in ISIS. In such cases, at the end of the exam the recorder should select Physical Limitation as the reason for the Partial exam status (see Section 4.10.).

\section{Exhibit 3-11. Correct skinfold measurement}

Double thickness of skin and underlying fat

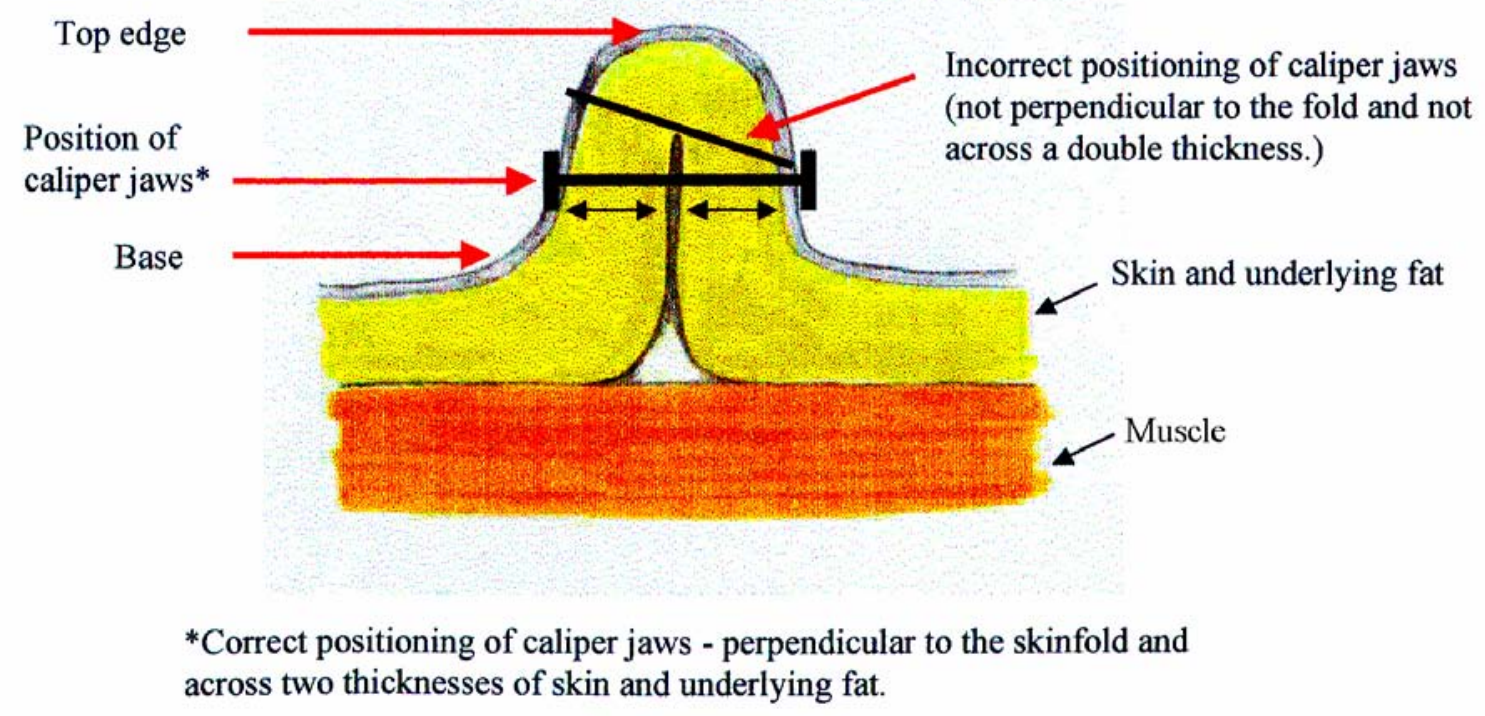


Exhibit 3-12. Incorrect skinfold measurement

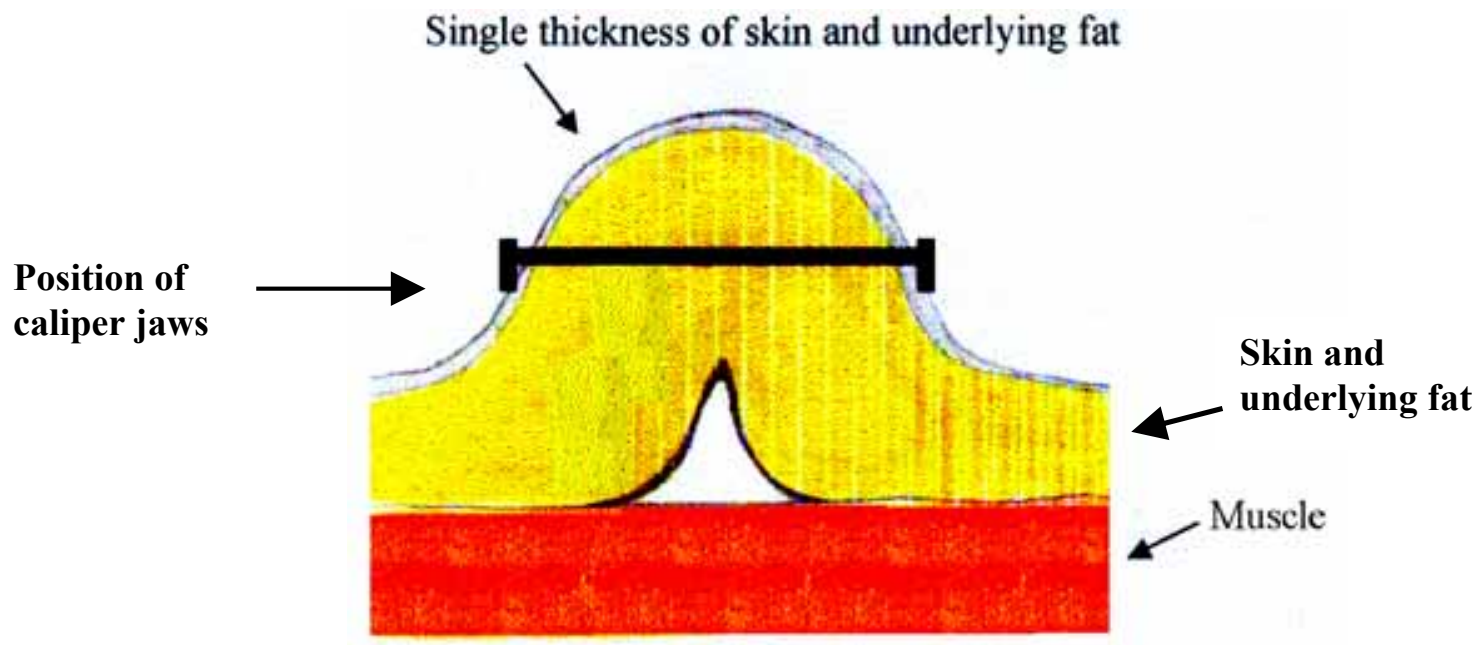

* Correct positioning of caliper jaws - perpendicular to the skinfold and across two thicknesses of skin and underlying fat.

General procedures for taking skinfold measurements are provided next. Tailored instructions for the triceps and subscapular skinfold measures will follow.

1. Prior to measuring the skinfold, carefully mark the appropriate site with a cosmetic pencil. Use the black color unless the SPs skin is dark enough to make the black mark indistinguishable. In these cases use the white pencil. Make all marks on the right side of the body.

2. The sight of the caliper may make some children frightened and anxious. In order to make them feel more relaxed, explain the procedure and demonstrate the use of the caliper on the palm of the child and/or the parent.

3. Grasp the skinfold between your thumb and index finger approximately $2.0 \mathrm{~cm}$ above the measurement mark. The amount grasped will vary depending on the thickness of the adipose tissue beneath the skin. Pull the skinfold away from the SPs body to separate the fat from the underlying muscle. The sides of the fold should be roughly parallel.

4. With your free hand, place the caliper jaws perpendicular to the length of the fold. Exhibit 3-11 contrasts the correct and incorrect positioning of the caliper jaws on the fold.

5. Continue to hold the skinfold with your thumb and forefinger. Release the handle of the calipers to apply full tension on the fold and hold this position for approximately 3 seconds. It is critical to wait roughly 3 seconds before attempting to read the skinfold 
measurement. During this time the needle on the caliper dial will settle into a final position that represents the true thickness of the fold.

6. As indicated in Section 3.3, read the caliper dial at eye level to prevent measurement error due to parallax. In other words, take the reading with your line of sight directly in front of the measurement value instead of at an angle. Measure the thickness to the nearest tenth of a millimeter $(0.1 \mathrm{~mm})$. Note that the caliper dial shows $0.2 \mathrm{~mm}$ increments. The caliper needle will commonly fall onto one of these increments. However, do not ignore the odd tenths. If after 3 seconds you observe that the needle truly lies between two lines on the dial, e.g., between $10.6 \mathrm{~mm}$ and $10.8 \mathrm{~mm}$, take the odd number in between the two, e.g., 10.7, as the measurement.

7. Before releasing the caliper and your fingers from the skinfold, call the measurement to the recorder. The Holtain calipers used in NHANES are designed to provide accurate measurements up to a maximum of $45.0 \mathrm{~mm}$. Since the face of the caliper dial shows between $0.0 \mathrm{~mm}$ and $40.0 \mathrm{~mm}$, in order to measure above $40.0 \mathrm{~mm}$ the needle will need to run a full circle and past the 0.0. However, since measurements over 45.0 will be considered invalid, ISIS will not accept entries above this number for quality control purposes. Therefore, record skinfold measurement results greater than $45.0 \mathrm{~mm}$ as EC (Exceeds Capacity) beside the appropriate ISIS field. See also Section 4.7.

8. Finally, after calling the measurement result to the recorder, remove the caliper jaws then let go of the fold. Perform all skinfold measures according to the above procedures. When finished hand the calipers to the recorder to put away until the next exam.

\subsubsection{Triceps Skinfold}

The triceps skinfold is measured at the upper arm mid-point mark on the posterior surface of the right upper arm. The procedures for making this mark are explained in Section 3.4.7, Upper Arm Length. Follow the procedures below to perform the triceps skinfold measure:

1. Position the SP: Ask the SP to turn so that you stand behind his or her right side. Have the participant stand upright with the weight evenly distributed on both feet, the shoulders relaxed, and the arms hanging loosely at the sides. Flexing or tightening the arm muscles will yield an inaccurate measurement.

2. Grasp the skinfold: Using your thumb and index finger, grasp a fold of skin and subcutaneous adipose tissue approximately $2.0 \mathrm{~cm}$ above the mid-arm circumference mark. If you have difficulty separating the skinfold from the triceps muscle, start at the elbow where the tissue tends to be looser and work your way up to the mark. Ensure that the skinfold consists of a double thickness and sits parallel to the long axis of the arm. 
3. Position the caliper: Holding the skinfold $2.0 \mathrm{~cm}$ above the circumference mark, place the tips of the caliper jaws over the complete skinfold. Ensure that the mark remains centered between the tips and that the jaws sit perpendicular to the length of the skinfold. Exhibit 3-13 shows the correct placement of the caliper for this measurement.

4. Take the measurement: Continue to hold the skinfold in place and release the caliper handle to exert full tension on the skinfold. Wait 3 seconds for the needle on the caliper dial to settle on an accurate measurement. Read the thickness to the nearest 0.1 $\mathrm{mm}$.

5. 5. Record the result: Call this result to the recorder, who will enter this number on the ISIS screen. Remove the caliper jaws then let go of the skinfold. Proceed to the next measure, the subscapular skinfold.

Exhibit 3-13. Triceps skinfold measurement

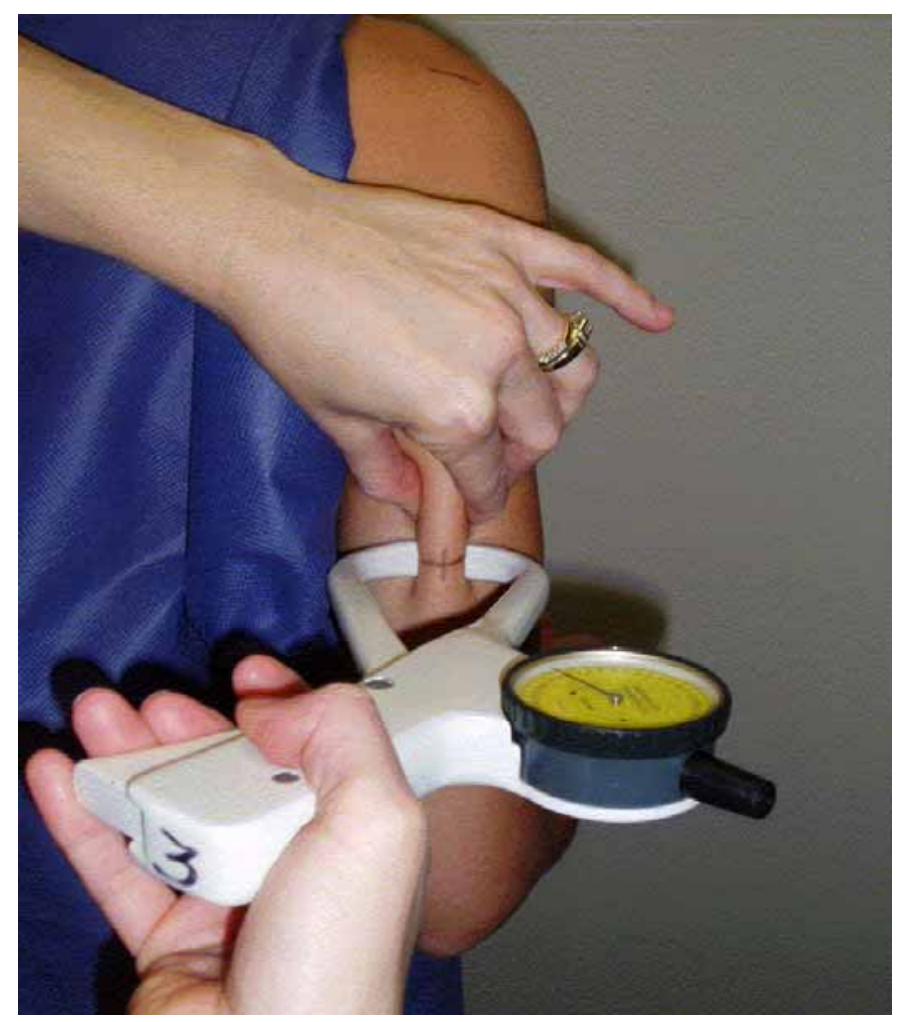




\subsubsection{Subscapular Skinfold}

The subscapular skinfold is measured at the inferior angle of the right scapula. For efficiency make the mark needed for this measure immediately following the upper leg length measure (Section 3.4.6). Instructions for performing the subscapular skinfold measure are provided below:

1. Position the SP: Similar to the triceps skinfold measure, turn the SP so that you stand behind his or her right side. Have the participant stand upright with the weight evenly distributed on both feet, the shoulders relaxed, and the arms hanging loosely at the sides.

2. Mark the measurement site: Tell the SP that you are going open the gown in the back and mark the skin with the cosmetic pencil. Open the back of the gown and palpate for the inferior angle, or triangle portion, of the right scapula (Exhibit 3-14). Using the cosmetic pencil, mark a cross $(+)$ on the inferior angle (Exhibit 3-15). Make the first line at 45 degrees to the spine and cross this with a line that bisects the inferior angle of the scapula.

3. Grasp the skinfold: Using your thumb and index finger, grasp a fold so that the index finger remains situated roughly $2.0 \mathrm{~cm}$ above and medial to the inferior angle of the scapula. Due to tightness in this area of the back on many SPs, obtaining this skinfold measure can be a challenge. In these cases where you experience difficulty separating the subscapular skinfold from the underlying tissue, begin grasping the fold with the thumb and index finger spread wide.

4. Position the caliper: Continue to hold the skinfold in place. With the other hand, set the top jaw of the caliper on the "+" mark. This differs from the triceps skinfold procedure in which the mark is centered between the caliper tips. Position the tips of the caliper jaws over the complete skinfold perpendicular to the length of the fold and roughly $2.0 \mathrm{~cm}$ lateral to the fingers (Exhibit 3-16).

5. Take the measurement: Continue to hold the skinfold in place and release the caliper handle to exert full tension on the skinfold. Wait 3 seconds for the needle on the caliper dial to settle on an accurate measurement. Read the thickness to the nearest 0.1 $\mathrm{mm}$.

6. Record the result: Call this number to the recorder, who will enter this number on the ISIS screen. Remove the caliper jaws then let go of the skinfold. The subscapular skinfold constitutes the final anthropometric measure for the exam. 
Exhibit 3-14. Location of subscapular skinfold

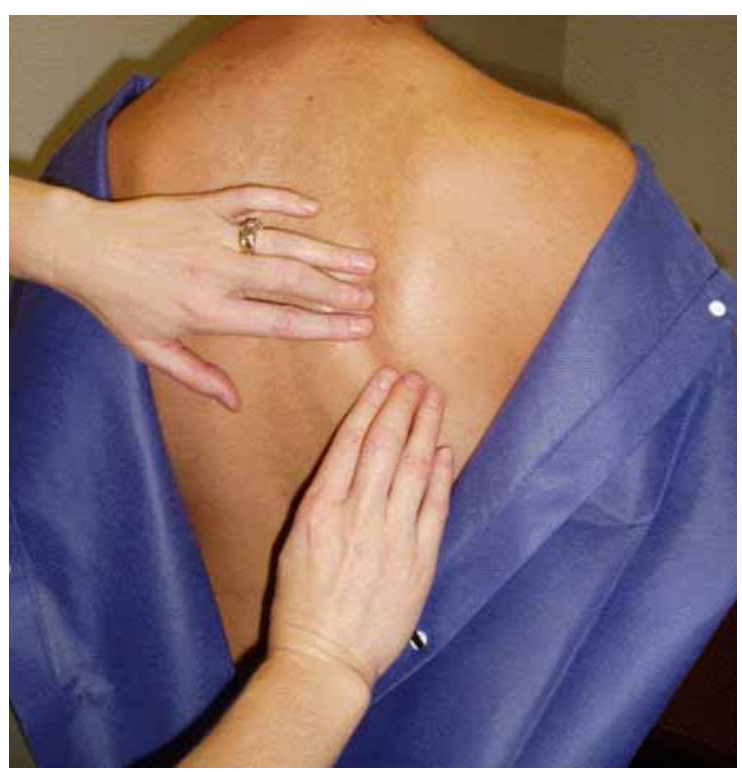

Exhibit 3-15. Subscapular skinfold mark

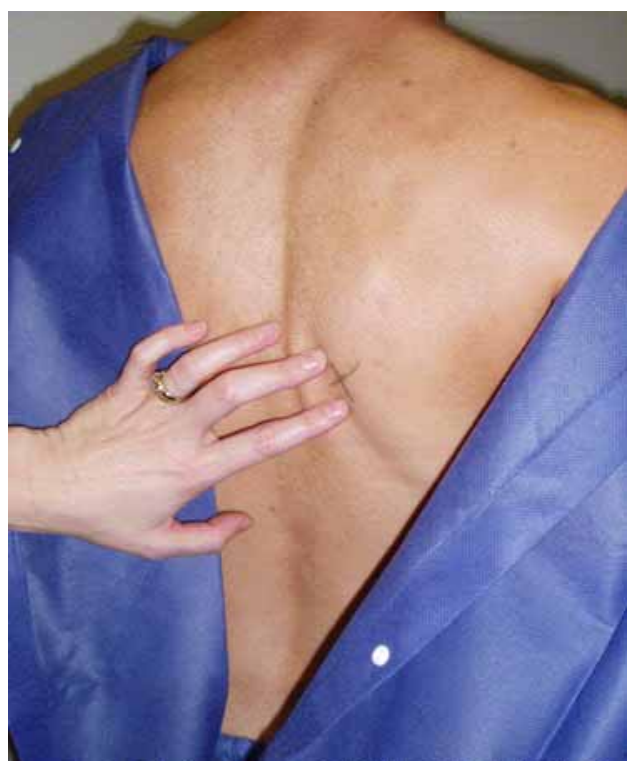

Exhibit 3-16. Subscapular skinfold measurement

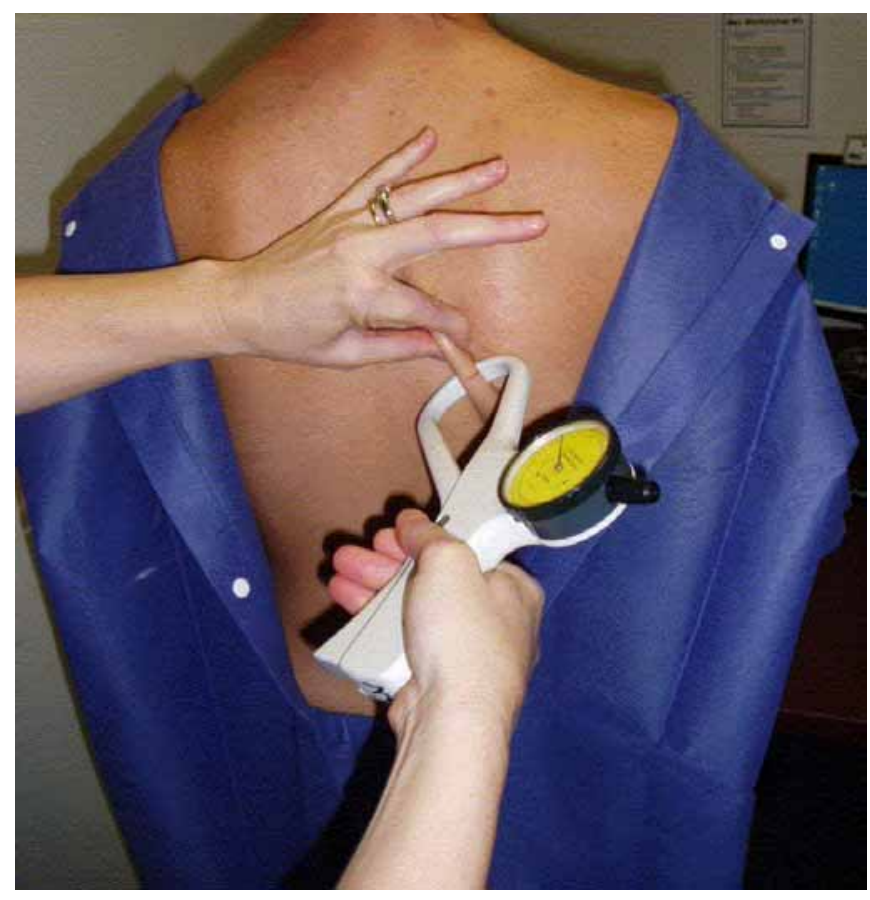




\section{5}

\section{Special Considerations}

Obtaining complete and accurate body measurement data can be more challenging under some circumstances. The following situations may require certain modifications to the procedures that would be followed under routine conditions.

\subsubsection{SPs in Street Clothes}

Some SPs refuse to change into the shirt and/or pants provided to them as part of the standard survey examination gown. If a participant enters the exam room wearing his or her street clothes, complete as many anthropometric measurements as possible. For example, the weight and height measures can easily be obtained. Exam staff can also collect the upper arm length, arm circumference, and triceps skinfold measurements if the SP has a loose fitting or sleeveless shirt. For infants, ask the parent or guardian to undress the child leaving only the diaper and t-shirt.

Perform the weight measurement procedures described in Section 3.4.1 with the following caveats:

- Shoes - Ask the SP to remove his or her shoes before stepping on the scale. If the SP refuses to remove the shoes in order to step on the scale, code Could Not Obtain (CNO) for the weight measure in ISIS. Weight data collected with the SP wearing shoes on the scale will be considered invalid.

- $\quad \mathbf{L}$ - On the ISIS screen select Clothes (CL) from the Comments box beside the Weight field. This code indicates that the SP wore street clothes while standing on the scale. Do not use the CL code for infants wearing diapers or a t-shirt.

- MA - If you successfully obtained the weight but the SP wore a medical appliance that could not be removed, such as a cast or prosthesis, add the Medical Appliance (MA) code from the ISIS Comments box. 


\subsection{2}

\section{Children}

The procedures for performing anthropometric measures on children must take into account their smaller size as well as extra safety precautions:

- Weight - When an infant or toddler SP cannot stand unassisted on the digital weight scale, collect the weight following the Tare procedures described in Section 4.2.2. In ISIS be sure to select the Clothes (CL) code beside the weight field for a child wearing street clothes but not for an infant wearing a diaper and t-shirt.

- Recumbent length - As mentioned in Section 6.2, exam staff must carefully hold small children on the infantometer at all times because they can quickly flip themselves over or easily fall. Move the screen cursor to the GET button before positioning the child, in order to capture the measurement immediately after the child assumes the correct position.

- Upper Arm Length and Circumferences - For toddlers and small children up to roughly 6 or 7 years of age, the examiner may opt to stand the child on the white sitting box. In this position the examiner can better control the child's movements and take measurements at eye level for data accuracy. Ensure that the box sits against the wall and ask the child to hold the safety bar for balance. If the child cannot stand alone on the box, take the measurements with the child sitting in the parent's lap. Ask parents to place infants over their left shoulder.

- Skinfolds - The examiner should also stand small children on the white sitting box for skinfold measurements if this allows measurements to be taken at eye level. Children who are unable to stand on the box should sit on the parent's lap or be placed

over the shoulder in the case of infants. Do not let the calipers become the object of the child's attention. Rather, hold them behind your body until you are ready to take the measure. Demonstrate the use of the caliper on the palm of the child and/or the parent. This often helps make the child and parent feel more comfortable with the skinfold measurement procedures.

\subsubsection{SPs in Wheelchairs}

Provided the SP is willing and able to participate, exam staff should attempt to collect the following subset of anthropometric measurements on SPs who are in wheelchairs:

- Upper Arm Length

- Arm Circumference

- Triceps skinfold 
To prepare the SP for these measurements, direct him or her to the right side of the wheelchair so that the arm of the chair does not restrict the right arm. Ensure that the SP can extend the right arm without interference from the chair. As with ambulatory participants, DO NOT try to lift the SP or bear his or her weight in order to facilitate the examination protocol.

\subsubsection{Amputees}

For SPs who have any part of a limb on the right side amputated, collect the required measurement data on the SP's left side. For example, if the SP has part of his or her right arm missing, obtain the Upper Arm Length measurement and mark the midpoint on the left arm. Measure the Arm Circumference and Triceps Skinfold on the left arm as well. Enter CNO in the Comment box only if both arms are amputated or for some other reason you cannot obtain these three measurements.

During the exam, carefully observe whether the SP has any amputations. For data quality purposes it is important to take amputations into account, for example in relation to the measured weight. The anthropometry protocol does not require the examiner or recorder to ask the SP outright if he or she has any amputations. Amputations may be obvious on many SPs, especially amputations of the arms and the leg that is measured as part of the exam. However, if the exam staff has doubts or difficulty determining this information based on observation alone, then ask the SP directly: "Do you have any amputations?"

Record all amputation-related information on the ISIS Amputations screen (Section 4.9) prior to finishing the exam. Specifically, ISIS will ask you to record whether or not the SP has any amputations. If so, ISIS will prompt you to record more details: the particular extremity or extremities affected, i.e., upper or lower, right or left; and where the amputation lies, i.e., above or below the elbow or knee.

\subsubsection{Comprehension or Language Difficulties}

Some SPs may have difficulty understanding the examination instructions. Exam staff should use extreme caution when attempting to conduct the anthropometry component on these participants. If the SP cannot understand your instructions due to a developmental disability or other type 
of physical or mental impairment, ask if a family member or friend accompanied the SP to the MEC who can help explain your directions to the SP. Regardless, if you believe the SP cannot comprehend well enough for you to safely and accurately carry out the examination protocol, then on the ISIS screen code the appropriate measure(s) as Could Not Obtain (CNO) and select "Communication Problem" as the reason for the Partial or Not Done exam status.

For many SPs language barriers are a common cause of difficulty in understanding examination instructions. For Spanish-speaking SPs, if the examiner is not English-Spanish bilingual, a Spanish interpreter will be assigned to interpret for the SP and the examiner during the exam. In most cases the coordinator will assign a bilingual recorder to conduct the component with the examiner. If the SP speaks a language other than English or Spanish, arrangements will be made ahead of the anthropometry exam to identify an appropriate interpreter. Regardless, as in the case of other forms of comprehension difficulties, if you cannot safely and accurately perform the exam on the SP, then code the affected measure(s) as Could Not Obtain (CNO) on the ISIS screen and select "Communication Problem" as the reason for the Partial or Not Done exam status. See the NHANES Interpretation Guidelines for procedures related to the interpretation of MEC examination components and working with interpreters.

\subsection{Post-examination Procedures}

At the end of the examination, remove any remaining cosmetic pencil marks from the SPs skin with a few drops of baby oil on a piece of gauze. Tape the SPs pant leg and sleeve where they were cut during the exam. The recorder will hand the gauze pads with baby oil and strips of masking tape to the examiner; return the SPs jewelry, wallet, purse, or other belongings that may have been set aside; and put away equipment and supplies in preparation for the next examination.

To end the component, the recorder will click the Finish button on the ISIS screen. This signals the coordinator to make the next assignment for that SP. Do not click Finish until the SP is completely ready to be escorted out of the room. Wait for a message from the coordinator indicating where to direct the SP. Thank the SP for participating and accompany him or her to the next exam room or to the reception area. 


\section{ISIS DATA ENTRY}

\subsection{General Screen Information}

When the coordinator assigns an SP to the Anthropometry or Body Measures room, a communication dialog box will appear on the ISIS computer screen. This will inform you that an SP has been assigned to this component. Click the Close button to remove the dialog box from the screen.

To begin the examination, click the "Logon SP" icon, the first icon on the left in the Standard toolbar. ISIS will present a dialog box that asks for the name and password of the examiner and the recorder. Wand the bar code on the SP's identification bracelet or type the SP's ID number to log the SP into the component. This will activate a dialog box containing descriptive information about the SP (i.e., name, SP ID, age, etc.). Verify that the correct participant name appears on the screen. Contact the coordinator and MEC manager for assistance if the information in ISIS appears incorrect. Otherwise, click OK to initiate the examination.

Exhibit 4-1. Logon SP screen

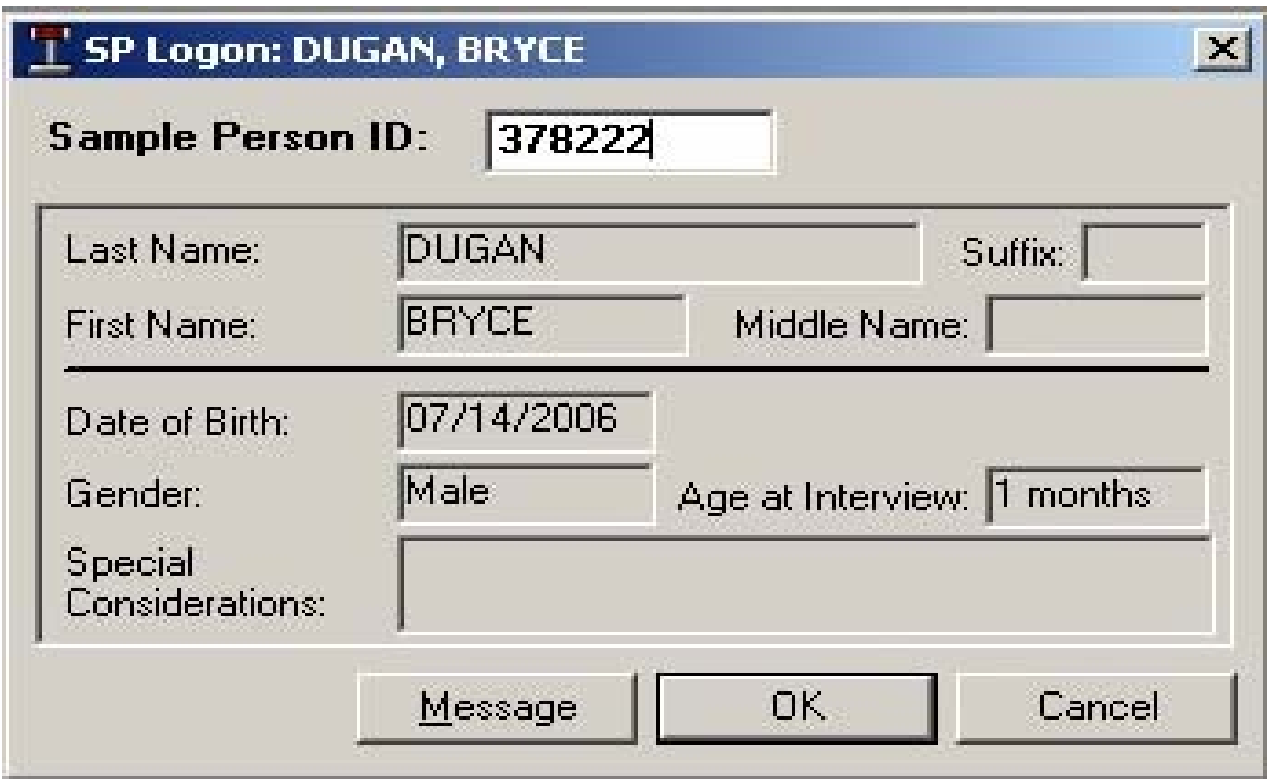


All ISIS screens have similar characteristics. As shown below (Exhibit 4-2), at the very top of the screen is a Title bar containing the component name (Anthropometry Subsystem), Stand number, Session number, and Session date and time. Below this are the Menu bar and Standard toolbar icons, which provide software application commands and shortcuts. Under the standard toolbar sits a second title bar that identifies the examination component (Body Measures), Stand number, Session number, and Session date and time. Below the second title bar is the SP ID, name, age, gender, and current date and time. The component screen name lies in the upper left of the main window area.

Exhibit 4-2. ISIS screen characteristics

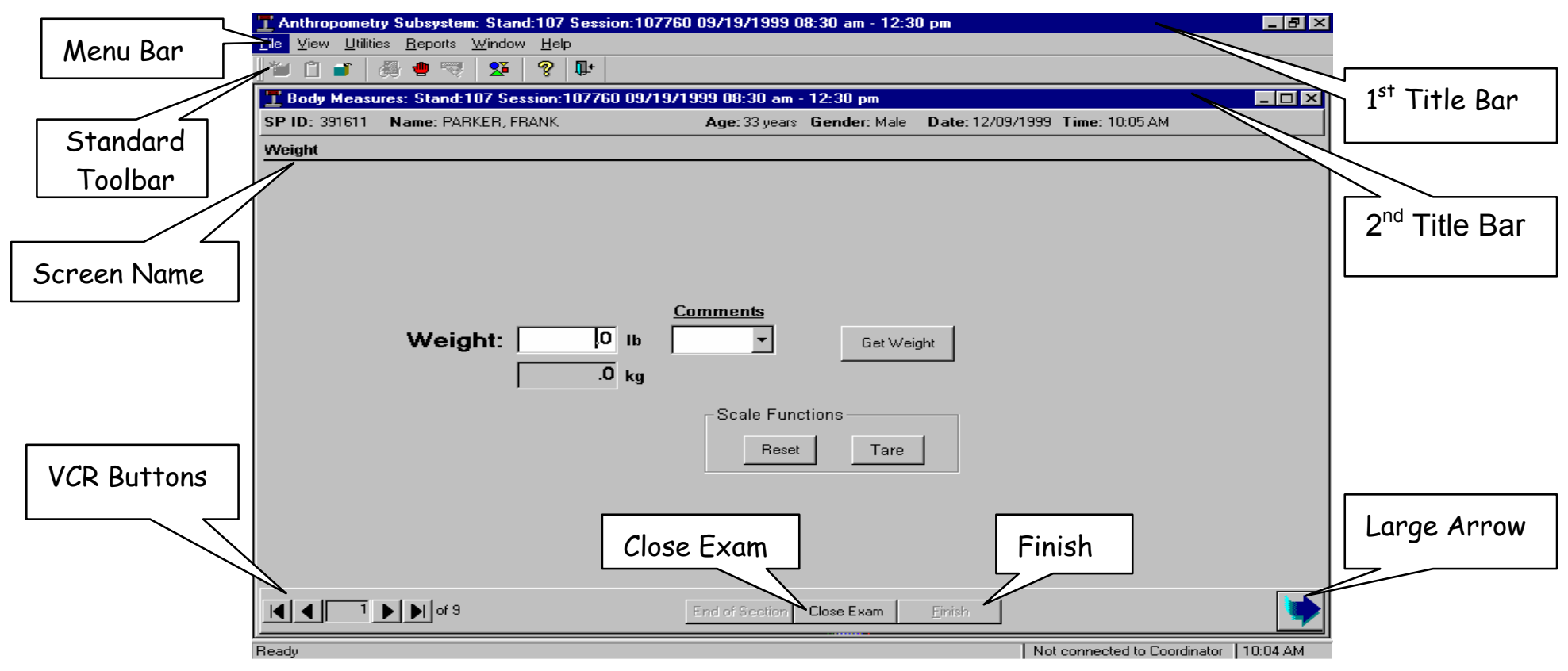

At the bottom left corner of the screen is the ISIS screen number and a set of arrow buttons for navigating the screens: The far left button moves to the first screen and the far right button moves to the last screen. Directly beside the screen number, the left button moves to the previous screen and the right button moves to the next screen. At the bottom right corner of the screen is a large arrow. Always click on the large arrow to advance to the next screen. 
In the middle of the bottom of the screen are two buttons: Close Exam and Finish. Clicking Close Exam will delete any data captured on the current screen, terminate the exam, and code the exam status as Partial or Not Done. NEVER click the Close Exam button unless the exam must be discontinued and there is no other appropriate means to exit the application. Click the Finish button at the end of an exam. This sends a signal to the coordinator that the SP is available for the next assignment.

As you proceed through the examination, certain ISIS entries will activate an Edit Check box (Exhibit 4-3). This window appears if the recorder enters a number that ISIS determines to be out of the acceptable range. Specifically, ISIS identifies numeric data entries as out of range as those that are less than the $1^{\text {st }}$ percentile or greater than the $99^{\text {th }}$ percentile for the SP's age and gender. In these cases, the Edit Check box will present the message, "Check that measure." The recorder must read the edit check message aloud to prompt the examiner to review the measurement for accuracy.

Exhibit 4-3. Edit check box

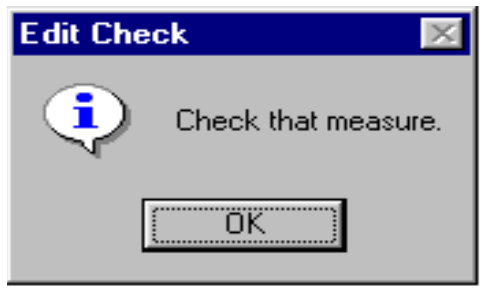


The first data entry screen is the Weight screen (Exhibit 4-4) Weight in kilograms is collected on all SPs.

\section{Exhibit 4-4. Weight screen}

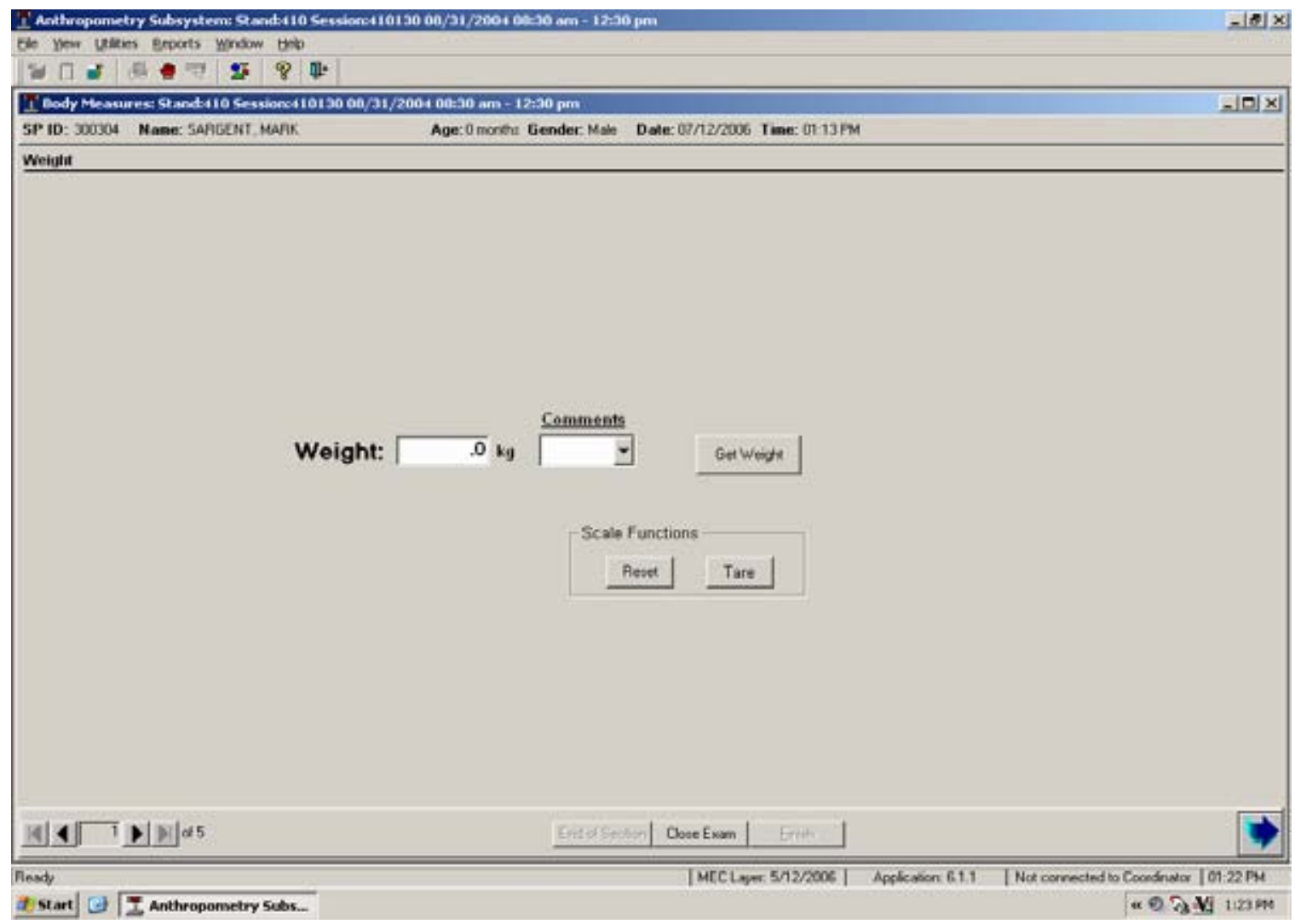

When you open this screen the cursor will automatically appear in the Weight field. After the SP steps on the digital weight scale and the weight appears on the measurement device, click the "Get Weight" button on the screen. ISIS will capture the SP's weight directly from the scale and display the number in the Weight field.

If the digital weight scale was set to pounds (lb) instead of kilograms (kg), when you click "Get Weight," ISIS will present a warning message asking you to change the display to kg and click Get Weight again (Exhibit 4-5). In this case click OK to the warning message, change the scale setting to $\mathrm{kg}$, and retry capturing the weight into ISIS. 
Exhibit 4-5. Weight screen - lb kg warning message

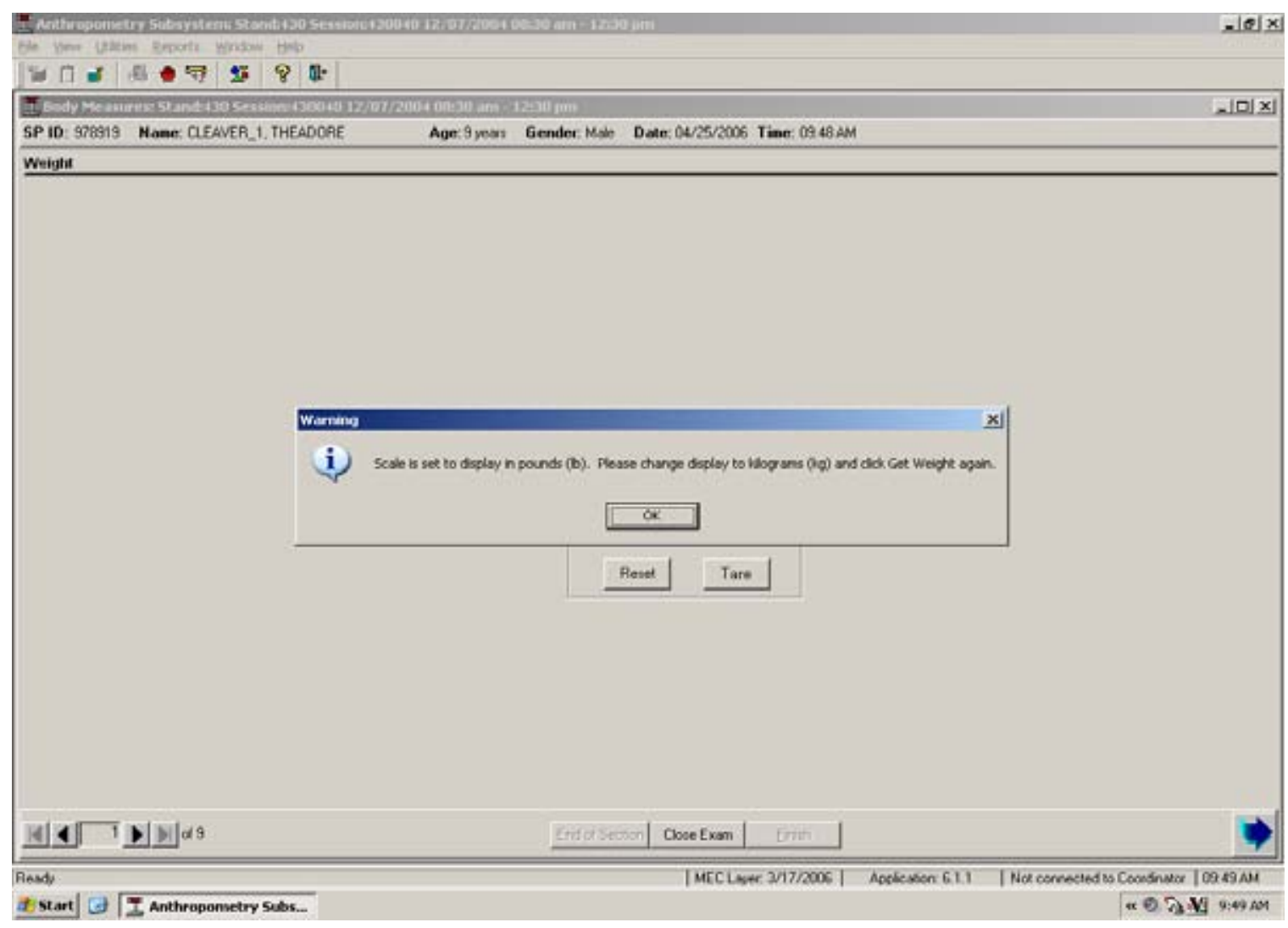

\subsubsection{Weight Comments}

Beside the Weight field, a Comments box provides the following codes in a drop-down list: $\mathrm{EC}, \mathrm{CNO}, \mathrm{CL}$, and MA (Exhibit 4-6).

- $\quad$ EC = Exceeds Capacity: Select EC if you cannot capture or manually enter the weight due to: (1) the SP exceeds the capacity of the digital weight scale; and (2) the SP exceeds the capacity of two portable scales when standing with one foot on each scale.

- $\quad$ CNO = Could Not Obtain: Select CNO if you cannot obtain the weight for a reason other than EC.

- $\quad \mathbf{C L}=$ Clothing: Select CL if you successfully obtained the weight but the SP wore street clothes instead of the standard MEC shirt and pant gown. Do not select CL for infants wearing diapers or a t-shirt. 
NOTE: If the SP wears shoes to the exam, ask him or her to remove them before stepping on the scale. If the SP refuses to remove the shoes in order to step on the scale, code the weight as CNO. Weight data collected with the SP wearing shoes on the scale will be considered invalid.

- $\quad$ MA = Medical Appliance: Select MA if you successfully obtained the weight but the SP wore a medical appliance that could not be removed, such as a cast or prosthesis.

Exhibit 4-6. Weight screen - comments list

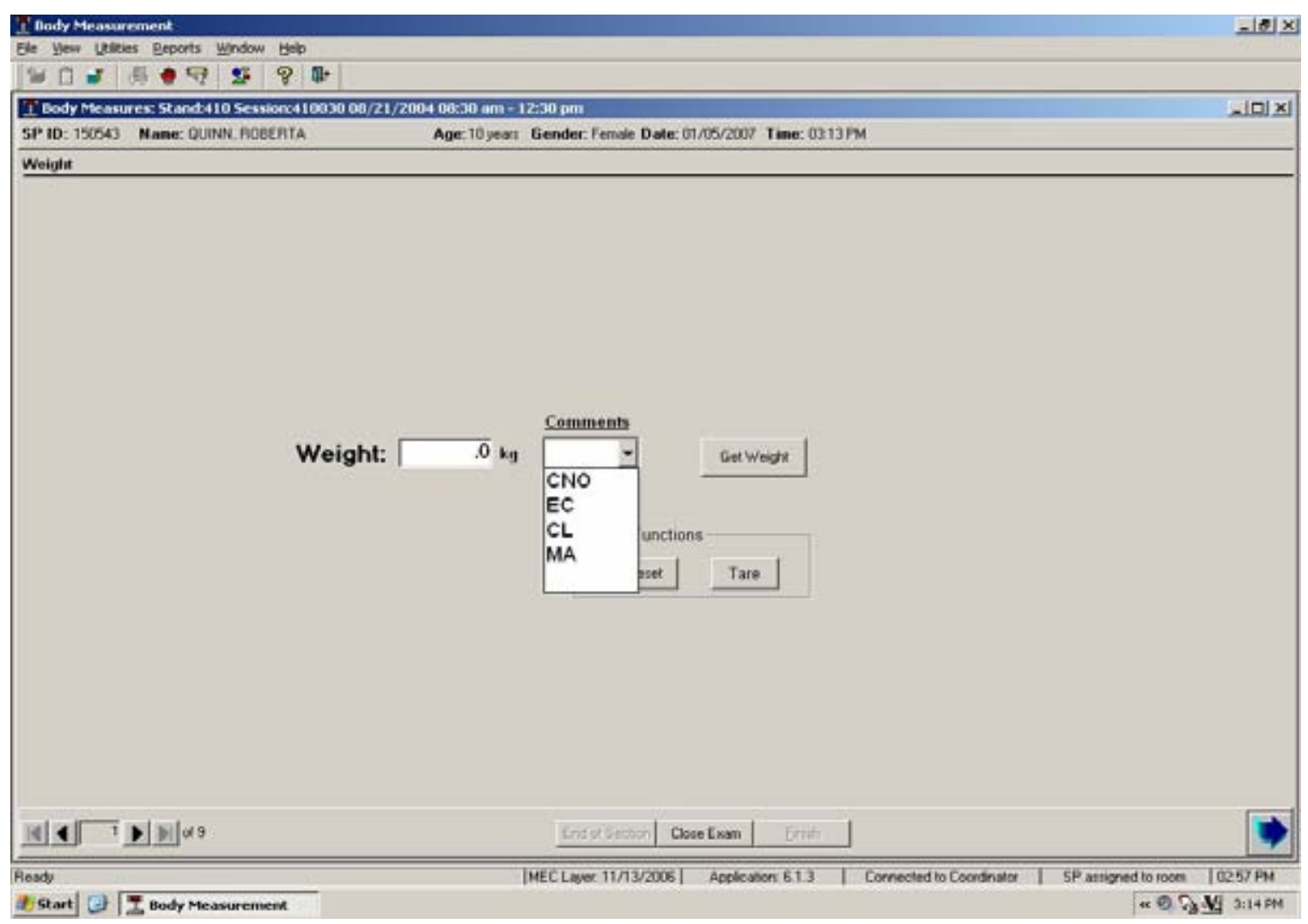

4.2.2

Tare and Reset

Use the Tare and Reset scale functions on the Weight screen when an infant or toddler SP cannot stand unassisted on the digital weight scale. In these cases an adult will assist in weighing the child. First, either the child's guardian or the examiner will stand alone on the scale while the recorder clicks the Tare button on the screen. This sets the reading on the scale measurement device to zero. Next, the child is handed to the person on the scale. The measurement device will now read only the weight of the child. As soon as the scale measures this weight the recorder will click "Get Weight" to transfer the 
number to ISIS. After the child's weight is successfully captured on the screen, the recorder will click "Reset" to undo the tare function.

\subsection{Stature Screen}

The second screen in the ISIS anthropometry application is the Stature screen (Exhibit 4-7a-d). In accordance with the anthropometry protocol, the screen will reflect different measurement fields depending on the age of the SP:

- SPs aged 0-6 months: Recumbent Length and Head Circumference

- SPs aged 7-23 months: Recumbent Length

- SPs aged 24-47 months: Recumbent Length and Standing Height

- SPs aged 4 years and older: Standing Height

Click the "Get" button to transfer the recumbent length and standing height measurement directly from the infantometer and stadiometer, respectively, to ISIS. These measurements may also be manually entered. The head circumference measurement can only be entered manually. After each measure is successfully captured in ISIS, a computer-generated voice will repeat the number aloud. Always verify that this is the correct number. 
Exhibit 4-7a. Stature screen - SPs 0-6 mos

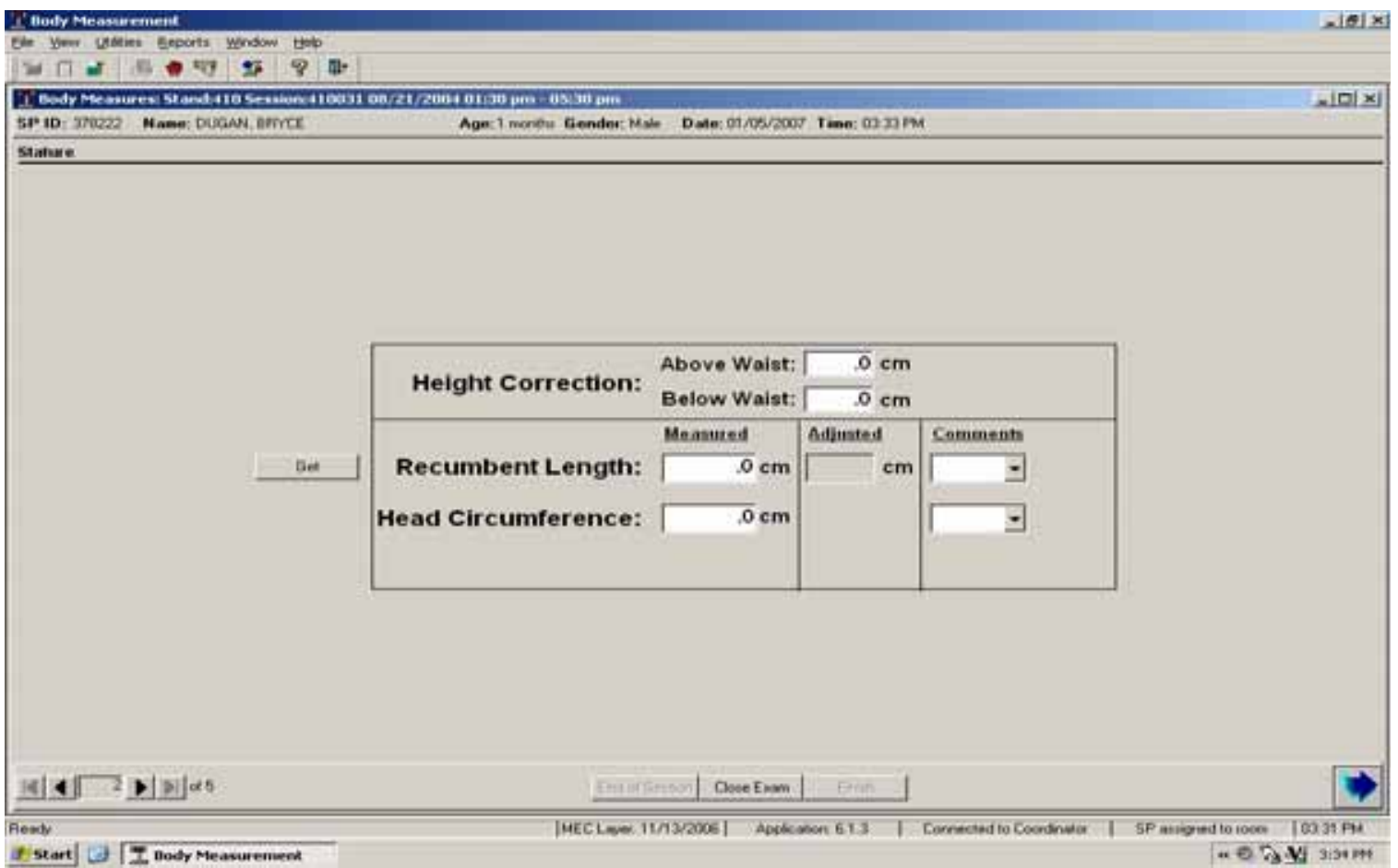

Exhibit 4-7b. Stature screen - SPs 7-23 mos

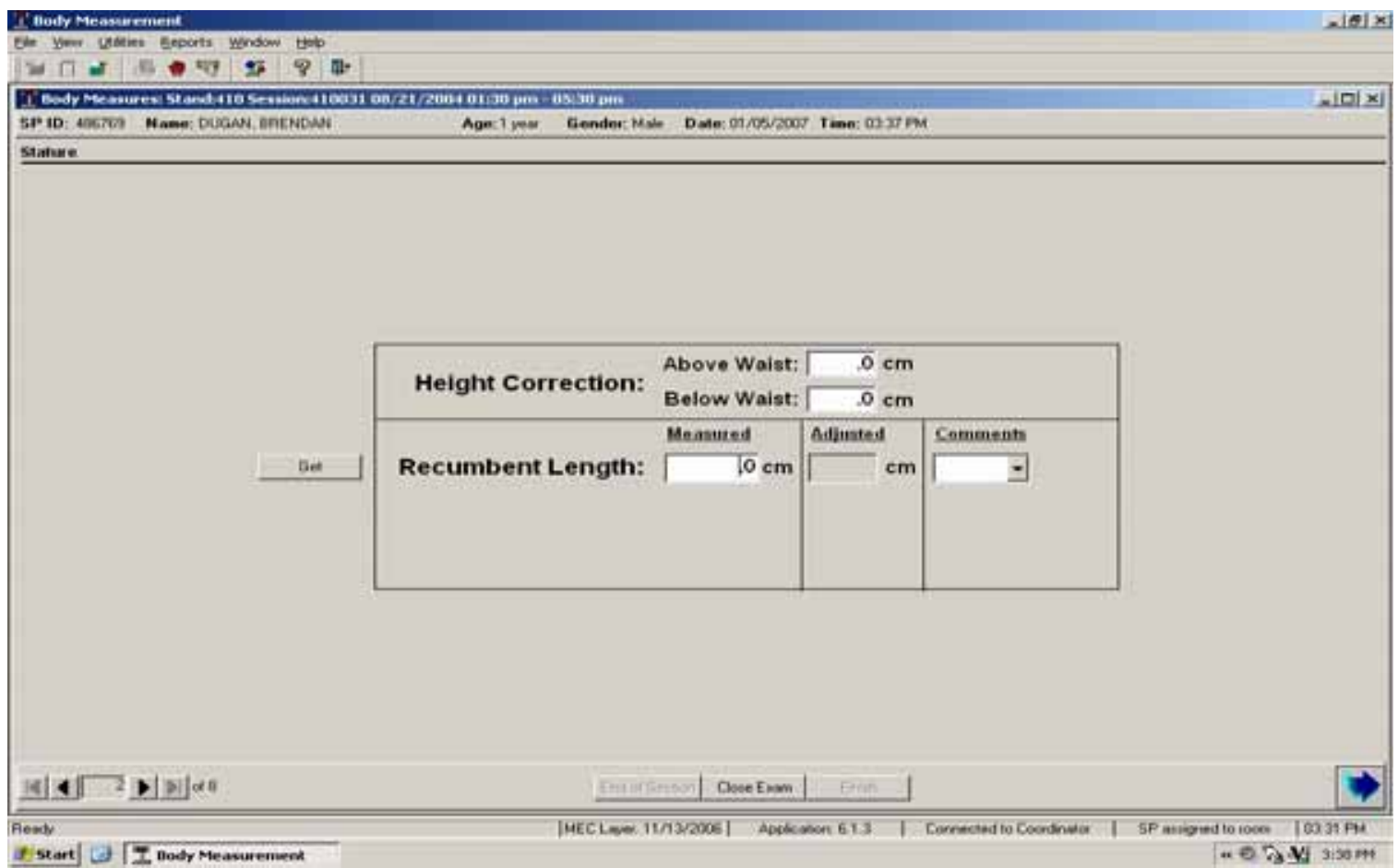


Exhibit 4-7c. Stature screen - SPs 24-47mos

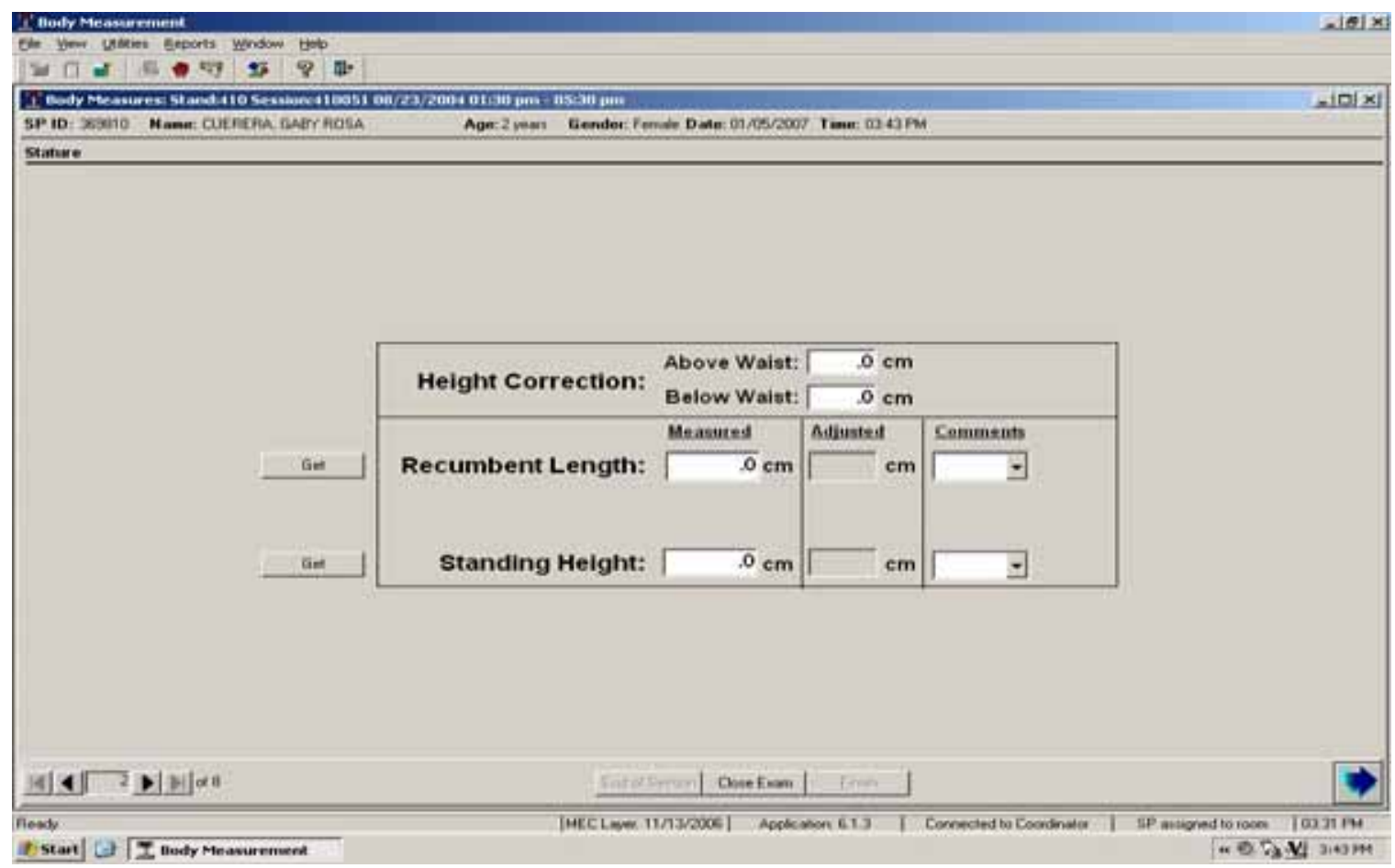

Exhibit 4-7d. Stature screen - SPs 4yrs+

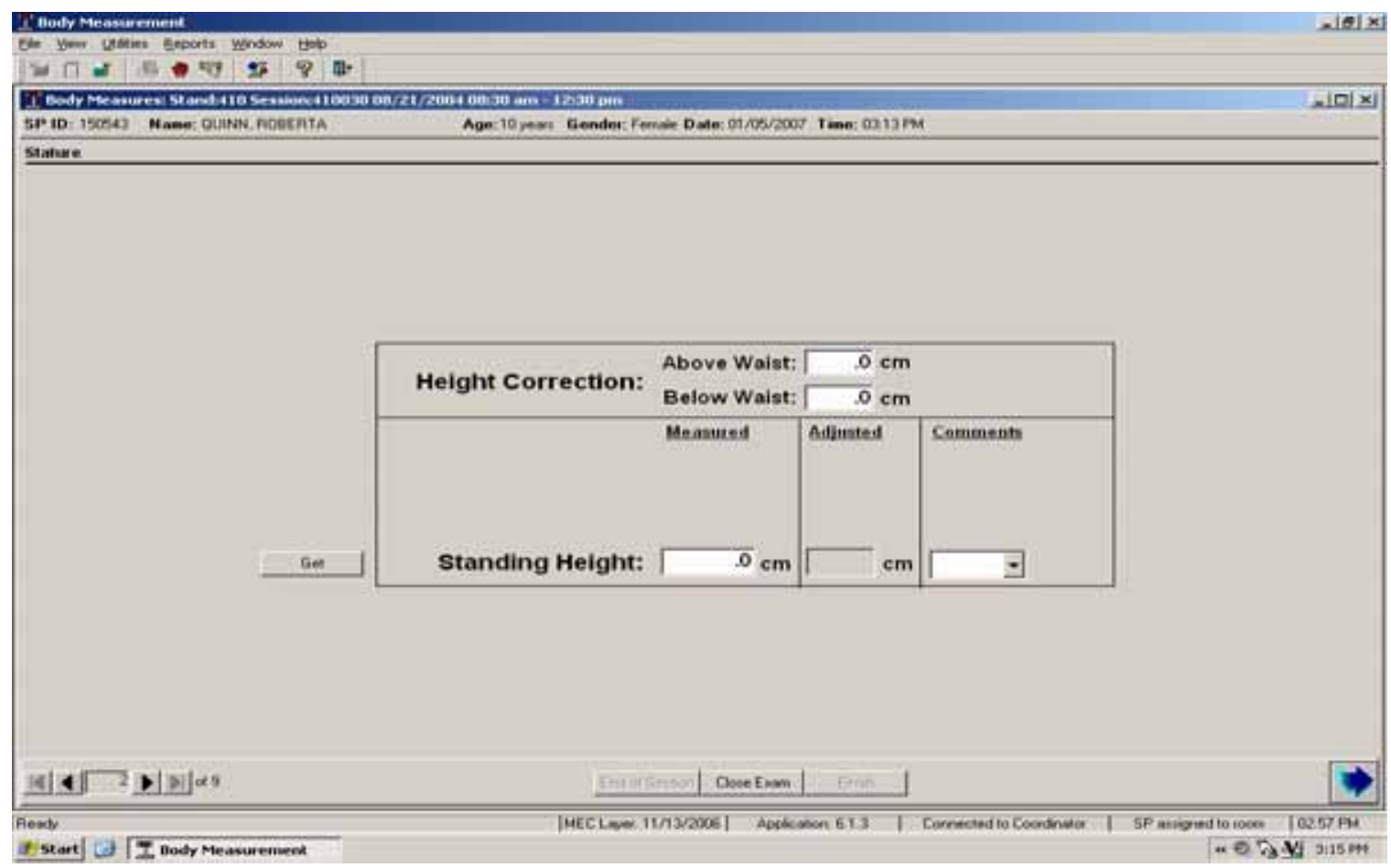




\subsubsection{Height Correction}

The top portion of the Stature screen offers two fields for Height Correction: Above Waist and Below Waist (Exhibits 4-8a and b). Using the designated height adjustment ruler, the exam staff will make corrections to the SPs height in two cases:

- Above Waist - The SP refuses (or is unable) to remove a hairpiece such as a barrette, bun, or braids that would otherwise prevent an accurate stature measurement. The technologist measures the hairpiece with the ruler and the recorder enters this number in the Height Correction: Above Waist field.

- Below Waist - The SP refuses (or is unable) to remove his or her shoes for the stature measurement. The technologist measures the height of the shoe heel with the ruler and the recorder enters this number in the Height Correction: Below Waist field.

The Stature screen (Exhibits 4-8a and b) also displays two columns associated with corrections to the recumbent length and standing height measurements:

- Measured - The uncorrected height measurement captured or entered into ISIS from the infantometer or stadiometer.

- Adjusted - The adjusted height measurement, taking into account the height correction. ISIS automatically calculates the adjusted height by subtracting the height correction number entered in the Above Waist and/or Below Waist field from the uncorrected height measurement value.

\subsubsection{Stature Comments}

Beside the Measured and Adjusted columns is a Comments column for specific notes regarding recumbent length and standing height measurements. The Comment box provides the following codes in a drop-down list: EC, CNO, and NS.

- $\quad$ EC = Exceeds Capacity: Select EC if you cannot capture or manually enter the height because the SP is too tall or long to be measured by the stadiometer or infantometer, respectively. 
- $\quad$ CNO $=$ Could Not Obtain: Select CNO if you cannot obtain the stature measurement for a reason other than EC.

- $\quad$ NS $=$ Not Straight: Select NS to code an inaccurate stature measurement if the SP's posture was not straight.

The drop-down list changes depending on whether or not a value is entered. Specifically, if the height and/or length value is zero, the drop down will provide the options CNO or EC (Exhibit 4-8a). When a non-zero value is entered in the height and/or length, the drop-down list will consist of only NS (Exhibit 4-8b).

Exhibit 4-8a. Stature screen - Recumbent length blank comments

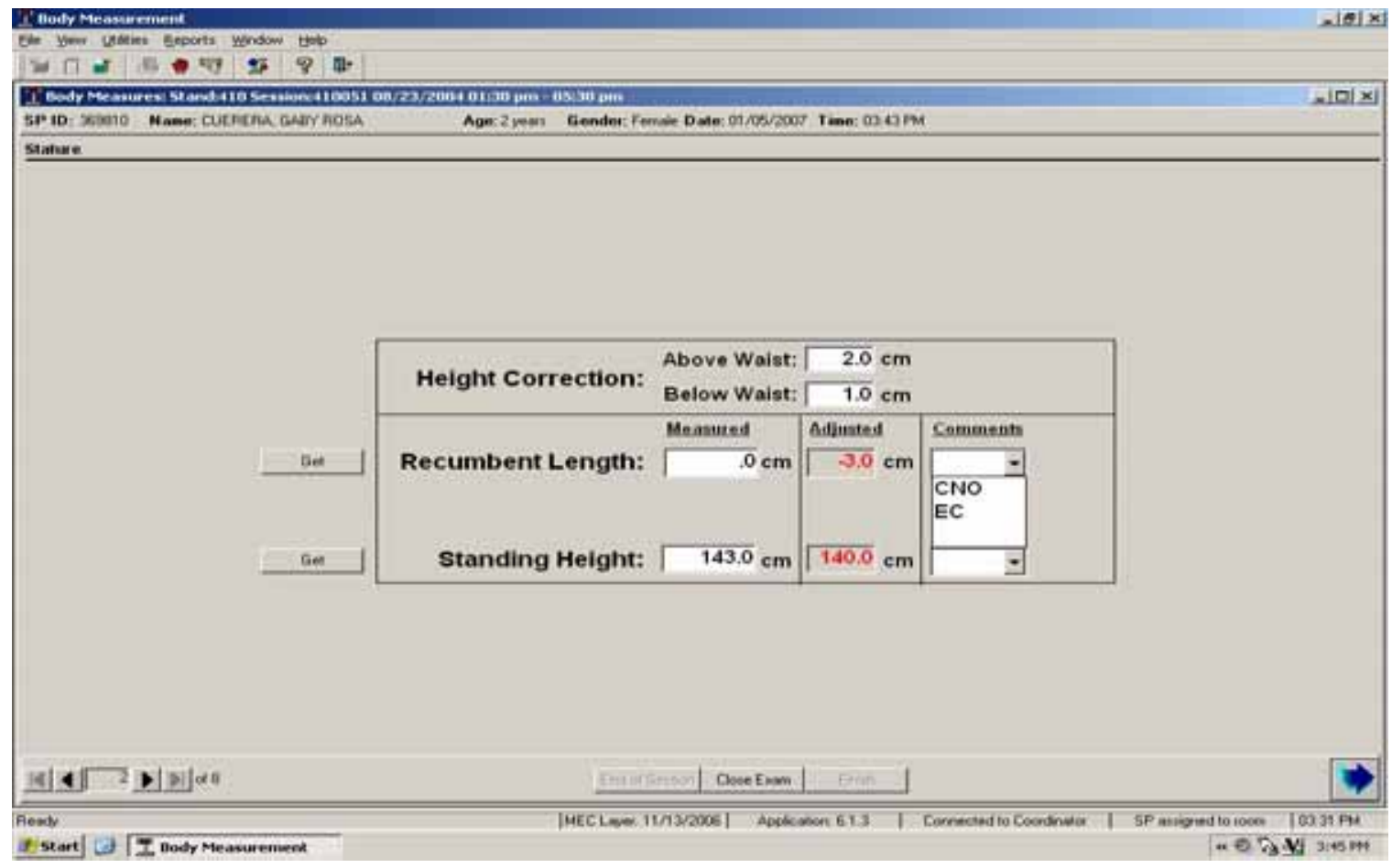


Exhibit 4-8b. Stature screen - Standing height value comments

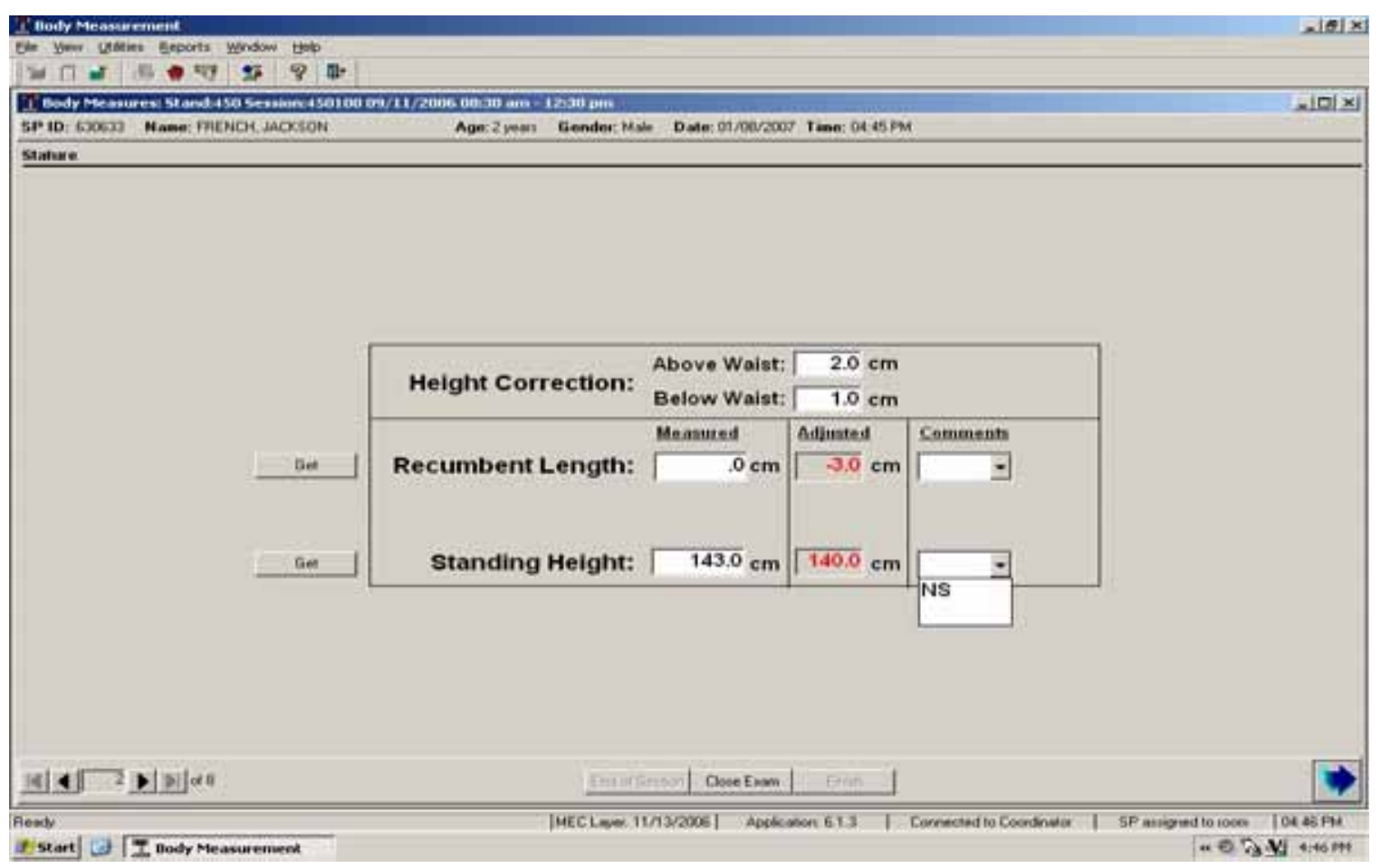

Following the Stature screen, the recorder will manually enter the remaining anthropometry measurements into ISIS. 
The next screen is entitled, Sitting Measures (Exhibit 4-9). This screen displays the Upper Leg Length measure, which is collected on SPs aged 8 years and older. After the recorder enters the upper leg length, ISIS will repeat the number aloud for confirmation.

If you cannot obtain the measure for some reason, e.g., SP refusal or physical limitation, select CNO (Could Not Obtain) from the Comment box located beside the measurement field.

Exhibit 4-9. Sitting measures screen

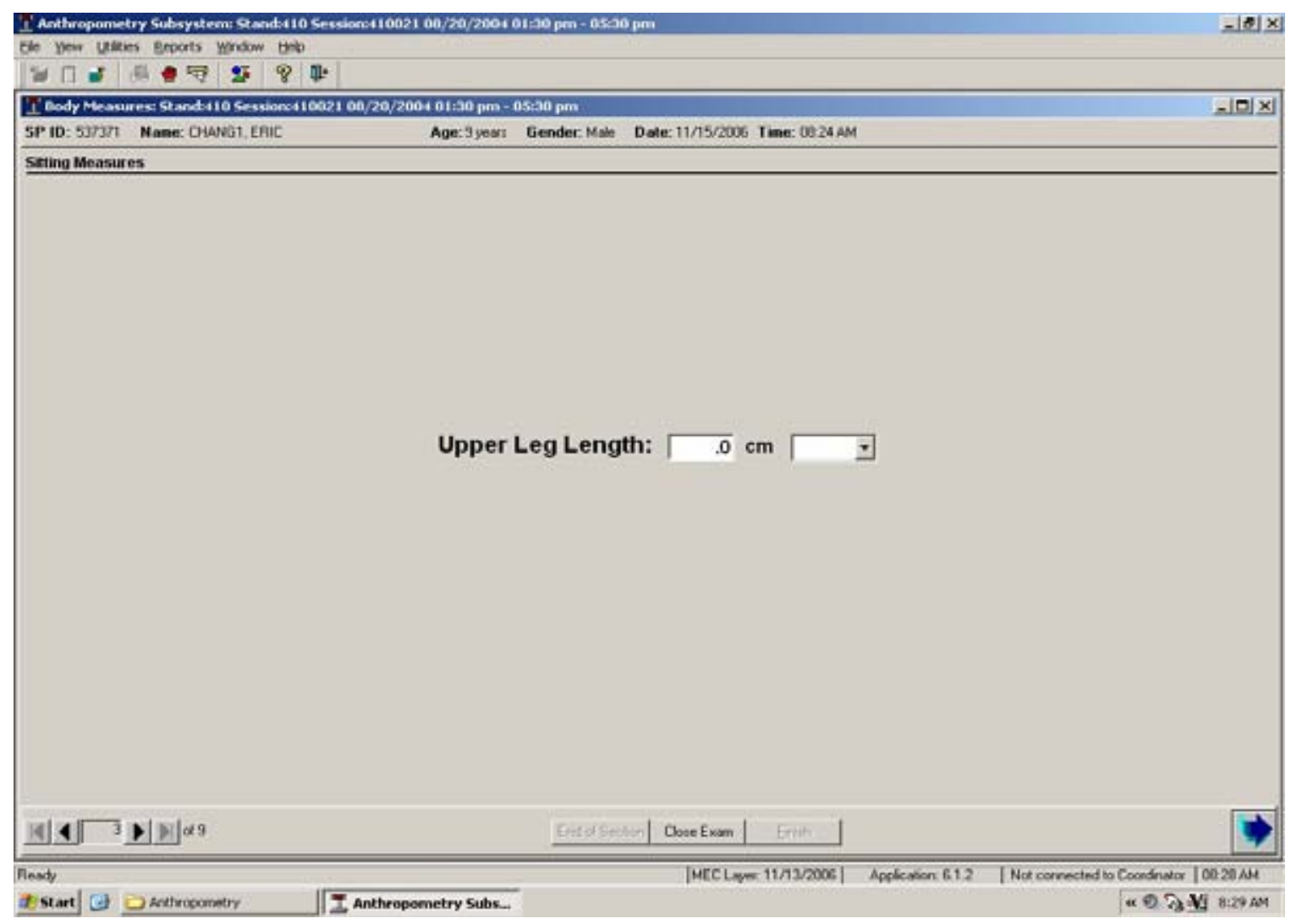




\subsection{Upper Arm Length Screen}

Following Upper Leg Length is the Upper Arm Length screen (Exhibit 4-10). This screen is displayed for all SPs aged 2 months and older. After the recorder enters the upper arm length, ISIS will call out both the number entered and the midpoint of this measurement.

If you cannot obtain the measure for some reason, e.g., SP refusal or physical limitation, select CNO (Could Not Obtain) from the corresponding Comment box.

Exhibit 4-10. Upper arm length screen

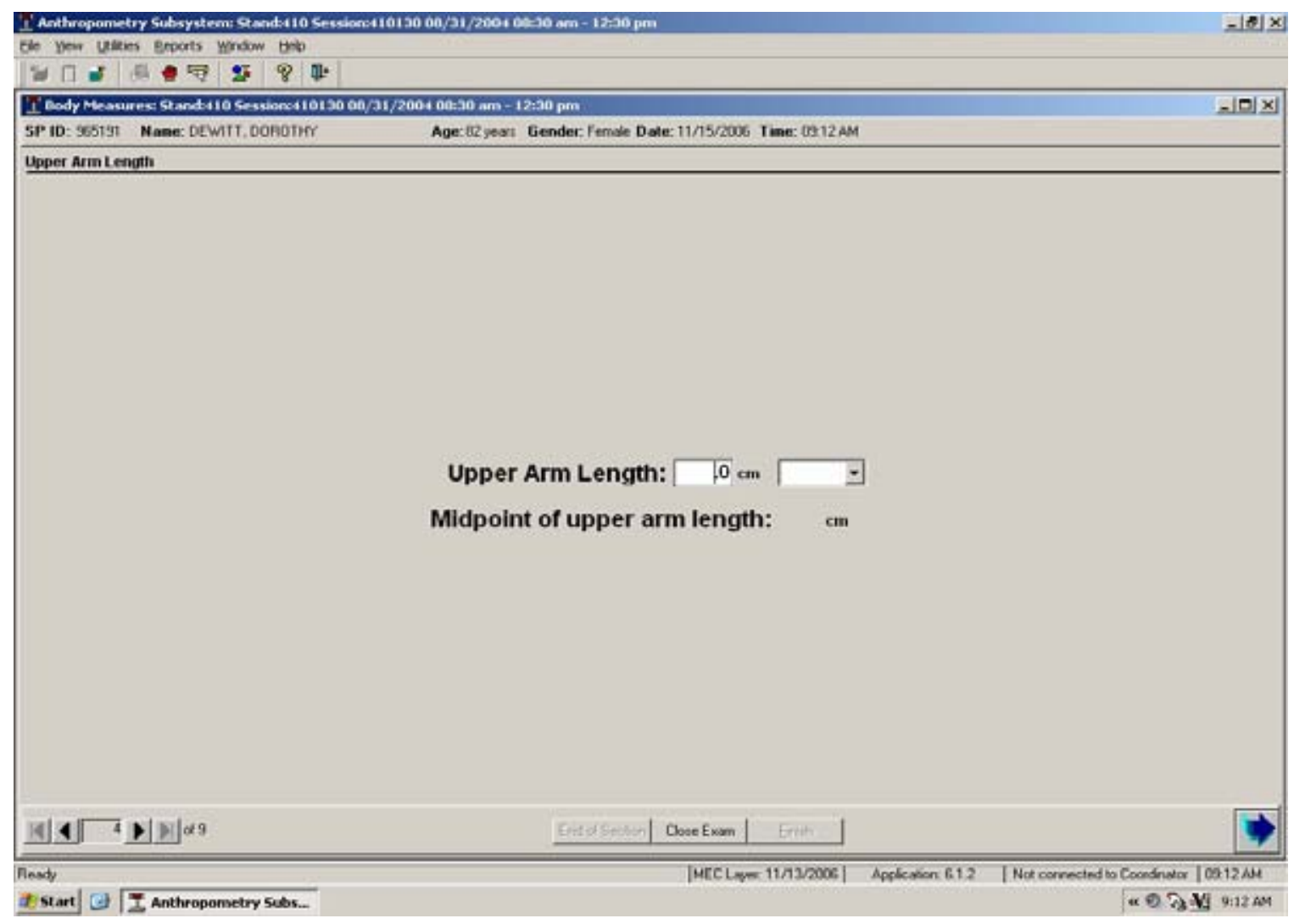




\section{Circumference Measures Screen}

The Circumference Measures screen (Exhibit 4-11) is also displayed for SPs aged 2 months and older. This screen contains two measures: Arm Circumference and Waist Circumference. The current anthropometry protocol requires the arm circumference measure for SPs beginning at 2 months of age and the waist circumference measure for SPs beginning at 2 years of age. After the recorder enters each measurement, ISIS will repeat the number aloud for confirmation.

If you cannot obtain the measure for some reason, e.g., SP refusal or physical limitation, select CNO (Could Not Obtain) from the corresponding Comment box.

To move the screen cursor from one measurement field to the next, press "Enter" or "Tab" on the keyboard or click the cursor on the desired field.

Exhibit 4-11. Circumference measures screen

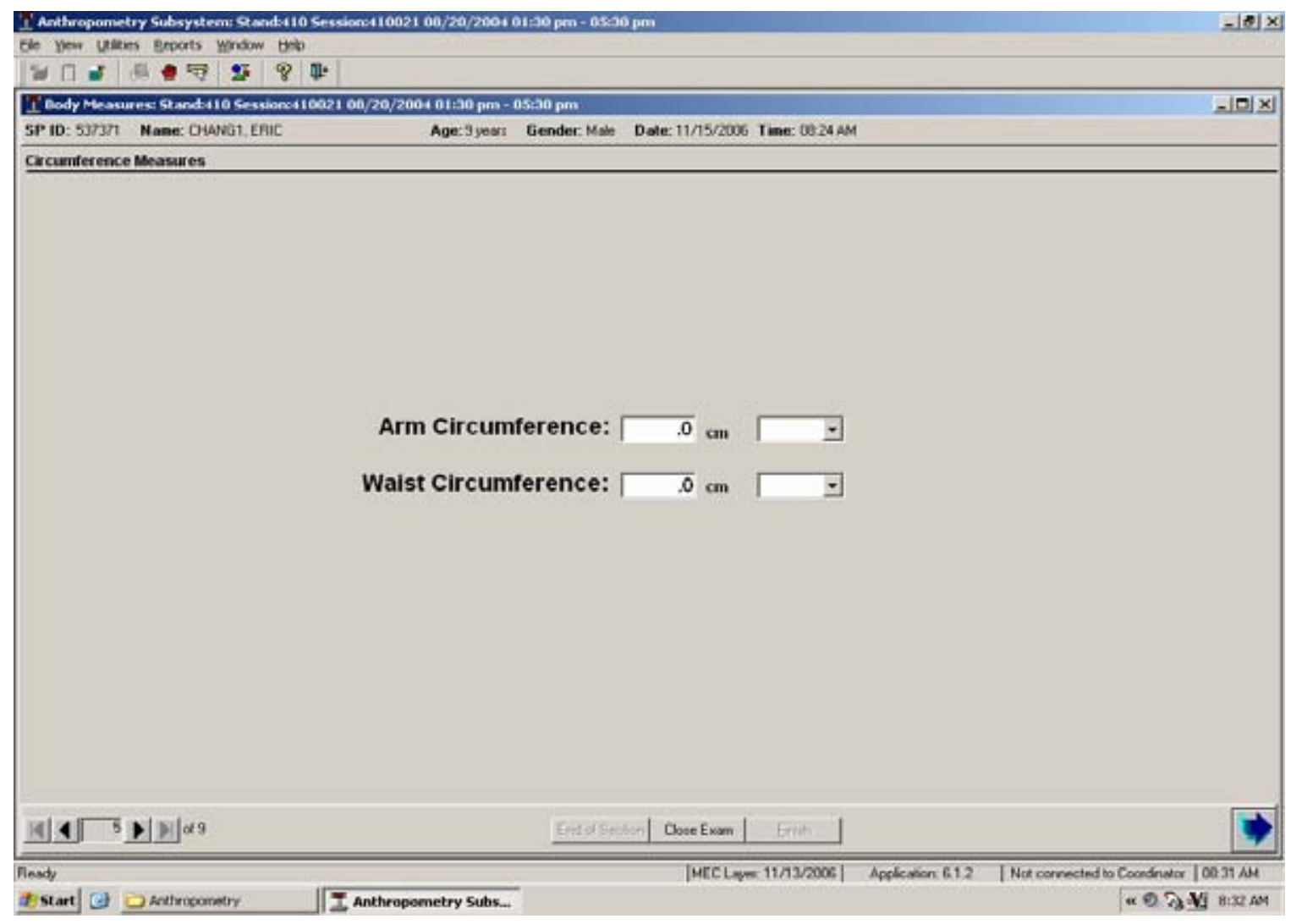


Next, ISIS will display the Skinfold Measures screen (Exhibit 4-12) for SPs aged 2 months and older. This screen contains two skinfold measures: Triceps Skinfold and Subscapular Skinfold. ISIS will continue to repeat each measurement aloud after the recorder enters the number on the screen.

If you cannot obtain the measure for some reason, e.g., SP refusal or physical limitation, select CNO (Could Not Obtain) from the corresponding Comment box.

If the skinfold measurement exceeds $45.0 \mathrm{~mm}$, select EC (Exceeds Capacity) from the Comment box. Using the existing skinfold calipers, measurements greater than $45.0 \mathrm{~mm}$ will not be considered accurate. ISIS has been programmed accordingly to only accept entries less than or equal to 45.0 .

To move the screen cursor from one measurement field to the next, press "Enter" or "Tab" on the keyboard or click the cursor on the desired field.

Exhibit 4-12. Skinfold measures screen

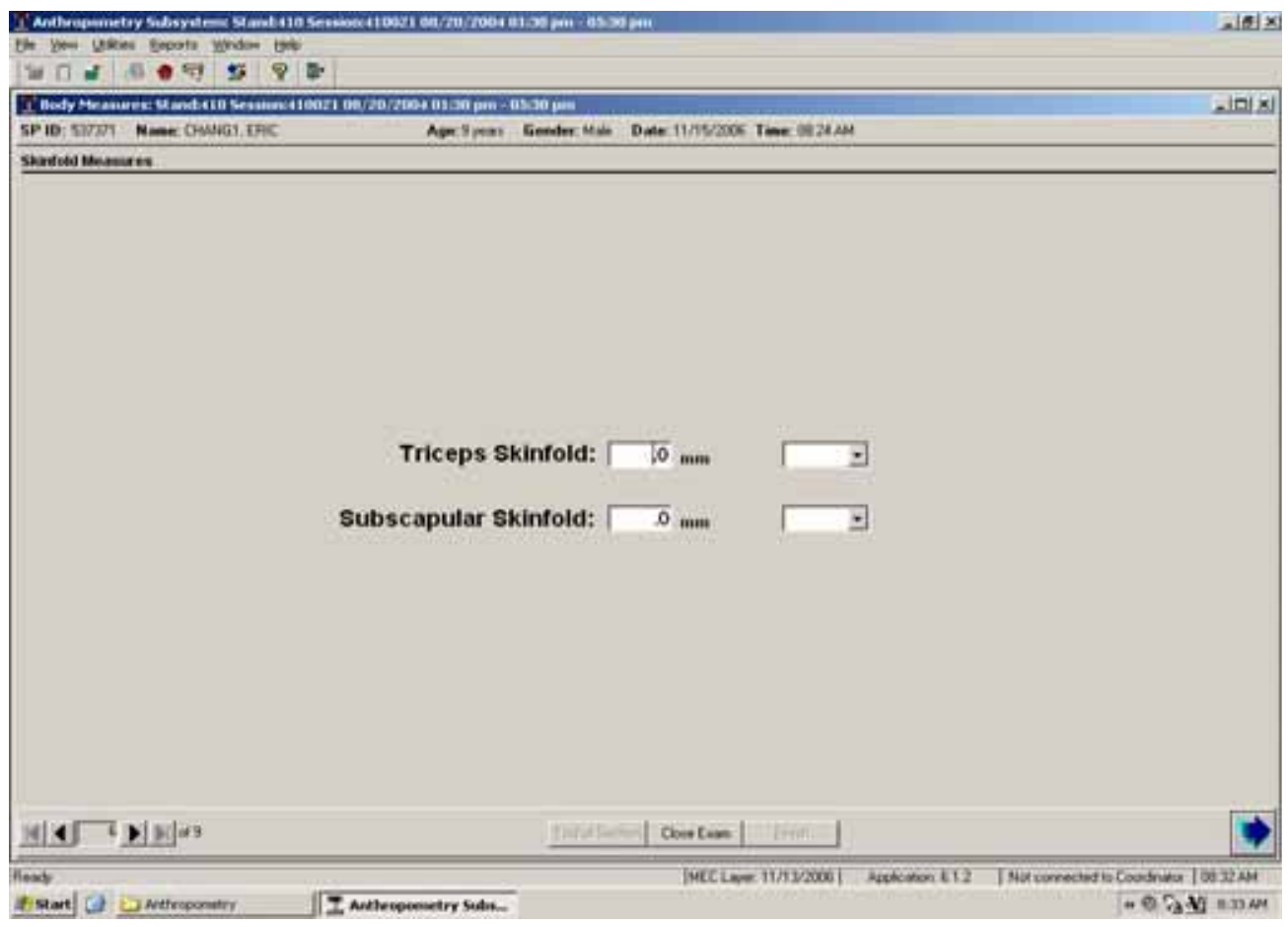


Following the measurement screens, ISIS will present the SP Information (Exhibit 4-13) screen which offers the question, "Do you want to know your height and weight?" The recorder will read the question to the SP and click the "Yes" or "No" radio button. This will trigger the following responses:

- YES - ISIS will show two boxes: Weight in both pounds and kilograms; and Height in both English and metric equivalents.

- NO - ISIS will be ready to advance to the next screen.

Exhibit 4-13. SP information screen

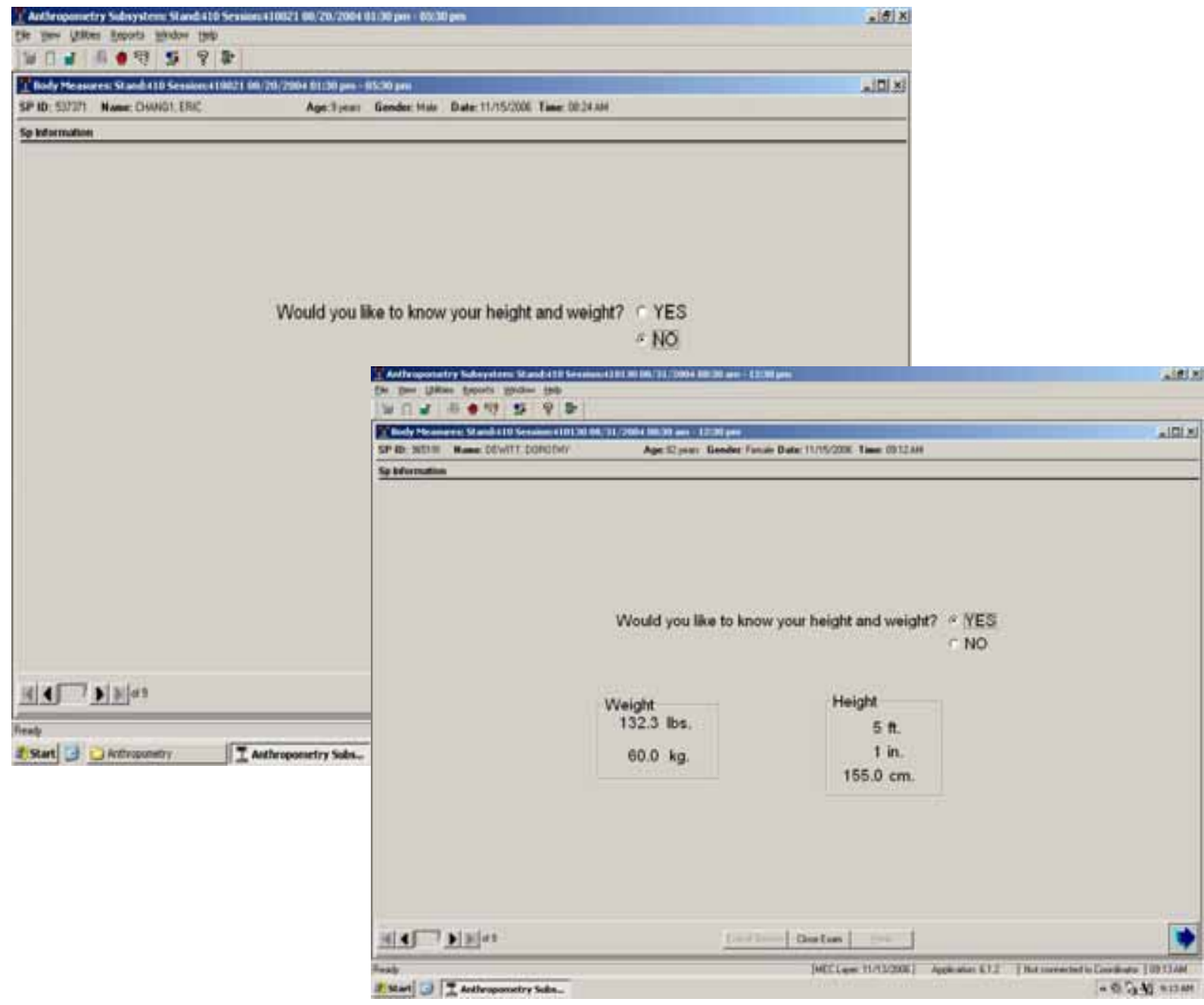


The Amputations screen (Exhibit 4-14) is an observation screen for the exam staff to complete without asking the SP directly. The recorder will mark the appropriate Yes or No radio button beside "Amputations?" This will trigger the following responses:

- Amputations: YES - ISIS will add four questions to the screen, which the recorder must answer by clicking "Yes," "No," or "Could Not Obtain" for each:

- $\quad$ Upper Right Extremity?

- $\quad$ Upper Left Extremity?

- $\quad$ Lower Right Extremity?

- $\quad$ Lower Left Extremity?

- Upper Extremities: YES - Clicking "Yes" to either of the upper extremities will prompt ISIS to add two more questions: Above Elbow? Below Elbow? The recorder must mark the Yes or No radio buttons beside each.

- Lower Extremities: YES - Clicking "Yes" to either of the lower extremities will prompt ISIS to add two more questions: Above Knee? Below Knee? The recorder must mark the Yes or No radio buttons beside each.

- Amputations: NO - ISIS will be ready to advance to the next screen. 
Exhibit 4-14. Amputations screen

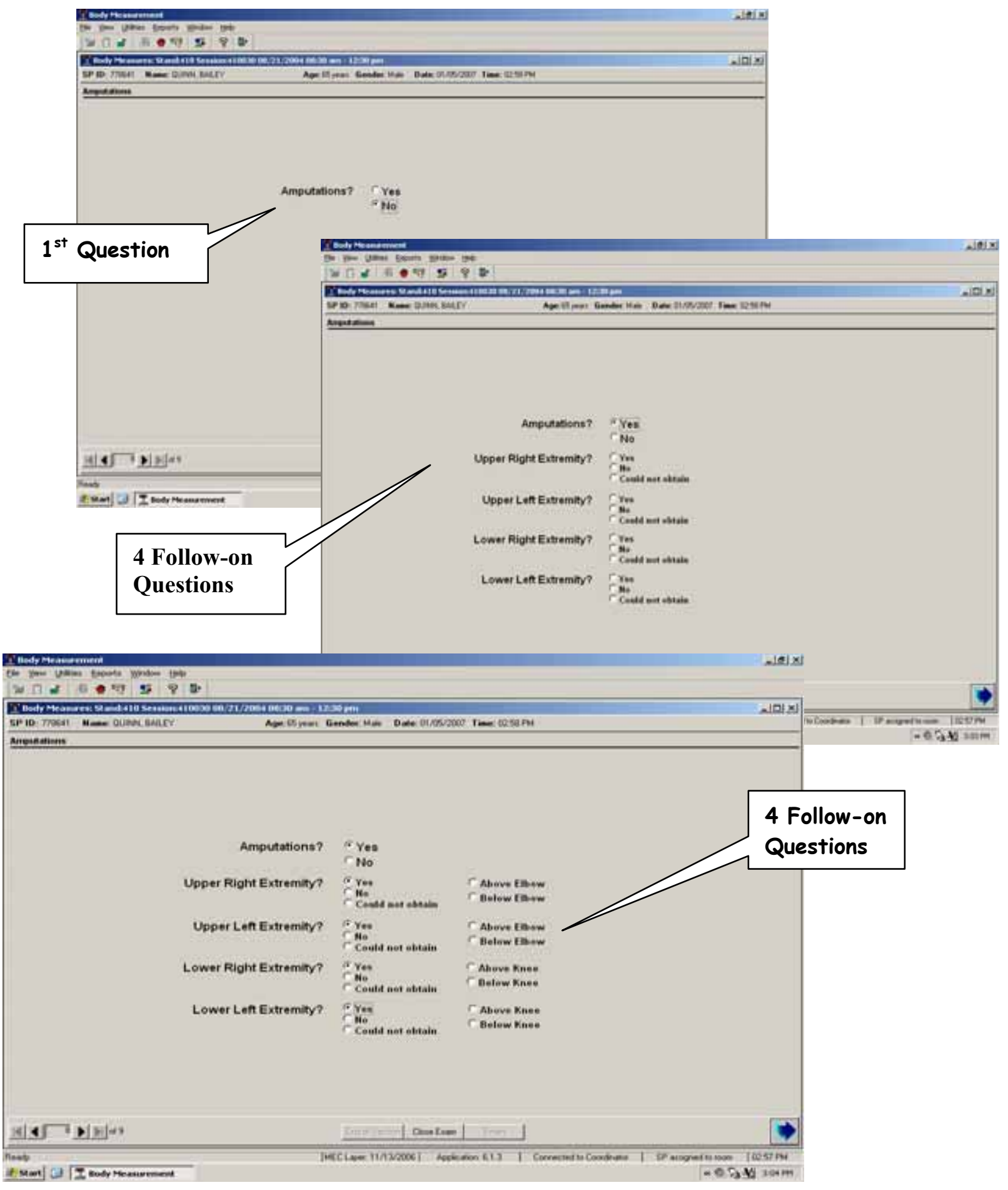


The Anthropometry Component Status (Exhibits 4-15 and 4-16) marks the final ISIS screens. The purpose of these screens is to document the overall status of the anthropometry examination: Complete, Partial, and Not Done. As with all other MEC exam components, ISIS will automatically default to one of these codes.

- Complete: All required measures were captured in ISIS.

- Partial: One or more required measures were NOT captured in ISIS.

- Not Done: No required measures were captured in ISIS.

For Partial and Not Done exams, use the drop-down menu beside the Comments box to select from nine reasons: safety exclusion, SP refusal, no time, physical limitation, communication problem, equipment failure, SP ill/emergency, interrupted, or Other, specify. If you choose the "Other, specify" comment you must enter a description. Be as brief as possible. Only select "Other, specify" if the comment does not fit into one of the defined comments.

The following are two common scenarios regarding reasons for Partial status exams:

- Partial status example \#1: If CNO or EC was entered for one of the measures because it was physically impossible for the examiner to obtain the measurement, then ISIS will code the exam status as Partial and the recorder should code the reason as Physical Limitation.

- Partial status example \#2: If CNO was entered for one of the measures because the SP refused to allow the examiner to obtain the measurement, then ISIS will code the exam status as Partial and the recorder should code the reason as SP Refusal.

Lastly, click "Finish" to end the examination. Wait for a message from the coordinator indicating where to direct the SP. Thank the SP for participating and escort him or her to the next exam room or to the coordinator. 
Exhibit 4-15. Anthropometry component status screen - complete

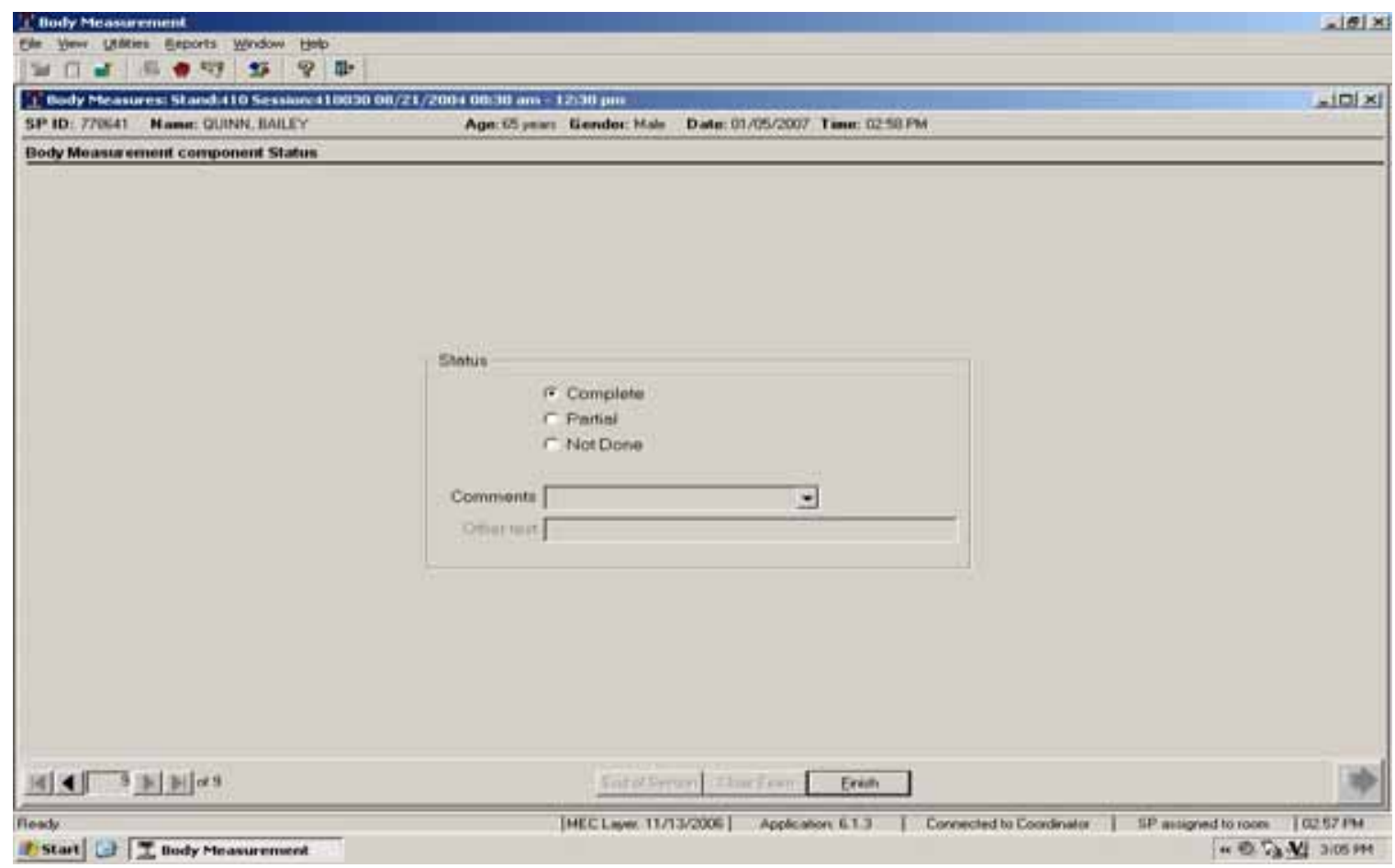

Exhibit 4-16. Anthropometry component status screen - partial

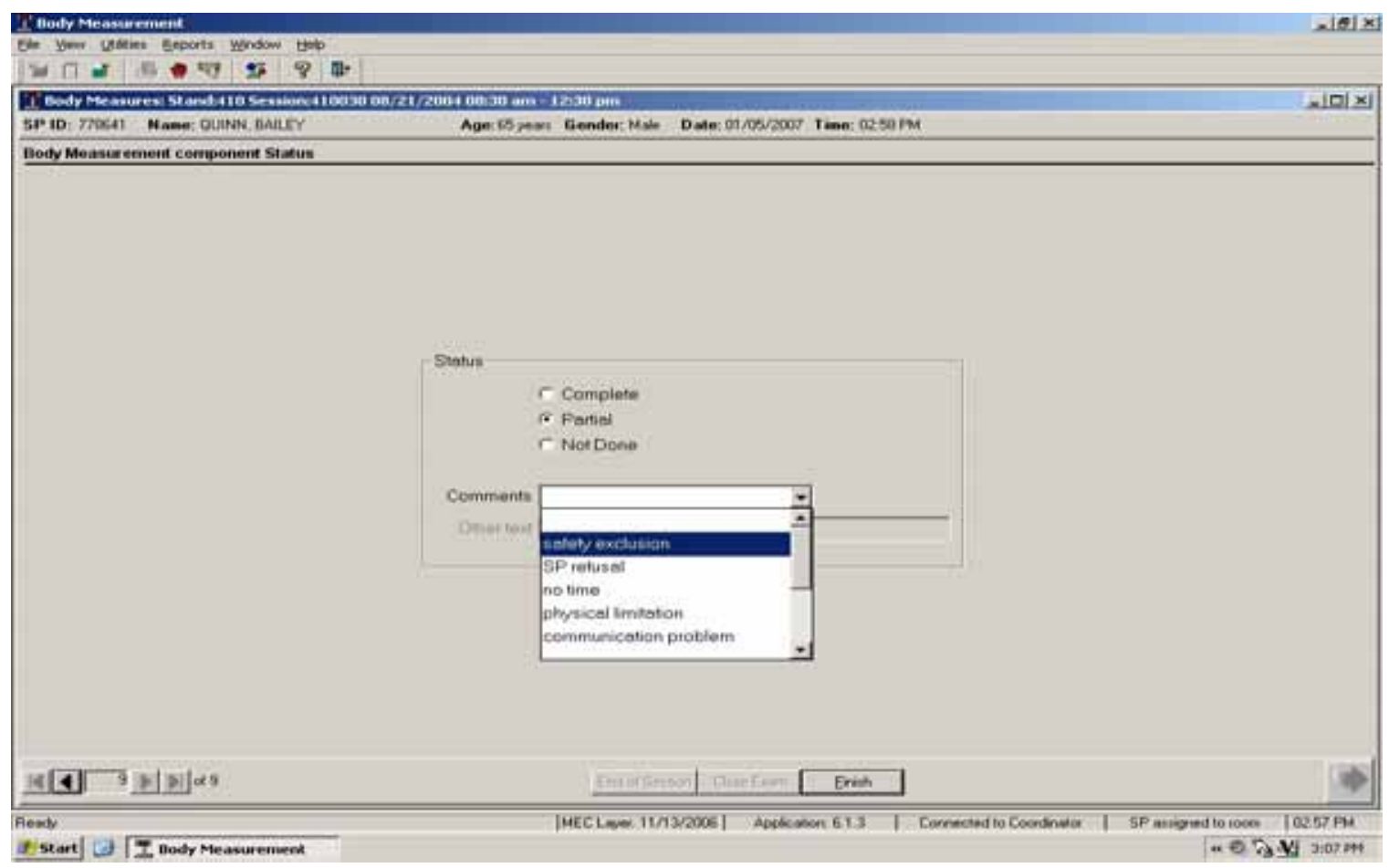




\section{QUALITY CONTROL}

\subsection{Introduction to Quality Control for Anthropometry}

Quality control procedures ensure the collection and documentation of accurate, reliable data. The NHANES anthropometry data are among the most widely used data collected in the study. Your role as an examiner or recorder is crucial to the success of this component. The anthropometry protocol requires the examiner and recorder to work together as a team (See Chapter 3, Examination Protocol.).

In anthropometrics, the most common errors involve body positioning, locating and marking body landmarks, reading measurement results, and recording examination results in ISIS. The methods used in NHANES were developed to minimize inter-examiner variability, reduce error, and ensure data quality. In addition to the standardized examination protocol, the anthropometry component incorporates specific quality control procedures.

\subsection{Equipment Calibrations}

Routine calibrations of the anthropometry equipment ensure that the equipment produces accurate measures. The following pieces of equipment require calibration at specific points during a stand: digital weight scale, portable scales, stadiometer, infantometer, and skinfold calipers. Table 5-1 summarizes the calibration schedule and requirements for these equipment pieces.

Sections 5.2.1-5.2.6 detail the required calibration procedures for the equipment listed in Table 5-1. The technologist will record the results of all calibrations on the ISIS screen entitled, "Body Measures Quality Control Checks." To access this screen, select the Quality Control option from the main Utilities menu. The Body Measures Quality Control Checks dialog box (Exhibit 5-1) contains individual tabs designated for the Start of Stand, Daily, Weekly, Middle of Stand, End of Stand, and Equipment Swap calibration procedures. 
Table 5-1. Anthropometry equipment calibrations

\begin{tabular}{|c|c|c|}
\hline $\begin{array}{c}\text { Calibration } \\
\text { Schedule }\end{array}$ & Equipment & Calibration Requirements \\
\hline Start of Stand & $\begin{array}{l}\text { Digital weight scale } \\
\text { Portable scales } \\
\text { Stadiometer } \\
\text { Infantometer } \\
\text { Skinfold calipers }\end{array}$ & $\begin{array}{l}15,10 \mathrm{~kg} \text { weights, acceptable range: } 149.85-150.15 \mathrm{~kg} \\
11,10 \mathrm{~kg} \text { weights, acceptable range: } 109.50-110.50 \mathrm{~kg} \\
80 \mathrm{~cm} \text { rod } \\
\text { Foot piece to headboard: MEC } 1: 16 \mathrm{~cm} \text {; MEC 2, MEC } 3: 0 \mathrm{~cm} \\
\text { Step 1: } 9.8-10.5 ; \\
\text { Step 2: } 19.8-20.5 ; \\
\text { Step 3: } 29.9-30.5 ; \\
\text { Step 4: } 39.8-40.4\end{array}$ \\
\hline Daily & Digital weight scale & $5,10 \mathrm{~kg}$ weights, acceptable range: $49.70-50.30 \mathrm{~kg}$ \\
\hline Weekly & $\begin{array}{l}\text { Stadiometer } \\
\text { Infantometer } \\
\text { Skinfold calipers }\end{array}$ & $\begin{array}{l}80 \mathrm{~cm} \text { rod } \\
\text { Foot piece to headboard: MEC 1: } 16 \mathrm{~cm} \text {; MEC 2, MEC 3: } 0 \mathrm{~cm} \\
\text { Step 1: } 9.8-10.5 \text {; } \\
\text { Step 2: } 19.8-20.5 \text {; } \\
\text { Step 3: } 29.9-30.5 \text {; } \\
\text { Step 4: } 39.8-40.4\end{array}$ \\
\hline Mid-Stand & Digital weight scale & $15,10 \mathrm{~kg}$ weights, acceptable range: $149.85-150.15 \mathrm{~kg}$ \\
\hline End of Stand & Digital weight scale & $15,10 \mathrm{~kg}$ weights, acceptable range: $149.85-150.15 \mathrm{~kg}$ \\
\hline
\end{tabular}

Exhibit 5-1. Body measures quality control checks box

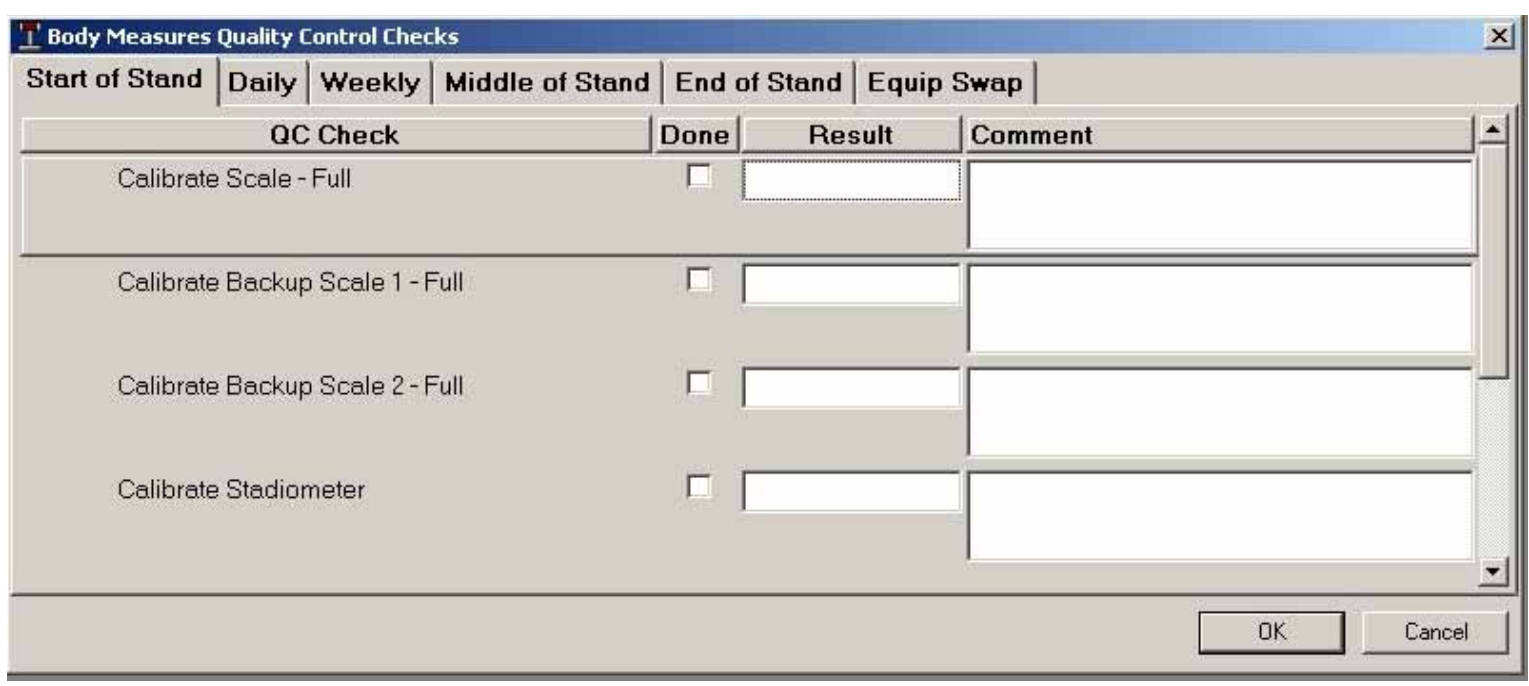


To exit the Body Measures Quality Control Checks dialog box, click "OK." If any required procedures are not checked as "Done," ISIS will present the following pop-up reminder window:

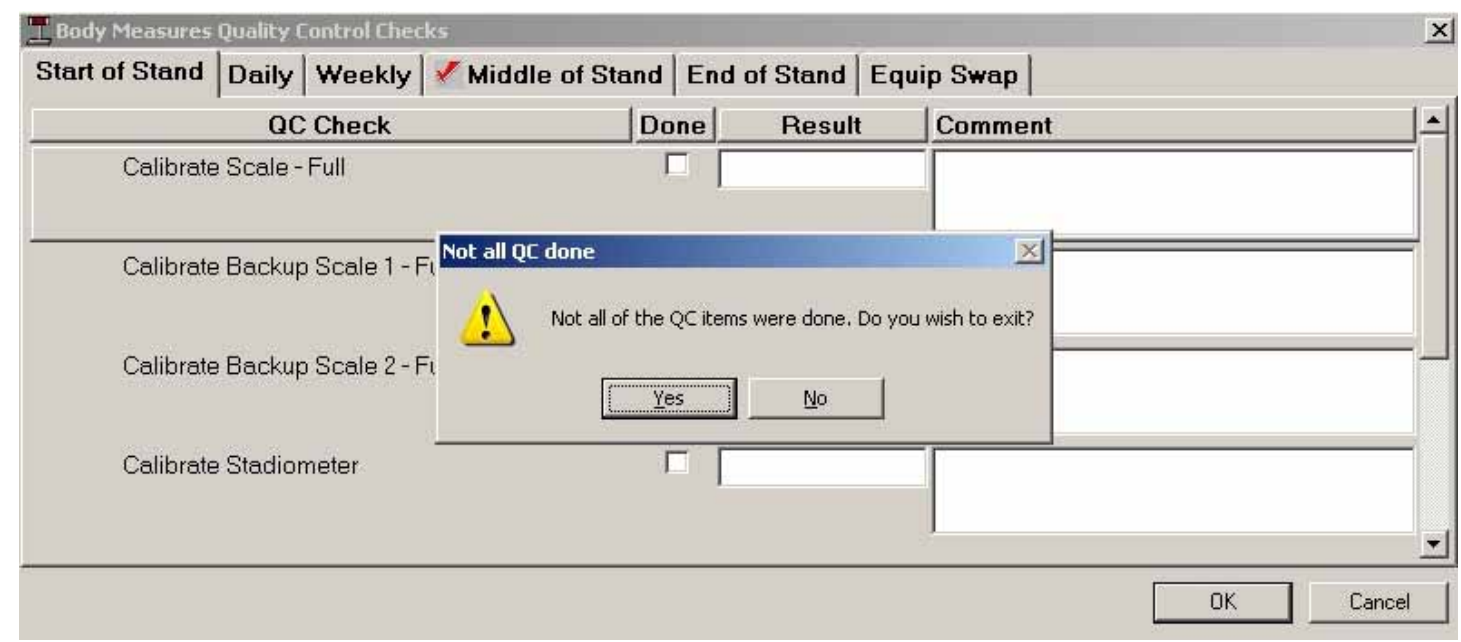

To return to the QC dialog box, click "Cancel," perform the required procedure(s), and check the corresponding "Done" box. If it is time to perform Middle of Stand QC, when you attempt to exit the QC dialog box ISIS will present a similar pop-up window that includes a reminder to perform the mid-stand QC procedure, "Calibrate Scale - Full."

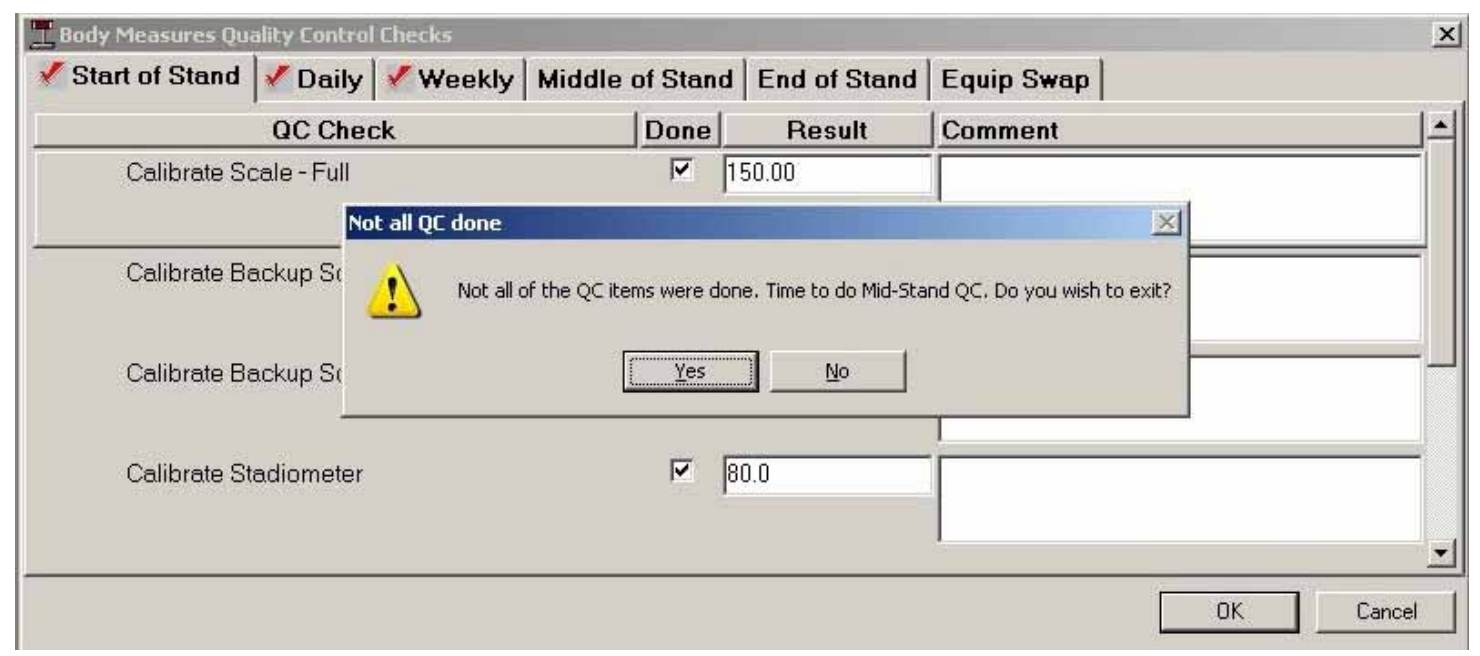

Finally, if you need to exit the Body Measures QC utility without completing all of the required procedures listed in the QC dialog box: click OK to close the dialog box and click OK to close the pop-up reminder window. ISIS will then return you to the main component screen. 


\subsection{1}

\section{Digital Weight Scale}

Full calibration of the digital weight scale is required at the start, middle, and end of a stand. In addition, a rough calibration must be done daily prior to the first exam session.

\section{Full Calibration: Start, Middle, End of Stand}

- Carefully place all 15 of the 10 kilogram calibration weights on the scale. To ensure an accurate measurement reading, the weights must be placed evenly distributed over the center area of the scale.

- Click on the appropriate tab (i.e., "Start of Stand," "Middle of Stand," or "End of Stand)" in the ISIS QC Checks dialog box (Exhibit 5-2).

- Click the "Done" box beside "Calibrate Scale - Full" to capture the value directly into ISIS under the Result field.

- The acceptable calibration weight range for 15, 10 kilogram calibration weights on the digital weight scale is $149.85-150.15 \mathrm{~kg}$.

Exhibit 5-2. Digital weight scale full calibration

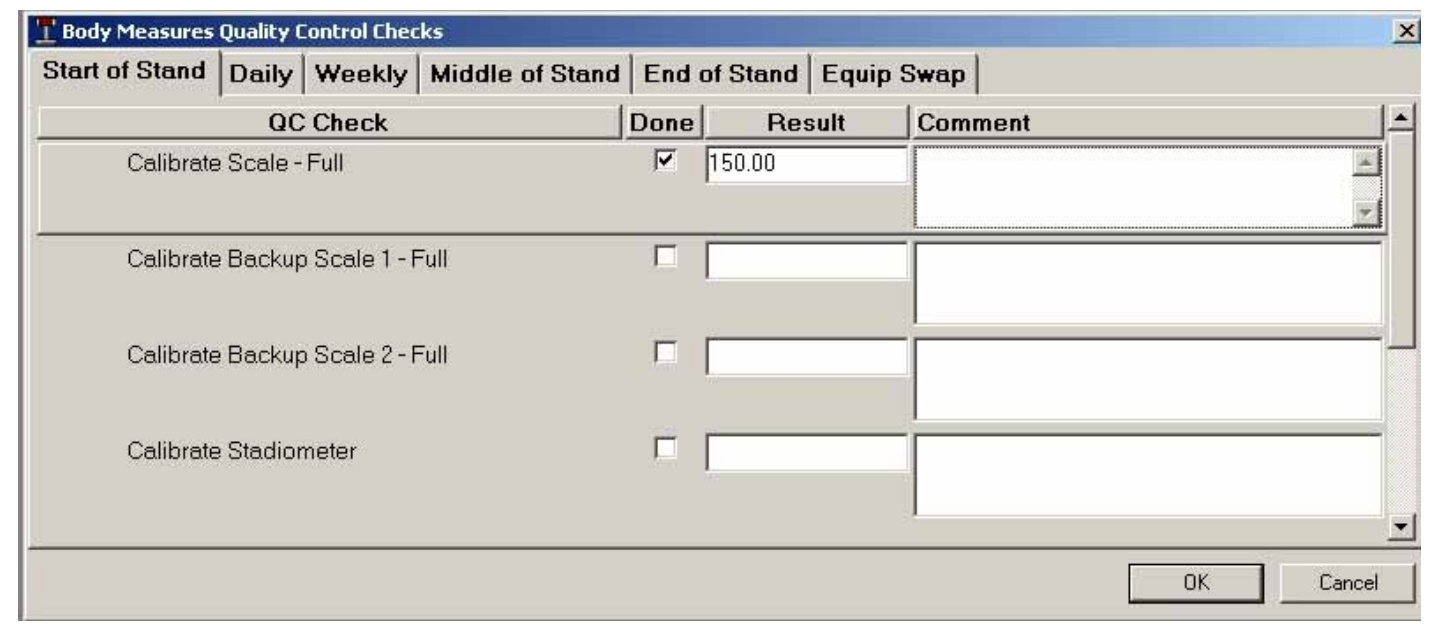

- If the result falls outside the acceptable range, notify the chief technologist, MEC manager, and home office component specialist to have the scale recalibrated by the NCHS engineer or a service representative. Document the issue in the UFO system.

- If the scale is recalibrated at a later date, record the new calibration results under the Equipment Swap tab of the QC Checks dialog box (see Section 5.2.6). 


\section{Rough Calibration: Daily}

- Carefully place 5 of the 10 kilogram calibration weights on the scale. To ensure an accurate measurement reading, the weights must be placed evenly distributed over the center area of the scale.

- Click on the "Daily" tab in the ISIS QC Checks dialog box (Exhibit 5-3).

- Click the "Done" box beside "Calibrate Scale - Daily Weight Check" to capture the value directly into ISIS under the Result field.

- The acceptable calibration weight range for 5, 10 kilogram calibration weights on the digital weight scale is $49.70-50.30 \mathrm{~kg}$.

- If the result falls outside the acceptable range, perform the full calibration procedures described above to determine if the scale remains out of calibration. If the result lies within the acceptable range for the full calibration, repeat the daily calibration procedures with the 5 weights. If the result still lies outside the acceptable range, notify the chief technologist, MEC manager, and home office component specialist to have the scale recalibrated by the NCHS engineer or a service representative. Document the issue in the UFO system.

- If the scale is recalibrated at a later date, record the new calibration results under the Equipment Swap tab of the QC Checks dialog box (see Section 5.2.6).

Exhibit 5-3. Digital weight scale daily calibration

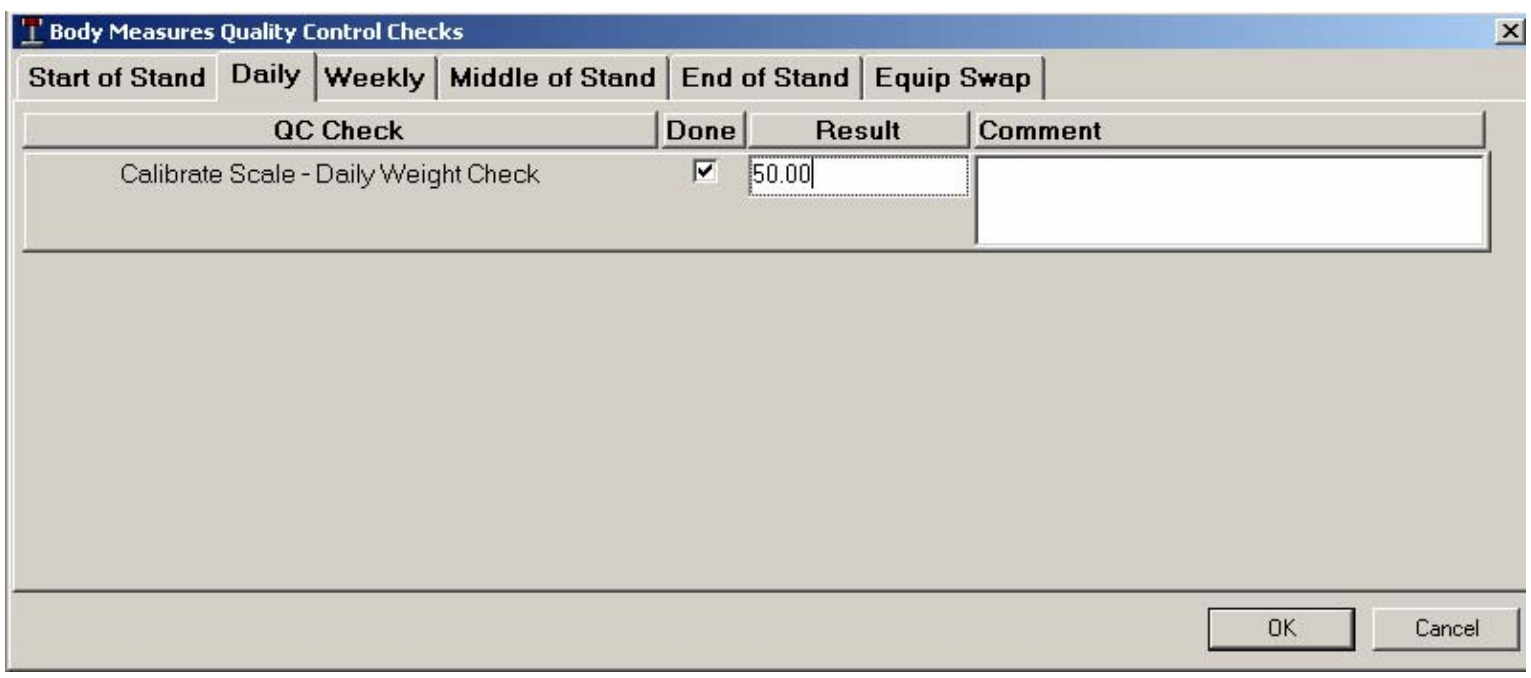




\section{Digital Measurement Device: Pounds (lb) vs. Kilograms (kg)}

The measurement device for the digital weight scale is situated on the wall below the computer monitor (Exhibit 5-4).

Exhibit 5-4. Digital scale measurement device

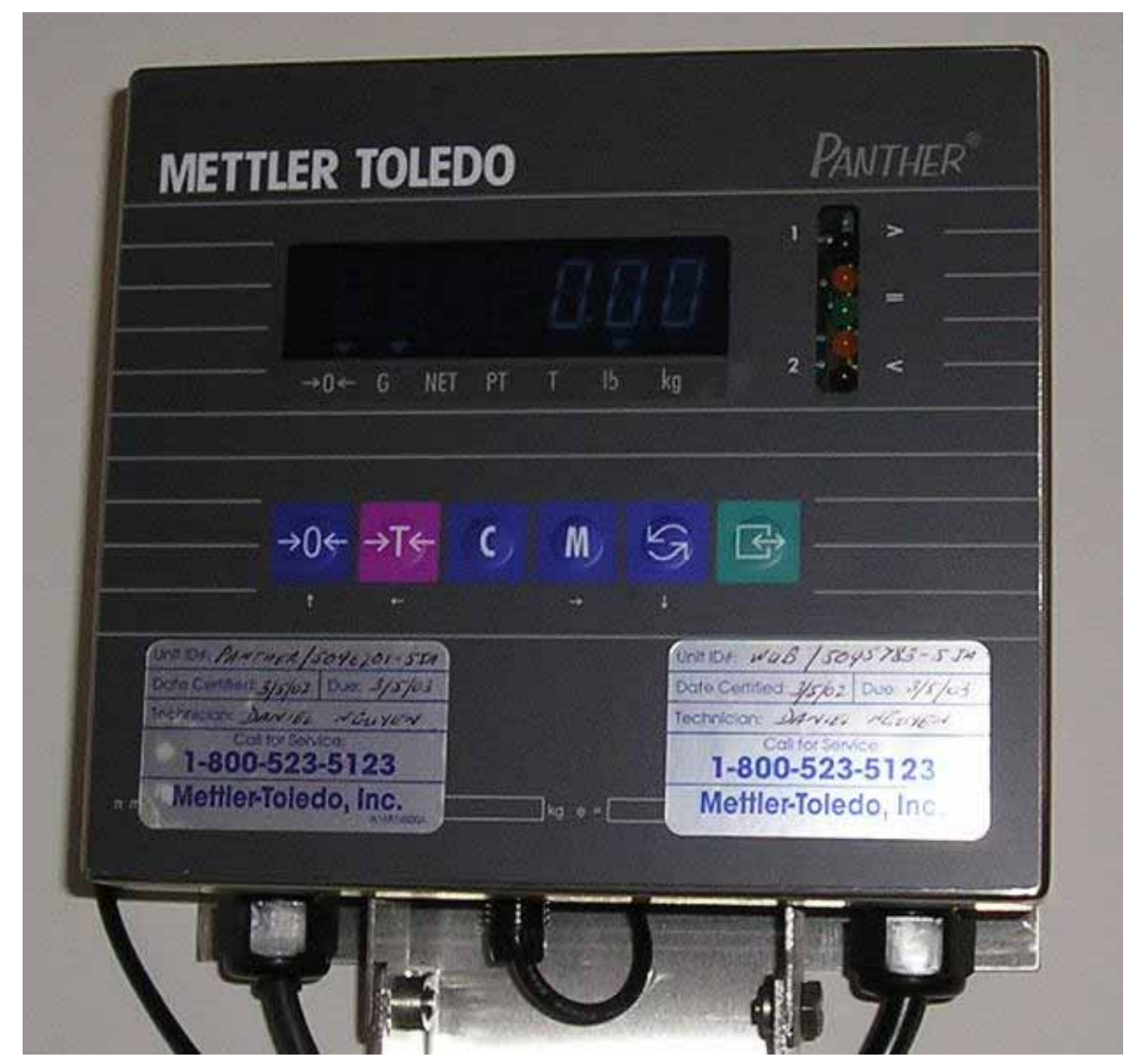

The device contains a row of six buttons. The second button from the right has two arrows that toggle the display from pound to kilogram units. Above this button is an upside down triangle that points to either the "kg" or "lb" display label. Keep the display toggled to kg at all times. 
If the digital measurement device is set to pounds instead of kilograms, when you click "Done" to capture the full or daily scale calibration reading into ISIS the following warning message will appear (Exhibits 5-5 to 5-8).

Exhibit 5-5. Digital weight scale full calibration - scale set to $\mathrm{lb}$

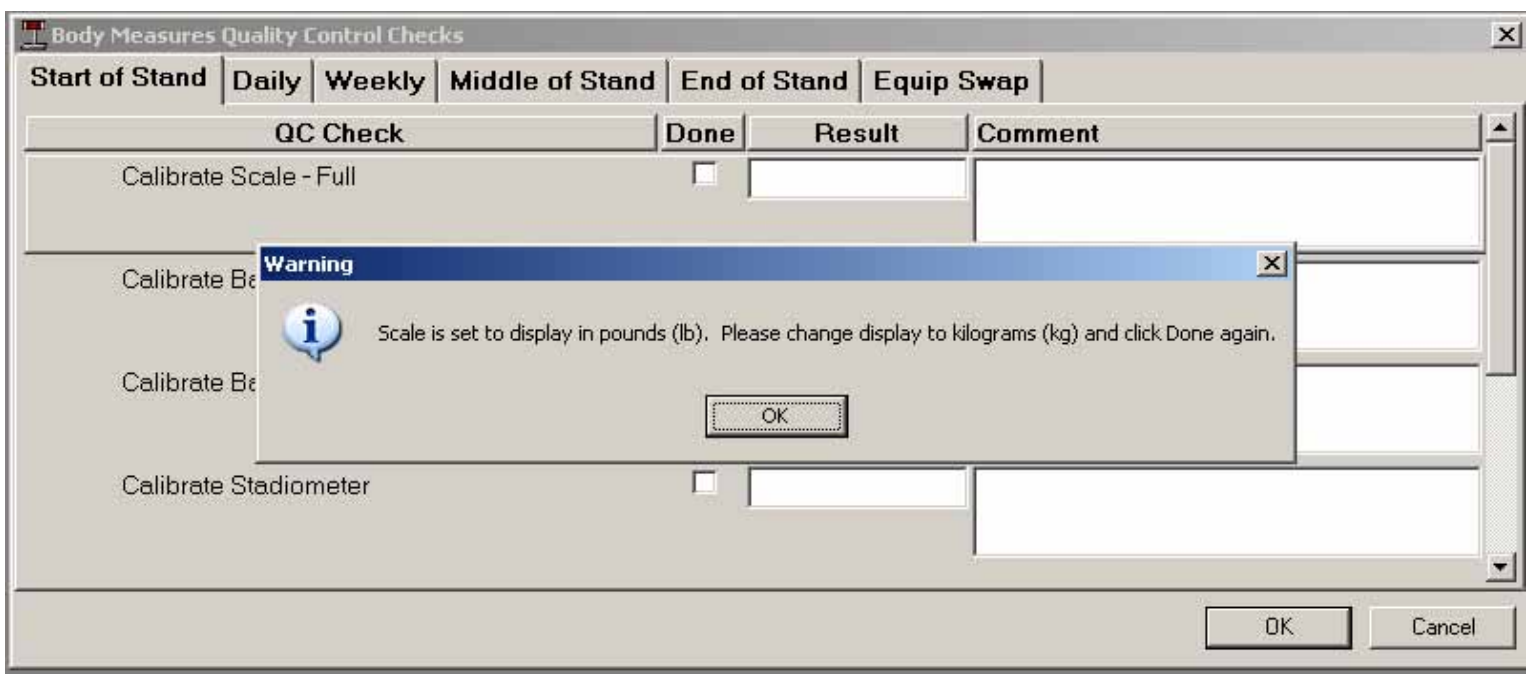

Exhibit 5-6. Digital weight scale daily calibration - scale set to $\mathrm{lb}$

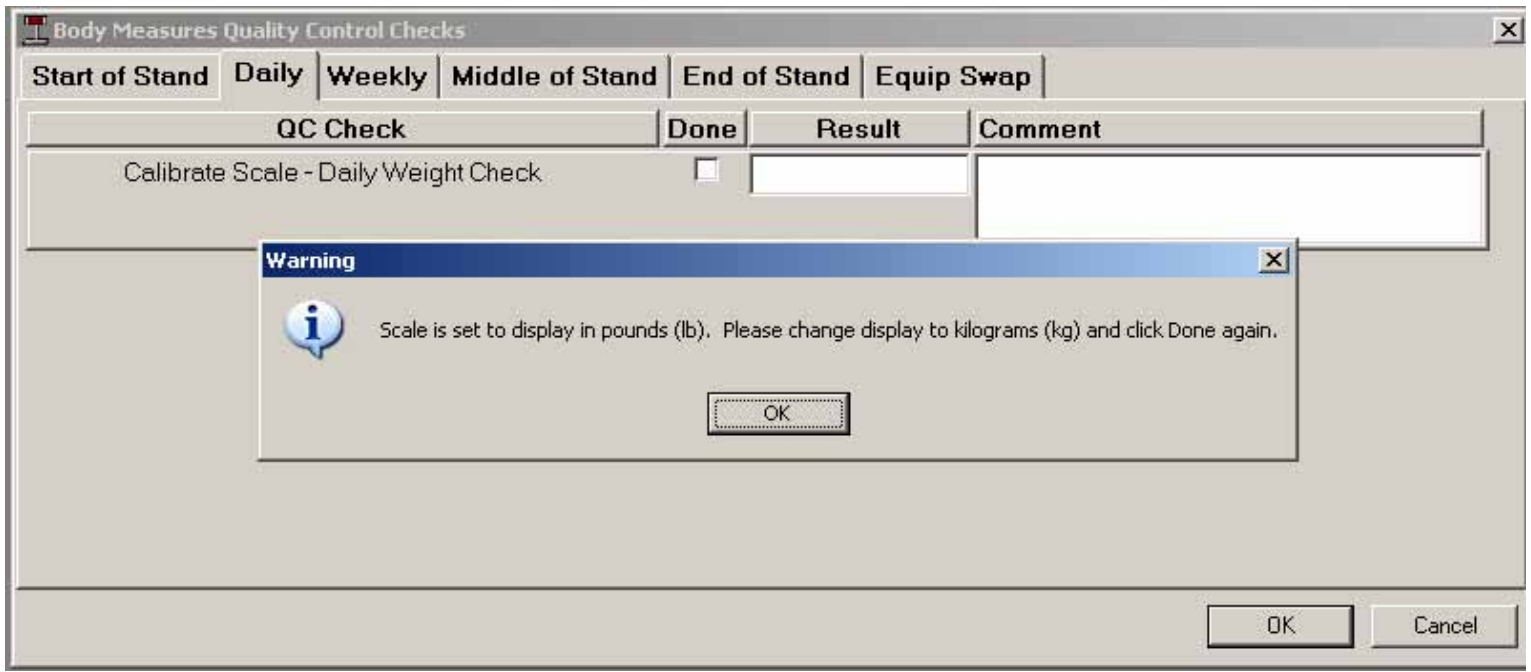


Click OK to this message. Next the following error message will appear:

Exhibit 5-7. Digital weight scale full calibration - lb setting error message

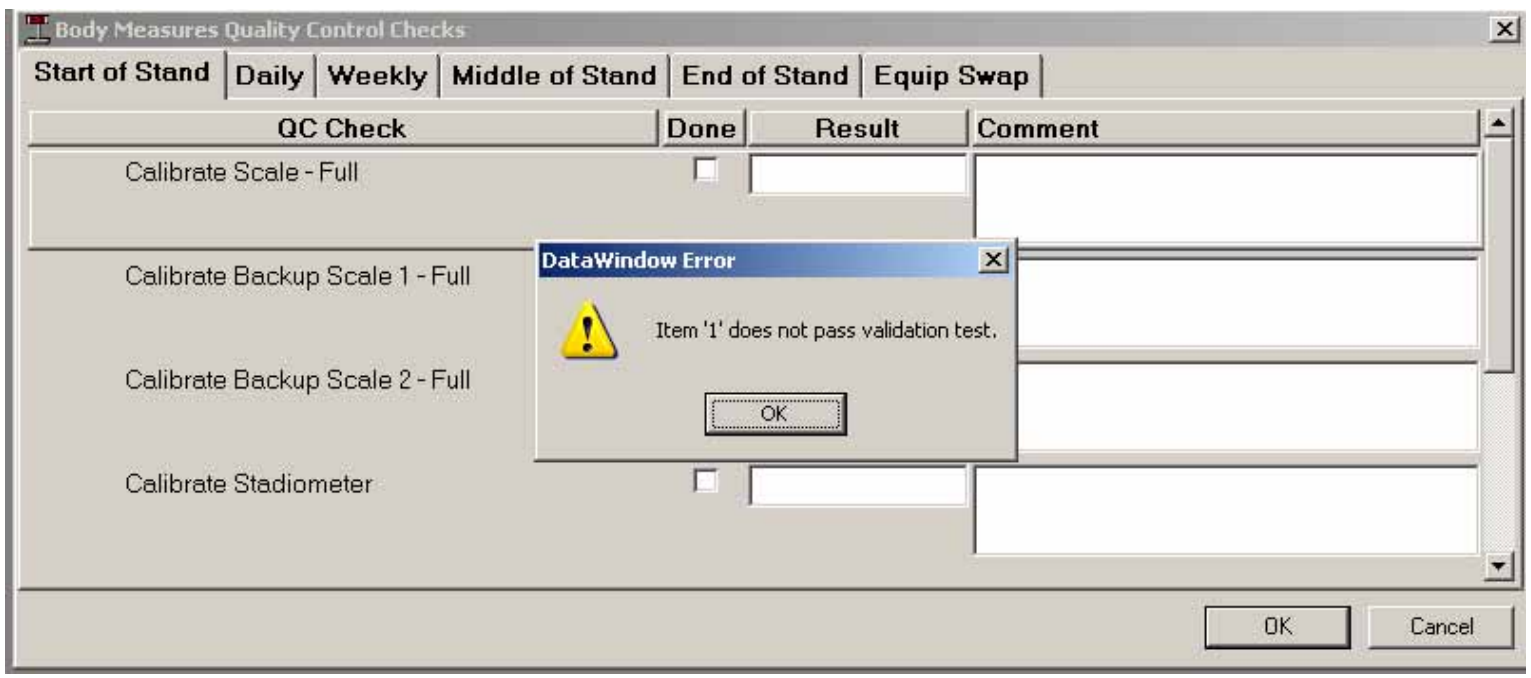

Exhibit 5-8. Digital weight scale daily calibration $-1 \mathrm{~b}$ setting error message

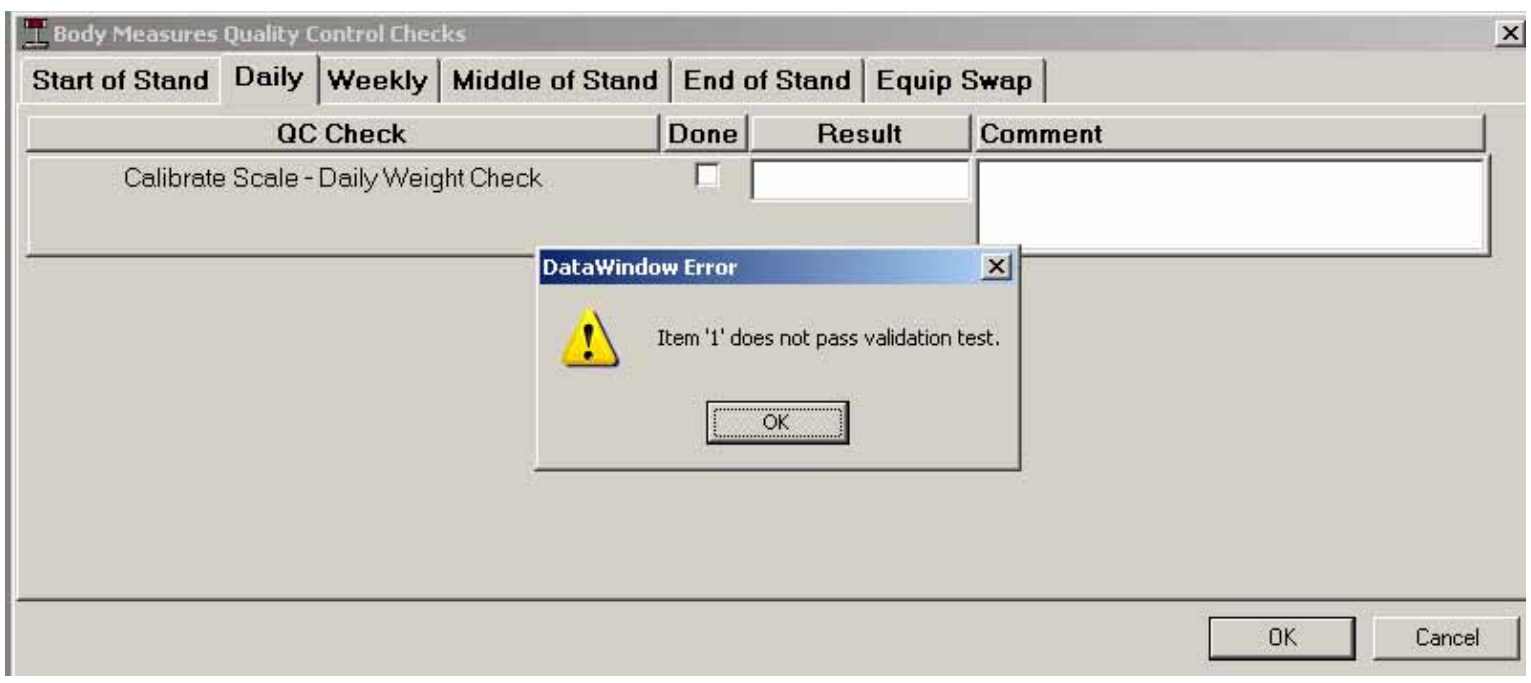

Click OK to this message to uncheck the Done box beside the corresponding "Calibrate Scale - Full" or "Calibrate Scale - Daily Weight Check" QC item.

Change the setting on the digital measurement device to kilograms and retry capturing the $\mathrm{kg}$ value into ISIS. 


\subsubsection{Portable Scales}

\section{Calibration: Start of Stand}

- Place the portable scale on the floor and activate it by lightly touching the surface with your foot.

- Verify that the scale is set to measure weight in kilograms by checking the switch on the back of the scale.

- Wait for the display to read 0.0 .

- Carefully place 11 of the 10 kilogram weights on the scale.

- Click on the "Start of Stand" tab in the ISIS QC Checks dialog box (Exhibit 5-9).

- Click the "Done" box beside "Calibrate Backup Scale 1 - Full."

- When the scale display has stabilized, record the value in the Result field.

- Record the manufacturer serial number in the Comment field.

Exhibit 5-9. Portable scales calibration

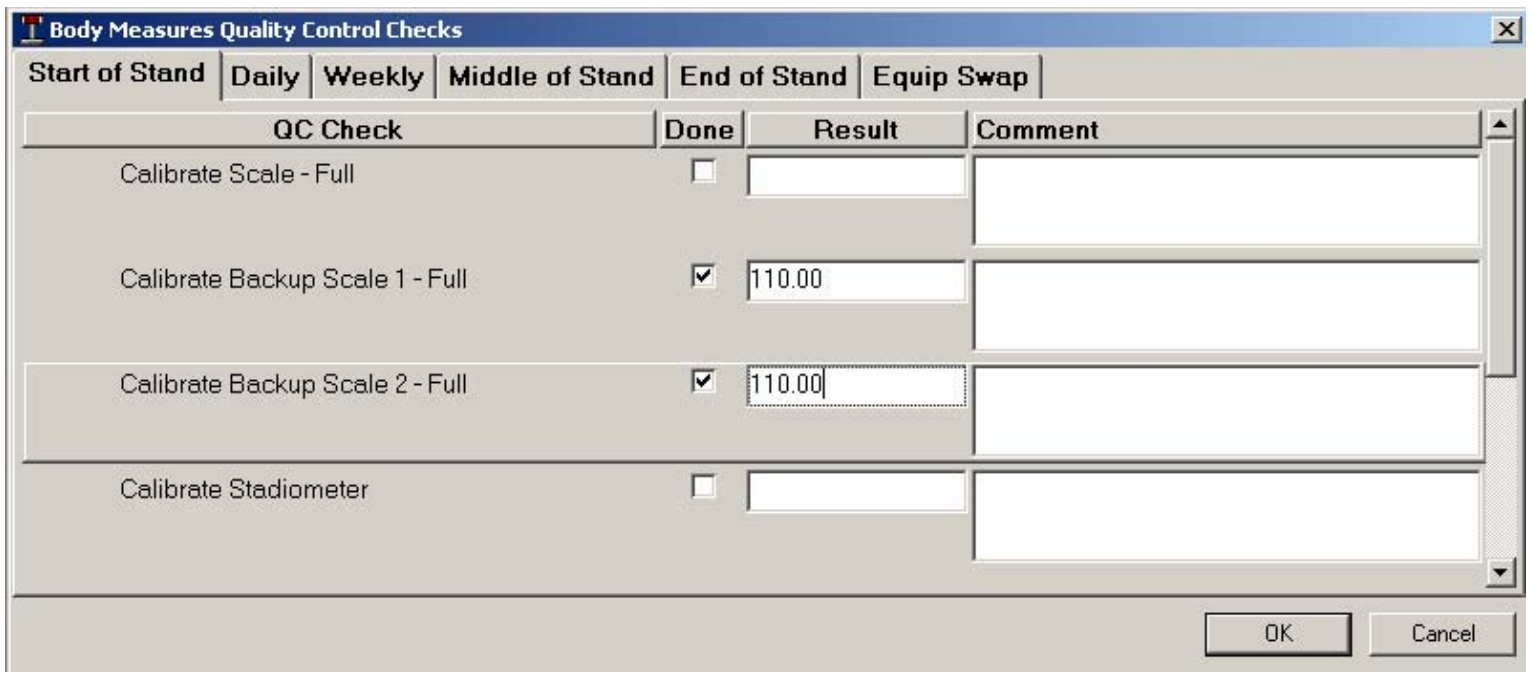


- The acceptable calibration weight range for 11, 10 kilogram calibration weights on the portable scale is $109.50-110.50 \mathrm{~kg}$.

- If the result falls outside the acceptable range, inform the chief technologist, MEC manager, and home office component specialist to have the scale replaced. Document the issue in the UFO system.

- Follow the above calibration procedures for the second portable scale, under "Calibrate Backup Scale 2 - Full."

- If either scale is replaced at a later date, record the calibration results under the Equipment Swap tab of the QC Checks dialog box.

\subsubsection{Stadiometer}

\section{Calibration: Start of Stand, Weekly}

- Insert one end of the $80 \mathrm{~cm}$ calibration rod in the circular indentation on the stadiometer base.

- Lower the stadiometer head piece to rest firmly against the top end of the calibration rod. Ensure that the rod stands perpendicular to the base.

- Check the result displayed on the digital measurement device. When calibrated, the result should read $80.0 \mathrm{~cm}$.

- Click on the appropriate tab (i.e., "Start of Stand" or "Weekly") in the ISIS QC Checks dialog box (Exhibit 5-10).

- Click the "Done" box beside "Calibrate Stadiometer" to capture the value directly into ISIS under the Result field.

- If the digital measurement device result was not $80.0 \mathrm{~cm}$, recalibrate the device by pressing the + or - button until the display reads $80.0 \mathrm{~cm}$. Record "Recalibrated to 80.0" in the Comment field (Exhibit 5-11). 
Exhibit 5-10. Stadiometer calibration: In calibration

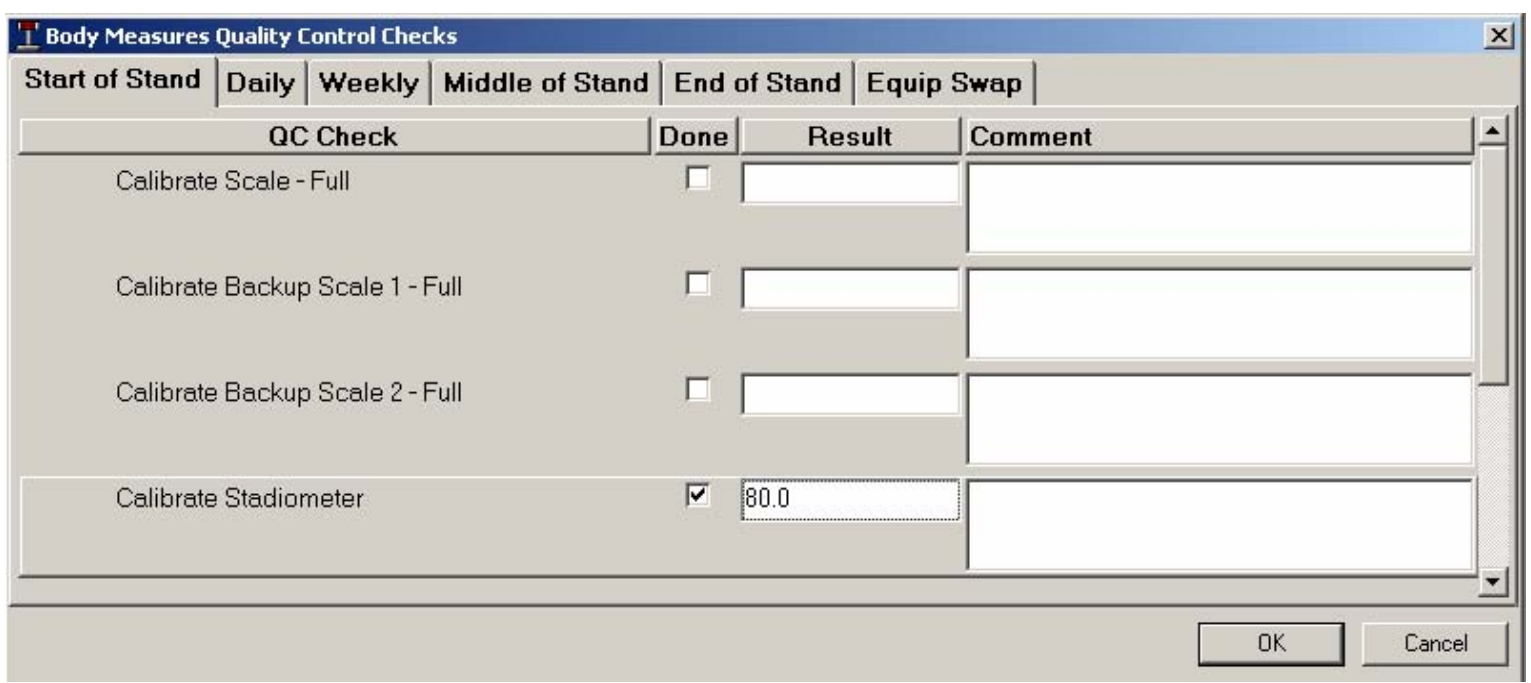

Exhibit 5-11. Stadiometer calibration: Out of calibration - recalibrated

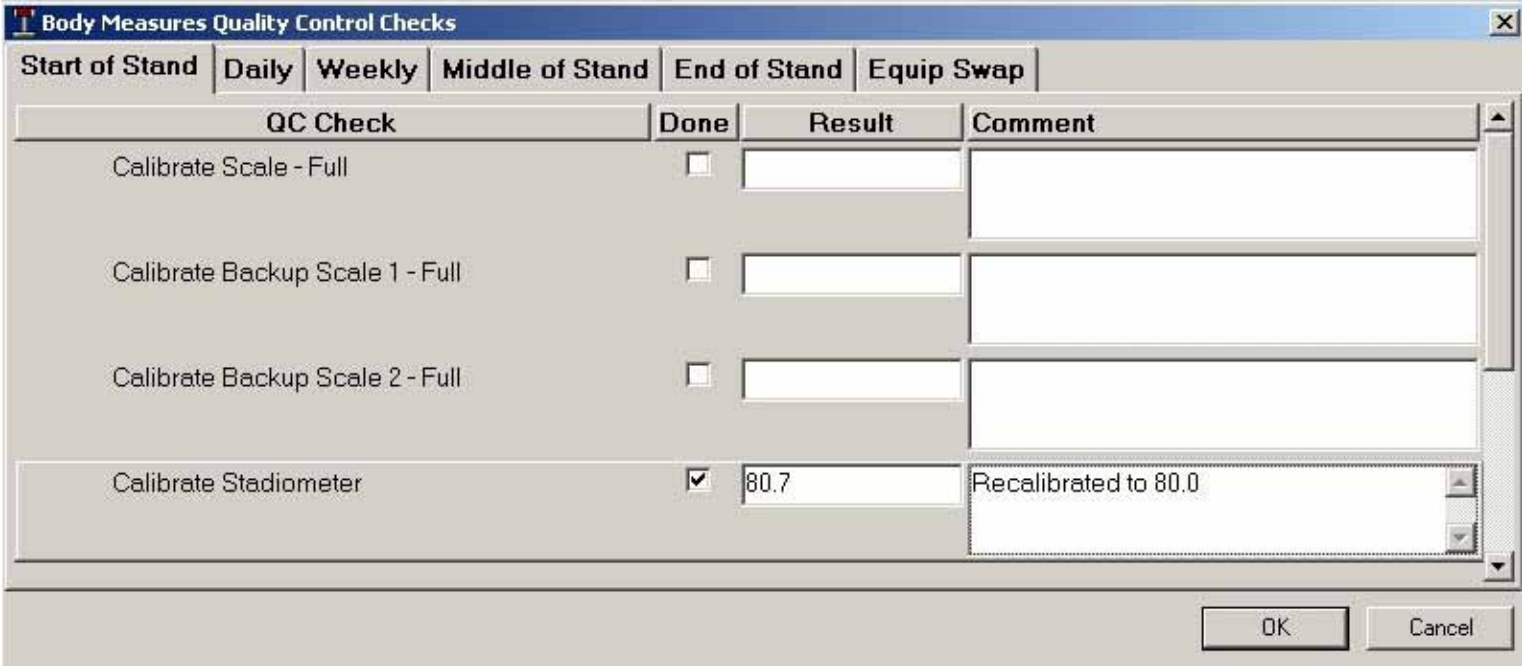




\subsubsection{Infantometer}

\section{Calibration: Start of Stand, Weekly}

- Slide the foot piece as far as it will go toward the head board of the infantometer.

- Check the result displayed on the digital measurement device.

When calibrated, the result should read as follows for each MEC:

- $\quad$ MEC 1: $16.0 \mathrm{~cm}$

- $\quad$ MEC 2: $0.0 \mathrm{~cm}$

- $\quad$ MEC 3: $0.0 \mathrm{~cm}$

- Click on the appropriate tab (i.e., "Start of Stand" or "Weekly") in the ISIS QC Checks dialog box (Exhibit 5-12).

- Click the "Done" box beside "Calibrate Infantometer" to capture the value directly into ISIS under the Result field.

- If the digital measurement device result was not $16.0 \mathrm{~cm}$ (MEC 1) or $0.0 \mathrm{~cm}$ (MEC 2 and 3), recalibrate the device by pressing the + or the - button until the display reads $16.0 \mathrm{~cm}$ (MEC 1) or $0.0 \mathrm{~cm}$ (MEC 2 and 3). Record "Recalibrated to [16.0 or 0.0 ]" in the Comment field (Exhibit 5-13). 
Exhibit 5-12. Infantometer calibration: In calibration

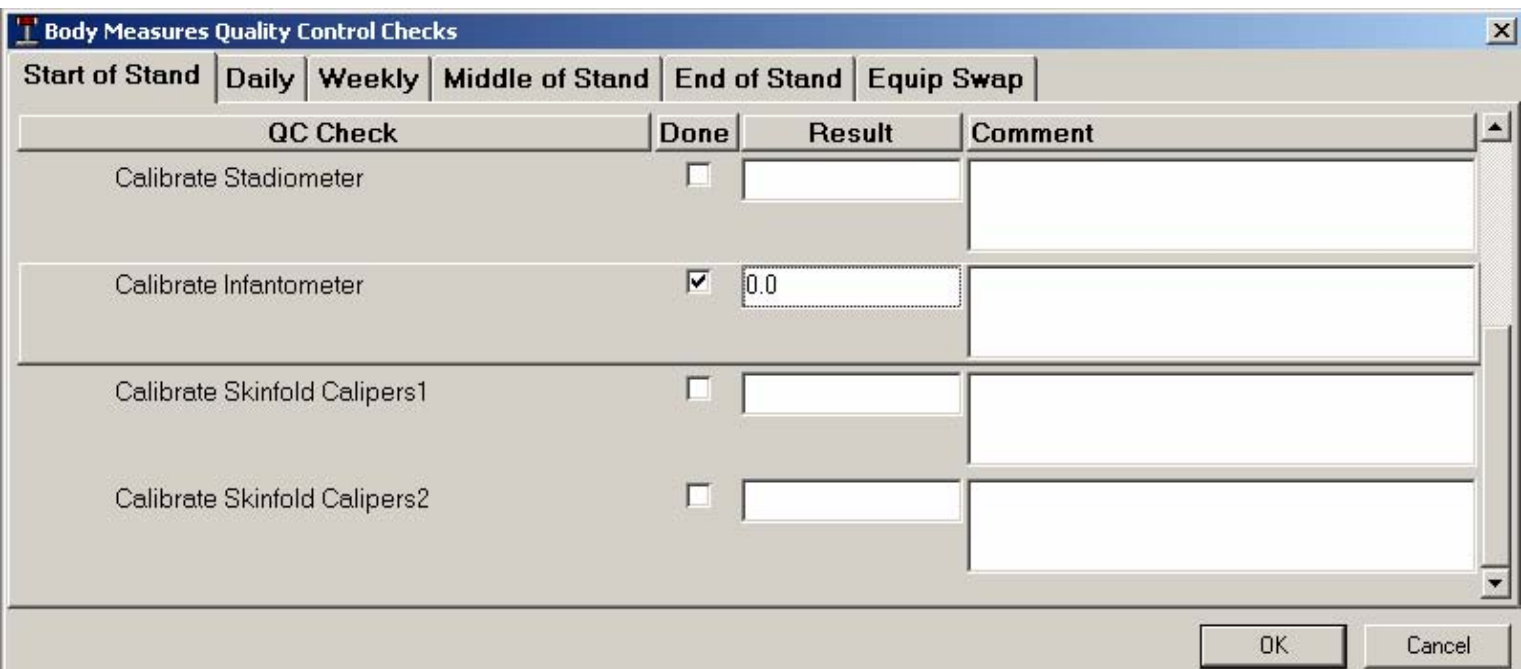

Exhibit 5-13. Infantometer calibration: Out of calibration - recalibrated

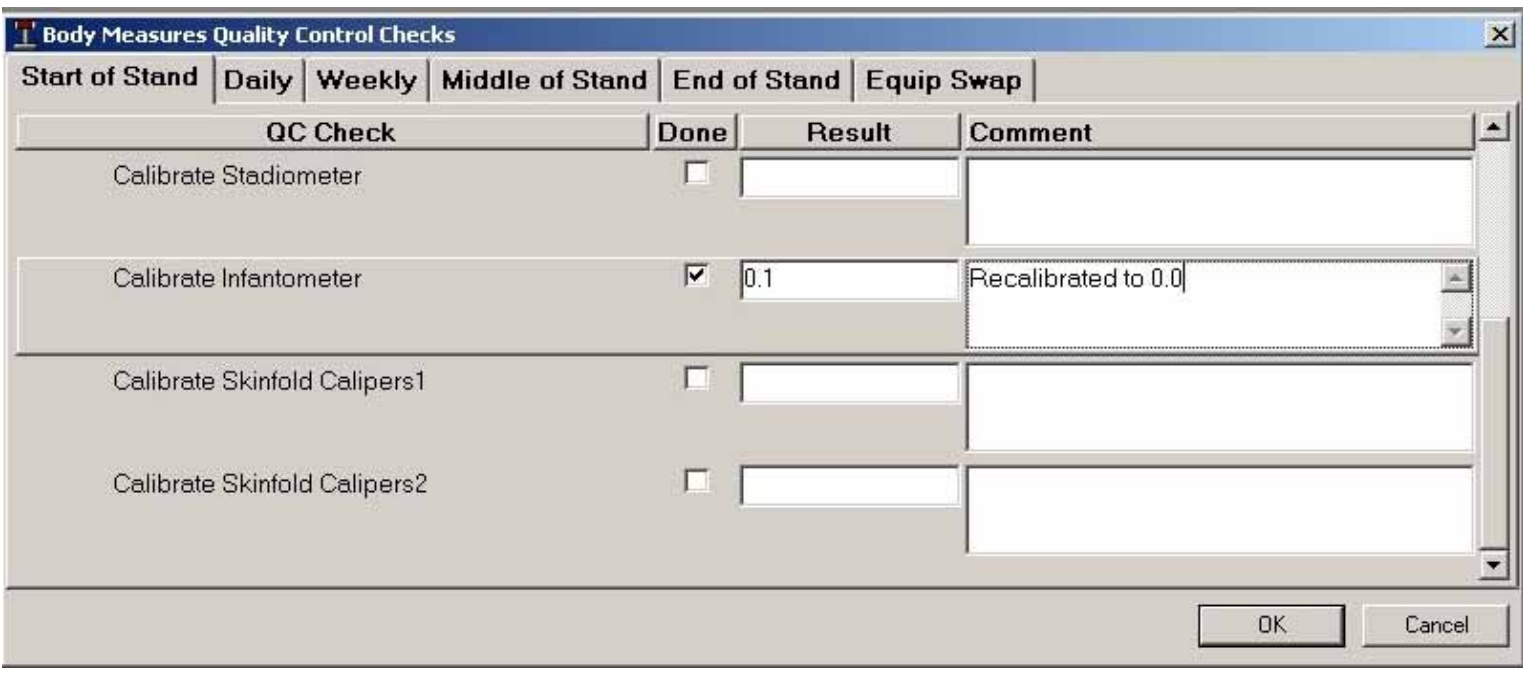




\subsubsection{Skinfold Calipers}

\section{Calibration: Start of Stand, Weekly}

- Click on the appropriate tab (i.e., "Start of Stand" or "Weekly") in the ISIS QC Checks dialog box (Exhibit 5-7).

- Click the "Done" box beside "Calibrate Skinfold Calipers 1."

- Record the caliper serial number in the Result field, for example, "60709."

- "Zero" the calipers:

- $\quad$ Check that the pointer falls exactly on $0 \mathrm{~mm}$.

- If the pointer is off, loosen the screw knob on top of the dial, turn the dial slowly until the pointer reads zero, and retighten the screw.

- Place the step wedge standard between the caliper jaws at each of the four steps.

Check that the reading falls within the following acceptable ranges:

- $\quad$ Step 1: $9.8-10.5 \mathrm{~mm}$

- $\quad$ Step 2: $19.8-20.5 \mathrm{~mm}$

- $\quad$ Step 3: $29.9-30.5 \mathrm{~mm}$

- $\quad$ Step 4: $39.8-40.4 \mathrm{~mm}$

- Record the measurement taken at all four steps in the Comment field, for example: “10.2, 20.4, 30.3, 39.9."

NOTE: The reason for entering the caliper results under Comments and the caliper serial number under Results is because the Result field has a standard character length of 15 characters, which does not accommodate the caliper results. 
Exhibit 5-14. Skinfold caliper calibration

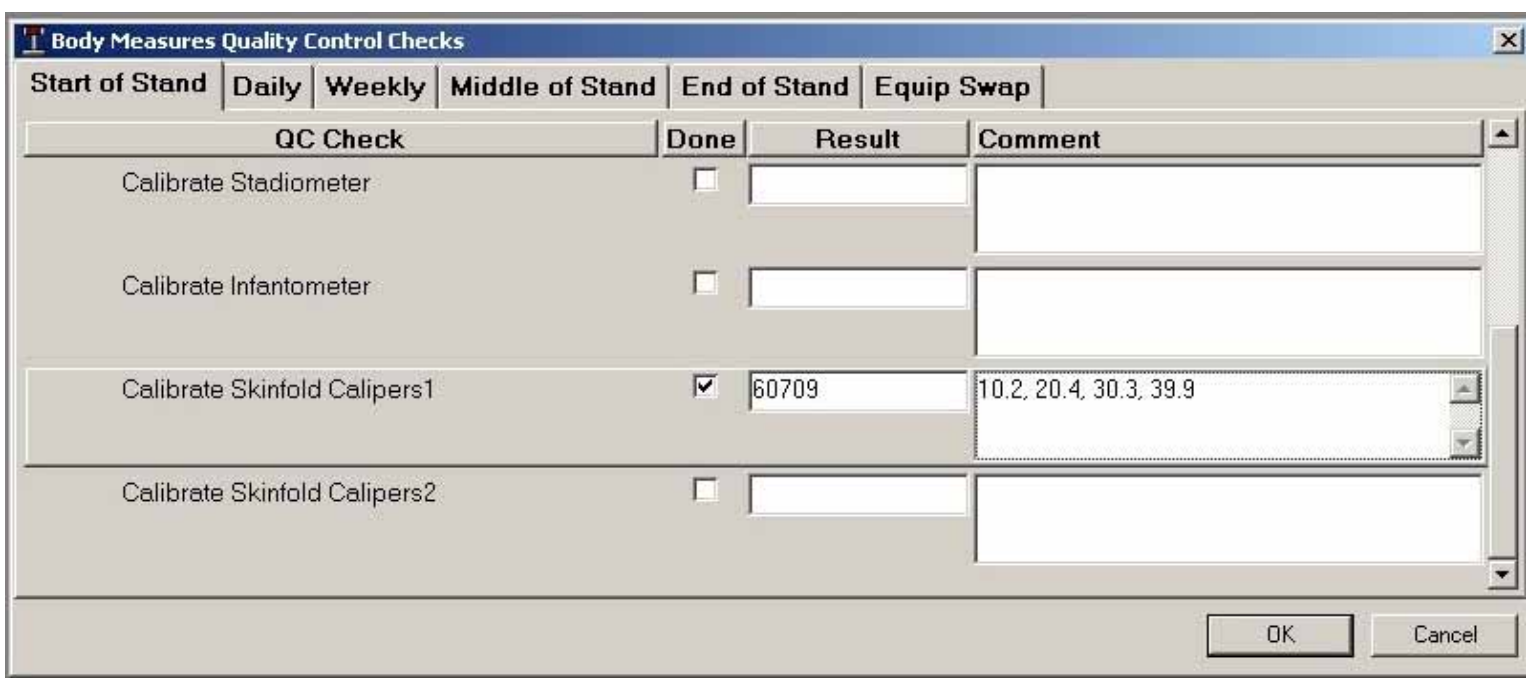

- If any readings fall outside the acceptable range, inform the chief tech, MEC manager, and home office component specialist to have the calipers replaced. Document the issue in the UFO system.

- Follow the above calibration procedures for the second set of skinfold calipers, under "Calibrate Skinfold Calipers 2."

- If the calipers are replaced at a later date, record the calibration results under the Equipment Swap tab of the QC Checks dialog box.

\subsubsection{Equipment Swap}

If any equipment requires recalibration or replacement outside of the regularly scheduled calibrations, i.e., Start of Stand, Daily, Weekly, or Middle of Stand, record the new calibrations under the Equipment Swap tab of the ISIS Body Measures Quality Control Checks screen.

- Click the "Equipment Swap" tab in the Quality Control Checks dialog box (Exhibit 5-15).

- Click the "Done" box beside the equipment piece that will be recalibrated or replaced. For example, in Exhibit 5-16 serial \#60705 belongs to the replacement/recalibrated skinfold calipers. 
- For each equipment piece(s) to be recalibrated or replaced, complete the Result and/or Comment field with the new calibration information in accordance with the calibration procedures described in Sections 5.2.1 to 5.2.6.

- When you are finished entering the new calibrations, click OK to exit the QC screen.

- ISIS will automatically save the equipment swap information permanently to the study database. However, similar to the daily QC process, when the tech opens a new session the Equipment Swap tab will appear blank, as shown in Exhibit 5-15. This means ISIS is ready for additional calibrations to be entered if necessary.

Exhibit 5-15. Equipment swap - blank

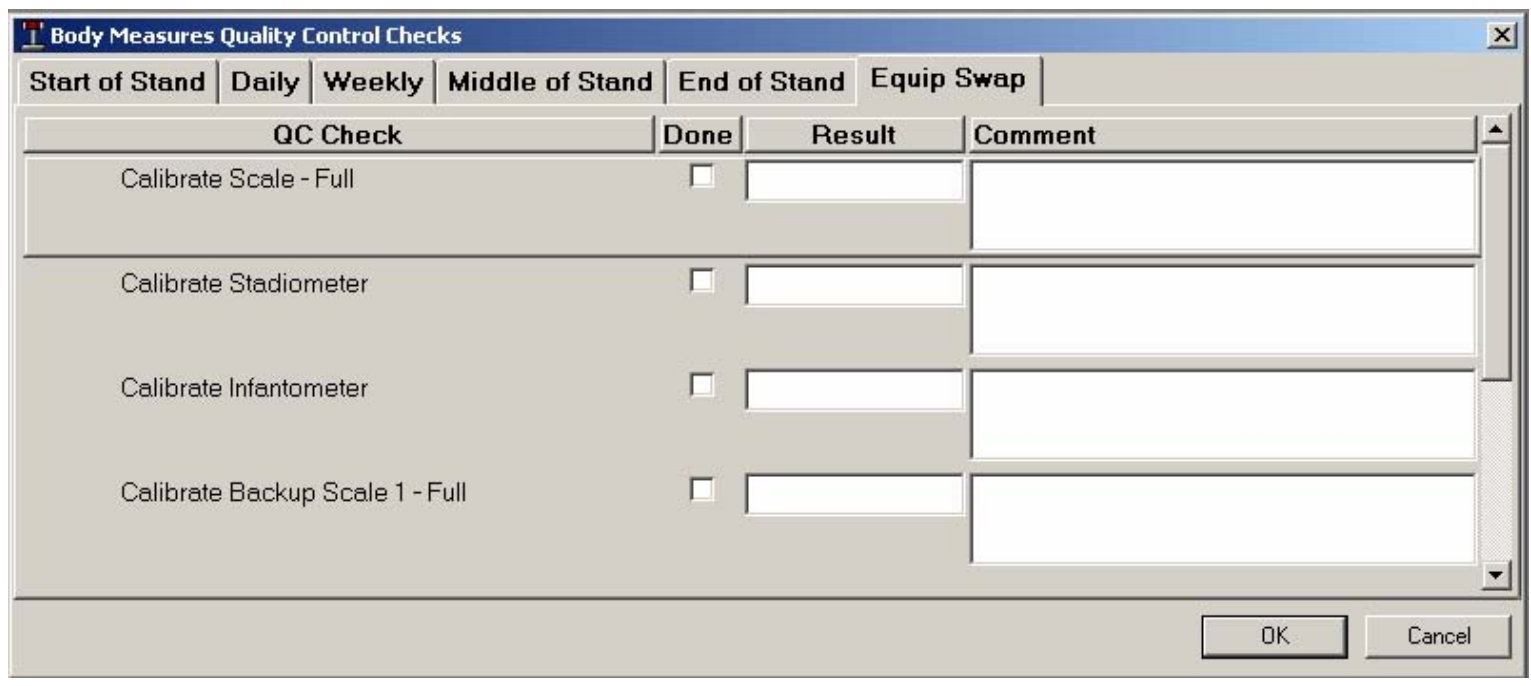

Exhibit 5-16. Equipment swap - skinfold caliper swapped

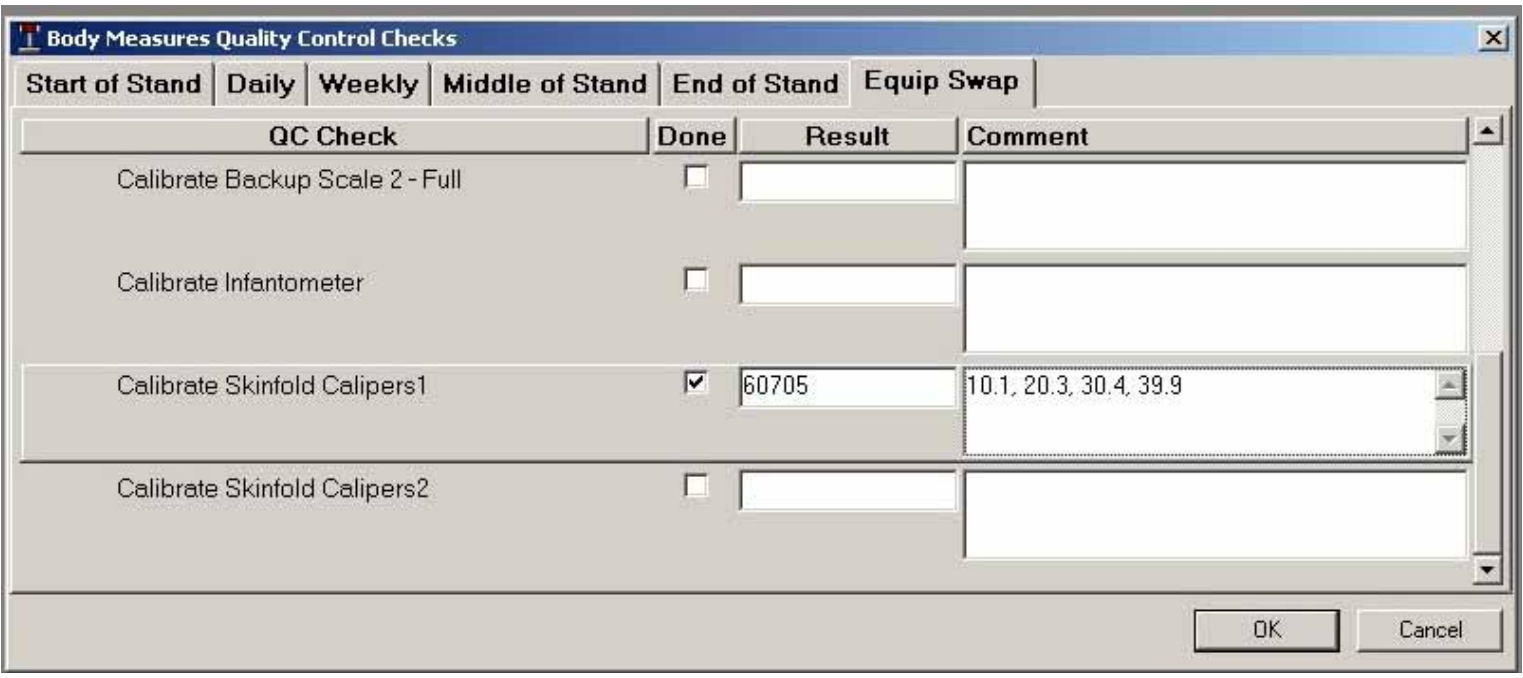




\subsection{ISIS Examination Screens}

The ISIS anthropometry examination screens are designed to be user friendly and at the same time assure the collection of accurate data. Quality control features of the ISIS anthropometry application include:

- The weight, length, and height measurement data is transmitted directly from the equipment into ISIS by clicking the "Get" button.

- The application will not permit advancement to the next screen unless a number or a comment is entered in every field.

- When the application advances to the next screen, the cursor will automatically appear in the appropriate field awaiting data entry.

- Each measurement number entered is verified using a computer-generated voice that repeats the measure aloud immediately following data capture.

- The risk of measurement and recording errors is reduced through the use of preprogrammed edit ranges. For example, if data captured in ISIS does not fall within the 1 st or 99th percentile based on the NHANES III dataset, an "out of range" message will appear on the screen. Although some data collected, i.e., from very small or very large SPs, may truly fall outside of the "normal" ranges, the examiner and recorder must verify the measurement if prompted by ISIS.

- Data entry errors are also minimized by limits set on the placement of decimal points and the number of digits entered. For instance, if the number entered for a measurement exceeds the allowable digits, ISIS will not permit the number to be entered.

\subsection{Observations, Replication, and Review}

A consulting anthropometry expert will visit each MEC team twice per year at minimum. These visits will serve to verify that the anthropometry protocol is being implemented properly and consistently. While at the MEC, the expert will meet with the technologists to review any problems or variations in the standard procedures. Following the site visit, the consultant will summarize all of the issues encountered during the visit in a final report to Westat. The Westat component specialist will forward a copy of the consultant's Site Visit Report to the appropriate NCHS project officer. 
Besides the observation of anthropometry examinations, a critical function of the body measures consultant is to serve as the "gold standard" anthropometry examiner. In this role, the consultant will conduct a repeat anthropometry examination on each SP immediately following the examination performed by the technologist. These gold standard replicate exams will be used to compare measurements between the expert consultant and the individual technologists. Gold standard examinations thus comprise an essential aspect of quality control for the anthropometry component. Appendix B explains the procedures for conducting anthropometry gold standard replicate examinations.

In addition to observations and gold standard exams completed by the anthropometry consultant, NCHS personnel and Westat component staff will visit the MEC teams at regular intervals to observe anthropometry examinations. To further monitor the quality of data collection, Westat component staff will generate reports from the ISIS intraweb. The number of anthropometry examinations and examination times: cumulative and sorted by session, by age group, and by technologist, as well as the reason for not done and partial examinations, will be analyzed for each stand.

Finally, retraining sessions will be arranged by the Westat component specialist in coordination with the NCHS project officer and the anthropometry consultant when major protocol changes are introduced, or when a lack of standardization is observed among the technologists. 


\section{SAFETY PROCEDURES}

\section{1 $\quad$ Equipment Precautions}

The safety of the survey participants and staff is paramount. All of the anthropometry equipment is cleaned, maintained, and calibrated regularly not only to maintain data quality but also to protect the equipment, SPs, and exam staff. If any equipment breaks or malfunctions, discontinue its use and notify the chief tech, MEC manager, and home office component specialist. Document all equipment issues in the UFO and ETS utilities as appropriate. As stated in Section 2.5, each MEC has select backup equipment available and the home office keeps a complete set of backup equipment. When necessary, the Westat component specialist will arrange for the replacement or repair of any malfunctioning equipment.

\subsection{Movement and Positioning}

The process of taking body measurements does not impose any physical harm or risk to survey participants. However, for safety reasons the technologist and recorder must observe certain precautions when positioning SPs for the required measurements. For example, participants who appear unsteady on their feet should be encouraged to grasp the metal safety bar affixed to the exam room wall during the taking of weight, circumference, and skinfold measurements. In particular, performing body measurements on children requires additional safety precautions and constant supervision by the exam staff. For instance, the technologist and recorder must hold children carefully on the infantometer to prevent any falls, as infants and young children tend to flip themselves over very quickly. Also, small children aged 2-6 years will be placed standing on the sitting box so that the technologist can control their movements and take measurements at eye level. The box is situated by the mirror to allow the child to grab the metal safety bar on the wall for balance. Ensure that all anthropometric equipment and supplies are always out of reach. Most importantly, NEVER leave a child unattended.

As described in Section 3.3.1.12, only a subset of anthropometric measurements will be attempted on participants in wheelchairs. If the SPs can position themselves toward the right side of the wheelchair and their shirt allows access to the right arm, the technologist should collect the upper arm length, arm circumference, and triceps skinfold measurements. In the case of amputees (Section 3.3.1.13), the technologist should obtain body measurements on the left side when any part of the right limb is amputated. 


\subsection{Emergency Procedures}

For any emergency or otherwise safety-related situations concerning SPs during the anthropometry exam, the technologist should summon the MEC manager and physician immediately. The NHANES mobile examination center has well-developed emergency response protocols which will be implemented in the event of any emergency. Refer to the NHANES Safety Issues and Emergency Procedures Manual for complete details. 
Appendix A

Body Measures Recording Form 


\section{Body Measures Recording Form}

SP ID:

Technologist:
Stand \#:

Session/Date:

Recorder:

\begin{tabular}{|c|c|c|c|c|c|c|c|}
\hline \multicolumn{2}{|c|}{ MEASURES } & BIRTH + & $2 \mathrm{MO}+$ & $7 \mathrm{MO}+$ & 2YR+ & 4YR+ & 8YR+ \\
\hline (BMXWT) & Weight & & & & & & \\
\hline (BMXRECUM) & Recum Length & & & & & & \\
\hline (BMXHEAD) & Head Circ & & & & & & \\
\hline (BMXHT) & Height & & & & & & \\
\hline (BMXHTCRA) & $\begin{array}{l}\text { Ht Correction: } \\
\text { Above Waist }\end{array}$ & & & & & & \\
\hline (BMXHTCRB) & $\begin{array}{l}\text { Ht Correction: } \\
\text { Below Waist }\end{array}$ & & & & & & \\
\hline (BMXLEG) & Leg Length & & & & & & \\
\hline (BMXARML) & Upper Arm Length & & & & & & \\
\hline \multicolumn{8}{|c|}{ Mid-point of Upper Arm Length } \\
\hline (BMXARMC) & Arm Circ & & & & & & \\
\hline (BMXWAIST) & Waist Circ & & & & & & \\
\hline (BMXTRI) & Triceps SF & & & & & & \\
\hline (BMXSUB) & Subscap SF & & & & & & \\
\hline \multicolumn{8}{|c|}{ (BMXAMP) Amputations? $\quad$ Yes/No } \\
\hline If Yes: & & & & & & & \\
\hline $\begin{array}{l}\text { (BMXUREXT) } \\
\text { Upper R Extremity? }\end{array}$ & Yes/No & & & & & & \\
\hline $\begin{array}{l}\text { (BMXUPREL) } \\
\text { Upper R. Above/Be }\end{array}$ & low Elbow? & & & & & & \\
\hline $\begin{array}{l}\text { (BMXULEXT) } \\
\text { Upper L Extremity? }\end{array}$ & Yes/No & & & & & & \\
\hline $\begin{array}{l}\text { (BMXUPLEL) } \\
\text { Upper L Above/Belc }\end{array}$ & ow Elbow? & & & & & & \\
\hline $\begin{array}{l}\text { (BMXLOREX) } \\
\text { Lower R Extremity? }\end{array}$ & Yes/No & & & & & & \\
\hline $\begin{array}{l}\text { (BMXLORKN) } \\
\text { Lower R. Above/Bel }\end{array}$ & low Knee? & & & & & & \\
\hline $\begin{array}{l}\text { (BMXLLEXT) } \\
\text { Lower L Extremity? }\end{array}$ & Yes/No & & & & & & \\
\hline $\begin{array}{l}\text { (BMXLLKNE) } \\
\text { Lower L. Above/Bel }\end{array}$ & low Knee? & & & & & & \\
\hline
\end{tabular}


Appendix B

Procedures for Conducting Anthropometry Gold Standard Replicate Examinations 


\section{PROCEDURES FOR CONDUCTING ANTHROPOMETRY GOLD STANDARD REPLICATE EXAMINATIONS}

Gold standard examinations are an essential quality control measure for the anthropometry component. These replicate exams are used to compare differences in measurement results obtained by the expert consultant to those obtained by the individual technologists. Below are instructions for conducting gold standard replicate examinations at the MEC.

At the start of the session during which gold standard replicate exams will be conducted, activate the Gold Standard software application:

- In the ISIS Anthropometry application, under the Utilities menu, go to "Gold Standard."

- Click on "Gold Standard" so that a check mark appears beside it, indicating the application is on.

- If rebooting the computer becomes necessary during a session, the Gold Standard application must be reactivated.

To use the Gold Standard application, the technologists should conduct exams as usual, with

one person examining and a second person recording. Following the primary examination, the Gold Standard recruitment screen will appear with two questions:

1. Do you want to conduct a gold standard examination on this SP?

2. Does the SP consent to a gold standard examination?

If a gold standard examination will not be conducted at this time, answer "No" to the first question; the second question will remain deactivated. If "Yes" is answered to the first question, ISIS will activate the second question. In order to repeat the examination, verbal consent must then be obtained from the SP:

"In order to assure the high quality of our data we sometimes repeat the exam. Would you mind if we repeat the exam?"

If the SP does not give consent, or if for some other reason a gold standard exam will not be conducted at this time, simply advance to the next screen, the status screen, and end the gold standard 
exam. If the SP gives consent, enter "Yes" to the second recruitment question and select the appropriate name under the Gold Standard Examiner and Gold Standard Recorder drop-downs.

For the gold standard exam, the anthropometry consultant will serve as the examiner and the original examiner should record for the gold standard exam. If this is not possible, the recorder from the primary exam can continue to record for the gold standard exam. In preparation for the gold standard exam, the cosmetic pencil marks should be left on the SP for the consultant's reference.

In ISIS the gold standard application screens are identical to the primary exam screens, except that the SP information screen and amputation questions will not appear. Also, the status of gold standard exams will always default to "Complete" regardless of whether the actual status of this section was partial or not done. This is designed to prevent the status of the gold standard section from affecting the overall status of the component.

Ensuring that SPs complete their primary exams at the MEC remains a top priority. If conducting gold standard replicate exams will impede the flow of SPs through the MEC, the anthropometry consultant can instead observe the primary exams or act as the recorder. By freeing up other staff, this may allow replicate exams to resume. Communication among all members of the MEC team will promote a smooth work flow to this end. 